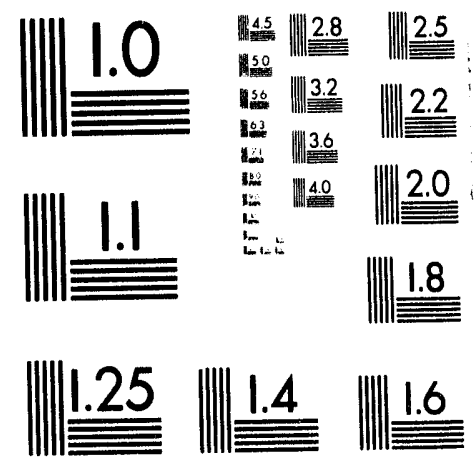



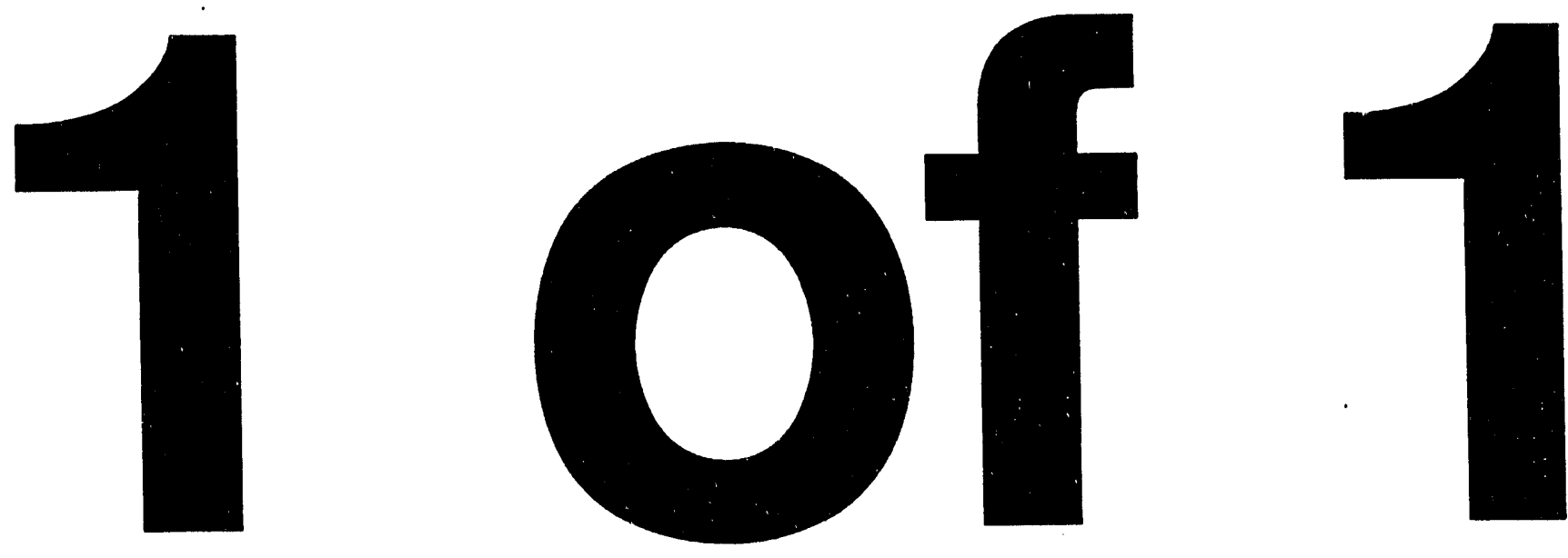
ES/ER/TM-96

\section{Toxicological Benchmarks for Screening of Potential Contaminants of Concern for Effects on Aquatic Biota on the Oak Ridge Reservation, Oak Ridge, Tennessee}

G. W. Suter II

M. A. Futrell

G. A. Kerchner

RECEIVED

FEB 091994

OSTI 
ES/ER/TM-96

Energy Systems Environmental Restoration Program

ORNL Environmental Restoration Program

Toxicological Benchmarks for Screening of Potential Contaminants of Concern for Effects on Aquatic Biota on the Oak Ridge Reservation, Oak Ridge, Tennessee

\author{
G. W. Suter II \\ M. A. Futrell \\ G. A. Kerchner
}

Date Issued-September 1992

Prepared by

Environmental Sciences Division

Oak Ridge National Laboratory

ESD Publication 3940

Prepared for

U.S. Department of Energy

Office of Environmental Restoration and Waste Management under budget and reporting code EW 20

OAK RIDGE NATIONAL LABORATORY

Oak Ridge, Tennessee 37831-6285

managed by

MARTIN MARIETTA ENERGY SYSTEMS, INC.

for the

U.S. DEPARTMENT OF ENERGY

under contract DE-AC05-84OR21400 


\section{Author Affiliations}

G. A. Suter II is a member of the Environmental Sciences Division, Oak Ridge National Laboratory, Martin Marietta Energy Systems, Inc. M. A. Futrell is a student at East Tennessee State University, and G. A. Kerchner is a student at Harvard College.

\section{DISCLAIMER}

This report was prepared as an account of work sponsored by an agency of the United States Government. Neither the United States Government nor any agency thereof, nor any of their employees, makes any warranty, express or implied, or assumes any legal liability or responsibility for the accuracy, completeness, or usefulness of any information, apparatus, product, or process disclosed, or represents that its use would not infringe privately owned rights. Reference herein to any specific commercial product, process, or service by trade name, trademark, manufacturer, or otherwise does not necessarily constitute or imply its endorsement, recommendation, or favoring by the United States Government or any agency thereof. The views and opinions of authors expressed herein do not necessarily state or reflect those of the United States Government or any agency thereof. 


\section{CONTENTS}

TABLES $\ldots \ldots \ldots \ldots \ldots \ldots \ldots \ldots \ldots \ldots \ldots \ldots \ldots \ldots \ldots \ldots \ldots \ldots \ldots$

ACKNOWLEDGEMENTS $\ldots \ldots \ldots \ldots \ldots \ldots \ldots \ldots \ldots \ldots \ldots \ldots \ldots \ldots$ vii

EXECUTTVE SUMMARY $\ldots \ldots \ldots \ldots \ldots \ldots \ldots \ldots \ldots \ldots \ldots \ldots \ldots \ldots$

1. INTRODUCTICN $\ldots \ldots \ldots \ldots \ldots \ldots \ldots \ldots \ldots \ldots \ldots \ldots \ldots \ldots \ldots$

2. METHODS FOR DERIVING BENCHMARKS $\ldots \ldots \ldots \ldots \ldots \ldots \ldots \ldots$

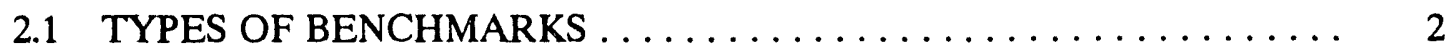

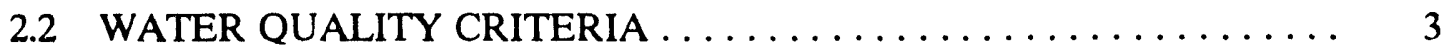

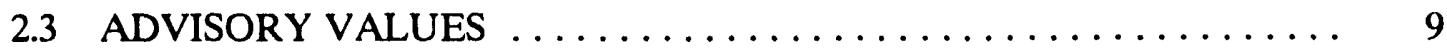

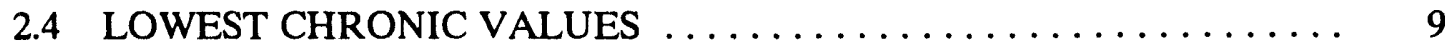

2.5 ESTIMATED LOWEST CHRONIC VALUES .............. 9

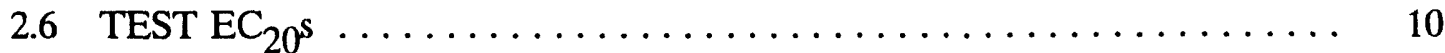

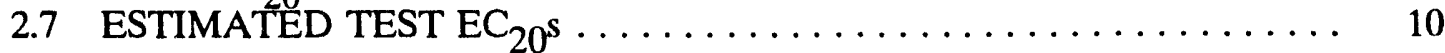

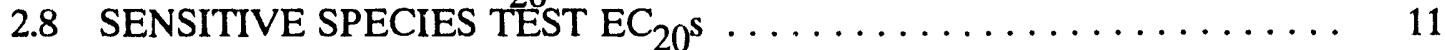

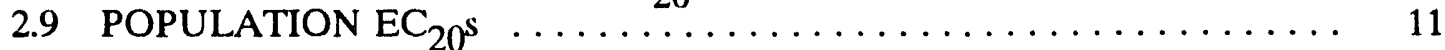

2.10 BACKGROUND CONCENTRATIONS $\ldots \ldots \ldots \ldots \ldots \ldots \ldots \ldots \ldots$

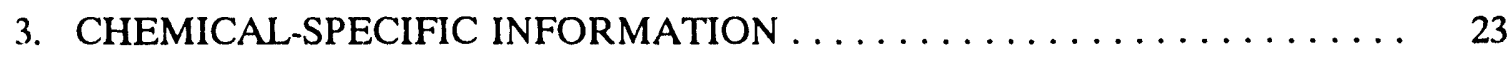

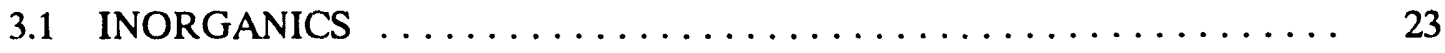

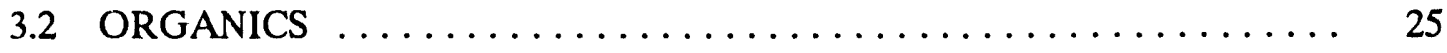

4. COMPARISON OF BENCHMIARKS $\ldots \ldots \ldots \ldots \ldots \ldots \ldots \ldots \ldots \ldots$

5. APPLICATION OF BENCHMARKS $\ldots \ldots \ldots \ldots \ldots \ldots \ldots \ldots \ldots \ldots$

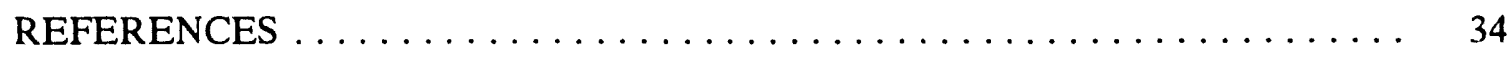

Appendix A-DATA USED FOR ADVISORY VALUE CALCULATIONS . . . . A A-1

Appendix B-FACTORS FOR ESTIMATION OF THE PESTICIDE ACUTE

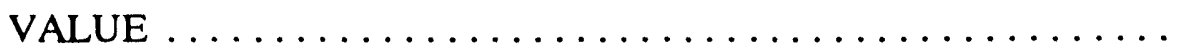




\section{TABLES}

1 Summary of benchmarks for priority contaminants in fresh water $\ldots \ldots \ldots \ldots$

2 Concentrations estimated to cause a $20 \%$ reduction in the recruit abundance of largemouth bass, with upper and lower

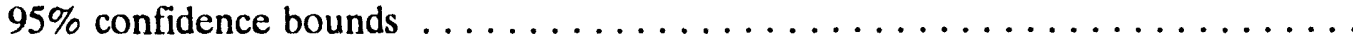

3 Comparisons of alternative screening benchmarks for aquatic life on the basis of the number of chemicals for which each could be calculated $(n)$, the percentage of those chemicals for which it was the lowest benchmark, and the median, minimum, and maximum ratio of each benchmark to the chronic NAWQC for

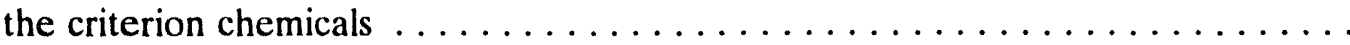

4 Amount of data available and used to calculate advisory values . . . . . . . . 29

5 Quotients of ratios of NAWQC/advisory values calculated for those chemicals for which NAWQC were available 


\section{ACKNOWLEDGMENTS}

The manuscript benefitted from the review comments of Gordon Blaylock, Richard Bonczek, Ruth Hull, Lynn Kszos, Joni Skiles, and Mark Stack. 


\section{EXECUTIVE SUMMARY}

One of the initial stages in ecological risk assessment of hazardous waste sites is the screening of contaminants to determine which of them are worthy of further consideration. This process is termed "contaminant screening." Screening is performed by comparing concentrations in ambient media to benchmark concentrations that are either indicative of a high likelihood of significant effects (upper screening benchmarks) or of a very low likelihood of significant effects (lower screening benchmarks). Exceeding an upper screening benchmark indicates that the chemical in question is clearly of concern and remedial actions are likely to be needed. Exceeding a lower screening benchmark indicates that a contaminant is of concern unless other information indicates that the data are unreliable or the comparison is inappropriate. Chemicals with concentrations below the lower benchmark are not of concern if the ambient data are judged to be adequate.

This report presents potential screening benchmarks for protection of aquatic life from contaminants in water. Because there is no guidance for screening benchmarks, a set of alternative benchmarks is presented here. The alternative benchmarks are based on different conceptual approaches to estimating concentrations causing significant effects. For the upper screening benchmark, they are the acute National Ambient Water Quality Criterion (NAWQC) and the acute pesticide advisory value (a concentration that is estimated with $95 \%$ confidence not to exceed the unknown acute NAWQC for those chemicals with no NAWQC). The alternative chronic benchmarks are the chronic NAWQC, the chronic pesticide screening value, the lowest chronic values for fish and daphnids, the lowest concentration that produces a response in $20 \%$ of fish and daphnids [effective concentration $20\left(\mathrm{EC}_{20}\right)$ ] as established through chronic toxicity tests, the estimated $\mathrm{EC}_{20}$ for a sensitive species, and the concentration estimated to cause a $20 \%$ reduction in the recruit abundance of largemouth bass. It is recommended that ambient chemical concentrations be compared to all of these benchmarks. If NAWQC are exceeded, the chemicals must be contaminants of concern because the NAWQC are applicable and relevant or appropriate requirements. If NAWQC are not exceeded, but other benchmarks are, contaminants should be selected on the basis of the number of benchmarks exceeded and the conservatism of the particular benchmark values, as discussed in this report.

To the extent that toxicity data are available, this report presents the alternative benchmarks for chemicals that have been detected on the Oak Ridge Reservation. It also presents the data used to calculate the benchmarks, and the sources of the data. It compares the benchmarks and discusses their relative conservatism and utility. 


\section{INTRODUCTION}

An important early step in the assessment of ecological risks posed by a contaminated site is the screening of contaminants. In most cases, concentrations in water will be reported for over 100 chemicals, many of which will be reported as undetected at some defined limit of detection. The assessor must decide which of the detected chemicals constitute an ecological hazard and which of the undetected chemicals may pose a hazard at concentrations below the reported detection limits. This screening is done by comparing the reported concentrations to toxicological benchmarks. If concentrations of a chemical exceed its benchmark for a particular medium, then it is worthy of further measurement and assessment. If not, it can be ignored (assuming that the analytical data are adequate).

In practice, a series of benchmarks of differing conservatism may be used. Exceedance of an upper screening benchmark would suggest a severe hazard and the need for urgent action. Nonexceedance of a lower screening benchmark would suggest that no hazard exists. Exceedance of an increasing number of benchmarks would constitute increasing evidence of the need for measurement and assessment.

The purpose of this report is to present and analyze alternate toxicological benchmarks for screening chemicals for aquatic ecological effects. Screening benchmarks have not been previously proposed, and no guidance for developing such values is available from the U.S. Environmental Protection Agency (EPA) or other regulatory agencies. In general, the National Ambient Water Quality Criteria for Protection of Aquatic Life (NAWQC) have been used for this purpose. However, they were not designed for contaminant screening. They are intended to protect most aquatic species most of the time with reasonable confidence. Because screening benchmarks are intended to provide a high degree of confidence that a chemical is not hazardous, greater conservatism may be warranted. In addition, the NAWQC do not correspond to any particular type or level of effect (Suter et al. 1987). Because screening assessments are performed as the preliminary stage of ecological risk assessments, it is appropriate to consider potential toxicological benchmarks that correspond to defined types and levels of effects.

This compilation is limited to chemicals that have been detected on the Oak Ridge Reservation (ORR) and to benchmarks derived from studies of toxic effects on fresh water organisms. The list of chemicals detected on the ORR includes 45 metals and 105 industrial chemicals. Only four pesticides (chlordane, DDT, heptachlor, and lindane) occur on the list, and those are persistent and widespread. 


\section{METHODS FOR DERIVING BENCHMARKS}

\subsection{TYPES OF BENCHMARKS}

The simplest screening benchmarks are toxicity test endpoints. A test endpoint is a statistically derived numeric summary of the results of a toxicity test. Test endpoints can be calculated in two ways. First, a level of effect can be estimated by fitting a function such as the probit or logit to the concentration-response data to derive a concentration-response model. Then, by inverse regression, a concentration can be estimated that causes a particular level of effect such as the median lethal concentration $\left(\mathrm{LC}_{50}\right)$. Second, hypothesis-testing statistics can be used to determine whether each of the tested concentrations caused an effect that was statistically significantly different from the controls. The lowest concentration causing such an effect is termed the "lowest observed effect concentration" (LOEC); the highest concentration for which there were no such effects is termed the "no observed effect concentration" (NOEC). The geometric mean of the LOEC and NOEC is termed the "chronic value" (CV), and was formerly termed the "maximum acceptable toxicant concentration" (MATC).

Toxicity tests are conventionally divided into acute and chronic tests. Standard acute aquatic toxicity tests are $48 \mathrm{~h}$ or $96 \mathrm{~h}$ in duration and use juvenile or adult organisms; the test endpoints are the median lethal concentration $\left(\mathrm{LC}_{50}\right)$ or median effective concentration $\left(\mathrm{EC}_{50}\right)$ for death or some equivalent effect (e.g., immobilization). Standard chronic tests include all or most of the life cycle of the test organisms, and they include observations of growth, deformities, and reproductive success as well as lethality. The standard endpoint for chronic tests is the CV.

Another important distinction is between response-specific and integrative endpoints. Conventionally, NOECs and LOECs are calculated for each response parameter, and the results for the most statistically sensitive parameter are reported. Because effects on populations and ecosystems are a result of the integrated effects of the toxicant on all life stages, it makes more sense to integrate the responses in the test when calculating the test endpoint. Integrative endpoints may be simple arithmetic combinations of effects such as the proportional mortality across all tested life stages or population parameters derived from simple models such as the intrinsic rate of natural increase, $r$.

Benchmarks may be combinations of multiple test endpoints. An example is the chronic NAWQC which are derived from at least eight $\mathrm{LC}_{50}$ s and three CVs.

Finally, benchmarks may be derived by using mathematical models to simulate an assessment endpoint, a specific environmental characteristic that is valued and is at risk due to the contamination or disturbance that is being assessed (Suter 1989). For example, in this study we present concentrations estimated to correspond to a $20 \%$ reduction in recruit abundance for largemouth bass (Micropterus salmoides) because production of fish, particularly game tish, is an assessment endpoint for ORR ecological risk assessments (Suter et al. 1992). 


\subsection{WATER QUALITY CRITERIA}

The NAWQC are ARARs (applicable or relevant and appropriate requirements); therefore, they provide the basis for the screening benchmarks for contaminants in water. The acute NAWQC are calculated by the EPA as half the final acute value (FAV), which is the fifth percentile of the distribution of 48 - to $96-\mathrm{h} \mathrm{LC}_{50}$ values or equivalent median effective concentration $\mathrm{EC}_{50}$ values for each criterion chemical (Stephan et al. 1985). The acute NAWQC are intended to correspond to concentrations that would cause less than $50 \%$ mortality in $5 \%$ of exposed populations in a brief exposure. They may be used as a reasonable upper screening benchmark because waste-site assessments are concerned with sublethal effects and largely with continuous exposures rather than the lethal effects and episodic exposures to which the acute NAWQC is applied. The chronic NAWQC are the FAVs divided by the final acute-chronic ratio (FACR), which is the geometric mean of quotients of at least three $\mathrm{LC}_{50} / \mathrm{CV}$ ratios from tests of different families of aquatic organisms (Stephan et al. 1985). It is intended to prevent significant toxic effects in chronic exposures and is used in this assessment as one possible lower screening benchmark.

NAWQC for several metals are functions of water hardness; the criteria are lower for lower hardness levels. We used the criteria for $100 \mathrm{mg} / \mathrm{L}$ hardness that are reported by the EPA. That hardness is near the lower end of the range of hardness values reported for the Oak Ridge Reservation, so it is moderately conservative. For sites with different water hardnesses, site-specific criteria should be calculated. The NAWQC are listed in Table 1.

Many readers will note that the EPA's compilations of NAWQC contain values for many chemicals that have no NAWQC listed here (EPA 1986). That is because the EPA reports lowest CVs for those chemicals for which there is not enough data to calculate a criterion but for which there is at least one chronic value. Lowest CVs are treated as a separate category of benchmarks in this compilation. However, if sufficient data were available to calculate a final acute value (FAV), and if some chronic values were available but not the three or more needed to calculate a chronic criterion, the chronic criterion was approximated using the FAV and the geometric mean of the available chronic values. Those approximated criteria are noted in Table 1.

For particular chemicals, the lower screening benchmark could be lower than the chronic NAWQC for any one of the following five reasons. First, the chronic NAWQC are based on a threshold for statistical significance rather than biological significance. In some chronic tests, because of highly variable results, the statistical threshold corresponds to greater than $50 \%$ effect on a response parameter (Stephan and Rogers 1985, Suter et al. 1987). Second, not all important responses are included in the subchronic toxicity tests that are used to calculate many chronic NAWQC. In particular, effects on fecundity, which is the most sensitive response parameter on average in fish toxicity tests (Suter et al. 1987), are often not included. Third, the chronic NAWQC are based on the most statistically sensitive of the measured response parameters in each chronic or subchronic test. Therefore, cumulative effects over the life cycle of fish and invertebrates are not considered. Fourth, the NAWQC are set at a level that protects "most species most of the time." A lower screening benchmark should protect all species nearly all of the time. Finally, many of the NAWQC have not been revised since 1980 , so they do not incorporate recent data that are included in the calculation of other benchmarks. These concerns are confirmed by the recent finding that nickel concentrations on the ORR that are below chronic NAWQC nonetheless are toxic to daphnids (Kszos et al. 1992). 
Table 1. Summary of benchmarts for priority contaminants in fresh water (all values in micrograms per liter)

\begin{tabular}{|c|c|c|c|c|c|c|c|c|}
\hline \multirow[t]{2}{*}{ Chemical } & \multicolumn{2}{|c|}{$\begin{array}{l}\text { NAWQ criteria } \\
\text { advisory values }\end{array}$} & \multicolumn{2}{|c|}{$\begin{array}{l}\text { Lowest chronic } \\
\text { value for. }\end{array}$} & \multicolumn{2}{|c|}{$\begin{array}{l}\text { Lowest test } \mathrm{EC}_{20} \\
\text { value for: }\end{array}$} & \multirow{2}{*}{$\begin{array}{l}\text { SS test } \\
\mathrm{EC}_{20} \\
\end{array}$} & \multirow{2}{*}{$\begin{array}{c}\text { Population } \\
\mathrm{EC}_{20} \\
\end{array}$} \\
\hline & Acute & Chronic & Fish & Daphnids & Fish & Daphnids & & \\
\hline \multicolumn{9}{|c|}{ Inorganics } \\
\hline Aluminum & 750 & 87 & 5800 & 1900 & 4700 & 540 & 75 & - \\
\hline Antimony & $* 220$ & $* 18$ & 1600 & 5400 & 2310 & 1900 & - & 79 \\
\hline Arsenic III & 360 & 190 & 2962 & 914.1 & 2130 & 633 & 55 & 1995 \\
\hline Arsenic V & $* 27$ & •0.77 & 891.6 & 4450 & 1500 & $>932$ & - & 185 \\
\hline Barium & $* 4400$ & $* 109$ & - & $\cdot 20,336$ & - & - & - & - \\
\hline Beryllium & .55 & ${ }^{*} 0.61$ & $* 57$ & 5.3 & $* 148$ & 3.8 & - & 21 \\
\hline Boron & - & - & - & - & - & 7 & - & - \\
\hline Cadmium & 3.9 & 1.1 & 1.7 & 0.15 & 1.8 & 0.75 & $0.013^{\mathrm{a}}$ & 4.3 \\
\hline Calcium & - & - & - & - & - & - & - & - \\
\hline Chromium III & 1700 & 210 & 68.83 & $<44$ & 89 & - & 8.44 & 126 \\
\hline Chromium VI & 16 & 11 & 73.18 & 6.132 & 51 & 0.5 & 0.266 & 316 \\
\hline Cobalt & $* 24$ & $\bullet 0.29$ & 290 & 5.1 & 810 & $<4.4$ & - & 3.98 \\
\hline Copper & 18 & 12 & 3.873 & 0.23 & 5 & 0.205 & 0.26 & 8.6 \\
\hline Cyanide & 22 & 5.2 & 7.8 & - & 5.3 & - & 1.17 & 11 \\
\hline Fluorine & $* 3700$ & $* 190$ & $\bullet 8784$ & 4400 & $\bullet 5336$ & 3706 & - & 1080 \\
\hline Iron & - & 1000 & - & 158 & - & 16 & - & - \\
\hline Lead & 82 & 3.2 & 18.88 & 12.26 & 22 & - & 0.35 & 71 \\
\hline Lithium & - & - & - & - & - & - & - & - \\
\hline Magnesium & $* 6.5$ & $* 0.16$ & - & - & - & - & - & - \\
\hline Manganese & $* 330$ & $* 11$ & 1770 & $<1100$ & 1270 & $<1100$ & - & 112 \\
\hline Mercury, inorganic & 2.4 & $1.3^{\mathrm{b}}$ & $<0.23$ & 0.96 & 0.87 & 0.87 & 0.18 & 0.32 \\
\hline Mercury, methyl & $* 0.016$ & 0.0003 & 0.52 & $<0.04$ & $<0.03$ & 0.87 & - & 0.28 \\
\hline Molybdenum & 2200 & $* 30$ & - & 880 & - & 360 & - & - \\
\hline Nickel & 1400 & 160 & $<35$ & $<5$ & 62 & 45 & $11^{\mathrm{a}}$ & 215 \\
\hline Niobium & - & - & - & - & - & - & - & - \\
\hline Potassium & *5.3 & ${ }^{*} 0.13$ & - & - & - & - & - & - \\
\hline Selenium & 260 & 35 & 88.32 & 91.65 & 40 & 25 & 2.60 & - \\
\hline Silver & 4.1 & $0.39 \mathrm{~g}$ & 0.12 & 2.6 & 0.20 & $<0.56$ & $0.14^{\mathrm{a}}$ & 0.32 \\
\hline Sodium & $* 19,000$ & $* 480$ & - & 885,049 & - & - & - & - \\
\hline Strontium & - & - & - & - & - & - & - & - \\
\hline Thallium & $* 28$ & 2.5 & 57 & 135 & 81 & 64 & - & 67 \\
\hline Thorium & - & - & - & - & - & - & - & - \\
\hline Tin & - & - & - & - & - & - & - & - \\
\hline Titanium & - & - & - & - & - & - & - & - \\
\hline
\end{tabular}


Table 1 (continuod)

\begin{tabular}{|c|c|c|c|c|c|c|c|c|}
\hline \multirow[t]{2}{*}{ Chemical } & \multicolumn{2}{|c|}{$\begin{array}{l}\text { NAWQ criteria } \\
\text { "advisory values }\end{array}$} & \multicolumn{2}{|c|}{$\begin{array}{l}\text { Lowest chronic } \\
\text { value for. }\end{array}$} & \multicolumn{2}{|c|}{$\begin{array}{c}\text { Lowest test } \mathrm{EC}_{20} \\
\text { value for. }\end{array}$} & \multirow{2}{*}{$\begin{array}{l}\text { SS test } \\
\mathrm{EC}_{20}\end{array}$} & \multirow{2}{*}{$\begin{array}{c}\text { Population } \\
\mathrm{EC}_{20}\end{array}$} \\
\hline & Acute & Chronic & Fish & Daphnids & Fish & Daphnids & & \\
\hline Uranium & 0.28 & 0.007 & 142 & - & 455 & - & - & 27 \\
\hline Vanadium & .44 & $* 3.0$ & 80 & $>940$ & 41 & 430 & - & 32 \\
\hline Zinc & 120 & 110 & 36.41 & 46.73 & 47 & - & 21 & 80 \\
\hline Zirconium & $\bullet 8.52$ & $\bullet 0.213$ & $• 548$ & - & 2396 & - & - & 251 \\
\hline \multicolumn{9}{|c|}{ Organics } \\
\hline Acenaphthene & $\cdot 29$ & $\cdot 2.2$ & 413 & .6646 & $<197$ & - & - & - \\
\hline Acetone & $\cdot 31,000$ & $* 770$ & $\cdot 507.640$ & $\cdot 3,114,182$ & $\bullet 161,867$ & - & - & 23714 \\
\hline Anthracene & $\cdot 0.00058$ & 0.00001 & $\bullet 0.09$ & $<2.1$ & $* 0.35$ & $>8.2$ & - & -- \\
\hline Benzene & $\bullet 130$ & $* 3.2$ & $\bullet 299$ & $>98,000$ & 21 & - & - & 229 \\
\hline Benzidene & $\bullet 6.0$ & $* 0.15$ & $\cdot 134$ & - & $* 158$ & - & - & 68 \\
\hline Benzo(a)anthracene & $\bullet 0.11$ & $\cdot 0.0027$ & - & $* 0.65$ & - & - & - & - \\
\hline Benzo(a)pyrene & $\bullet 0.053$ & $\bullet 0.0013$ & - & $\bullet 0.30$ & $>2.99$ & - & - & - \\
\hline Benzo(b)fluoranthene & - & - & - & - & - & - & - & - \\
\hline Benzo(g,h,i)perylene & - & - & - & - & - & - & - & - \\
\hline Benzo(k)fluoranthene & - & - & - & - & - & - & - & - \\
\hline Benzoic acid & $* 18$ & 0.45 & •12.976 & - & $\bullet 7409$ & - & - & 1259 \\
\hline Benzyl alcohol & $\bullet 6.0$ & 0.147 & .589 & - & $* 550$ & - & - & 375 \\
\hline BHC (lindane) & 2.0 & 0.08 & 14.6 & 14.5 & $<1.1$ & 11 & 0.11 & - \\
\hline BHC (other) & "9.5 & $* 0.24$ & - & .95 & - & - & - & - \\
\hline \multicolumn{9}{|l|}{$\begin{array}{l}\text { BHC (other) } \\
\text { Bis(2-ethylhexyl) }\end{array}$} \\
\hline phthalate & $\cdot 3.2$ & ${ }^{*} 0.01$ & 8.4 & $<3$ & $>54$ & $<3$ & - & 50 \\
\hline \multicolumn{9}{|l|}{$\begin{array}{l}\text { phthalate } \\
\text { Butane, }\end{array}$} \\
\hline 1,1 -oxybis & - & - & - & - & - & - & - & - \\
\hline (2,1-ethanediyloxy)bis & - & - & - & - & - & - & - & - \\
\hline \multirow[t]{2}{*}{ 2-Butanone } & $* 63,000$ & $* 1600$ & $\cdot 282,170$ & $\bullet 1,394,927$ & $* 98,772$ & - & - & 17783 \\
\hline & - & - & - & - & - & - & - & - \\
\hline \multicolumn{8}{|l|}{ 4-chloro-4,4-difluoro- } & 1000 \\
\hline Carbon disulfide & 36 & 0.91 & $\bullet 9538$ & $\bullet 244$ & •5719 & - & - & $\begin{array}{r}1000 \\
224\end{array}$ \\
\hline Carbon tetrachloride & 690 & *63 & $>3400$ & $\bullet 5580$ & 65 & - & - & 224 \\
\hline Chlordane & 2.4 & $0.17^{b}$ & 1.6 & 16 & $<0.25$ & 12.1 & 0.50 & 0.71 \\
\hline Chlorobenzene & .460 & $\bullet 11.6$ & $\bullet 1203$ & $* 15,042$ & $* 1002$ & - & - & 165 \\
\hline Chloroethane & - & - & - & - & - & - & - & - \\
\hline Chloroform & $\bullet 570$ & $\bullet 14$ & *3578 & $\bullet 4483$ & 8400 & - & - & 562 \\
\hline
\end{tabular}


Table 1 (continued)

\begin{tabular}{|c|c|c|c|c|c|c|c|c|}
\hline \multirow[t]{2}{*}{ Chemical } & \multicolumn{2}{|c|}{$\begin{array}{l}\text { NAWQ criteria } \\
\text { advisory values }\end{array}$} & \multicolumn{2}{|c|}{$\begin{array}{l}\text { Lowest chronic } \\
\text { value for: }\end{array}$} & \multicolumn{2}{|c|}{$\begin{array}{l}\text { Lowest test } \mathrm{EC}_{20} \\
\text { value for. }\end{array}$} & \multirow{2}{*}{$\begin{array}{l}\text { SS test } \\
\mathrm{EC}_{20} \\
\end{array}$} & \multirow{2}{*}{$\begin{array}{c}\text { Population } \\
\mathrm{EC}_{20} \\
\end{array}$} \\
\hline & Acute & Chronic & Fish & Daphnids & Fish & Daphnids & & \\
\hline Chrysene & - & - & - & - & - & - & - & - \\
\hline \multicolumn{9}{|l|}{ DDD } \\
\hline $\mathrm{p}, \mathrm{p}^{\prime}$ & ${ }^{*} 0.017$ & ${ }^{*} 0.00042$ & $\bullet 1.69$ & - & *3.99 & - & - & 0.61 \\
\hline other & - & - & - & - & - & - & - & - \\
\hline DDT & 1.1 & ${ }^{*} 0.02^{\mathrm{c}}$ & $0.73^{d}$ & ${ }^{\star 0.016}$ & 0.35 & - & 0.008 & - \\
\hline Decane & $\bullet 190$ & $* 4.8$ & - & 7874 & - & - & - & - \\
\hline Di-n-butyl phthalate & $* 45$ & $* 4.4$ & $717^{e}$ & $717^{\mathrm{f}}$ & 270 & 500 & - & 251 \\
\hline Dibenzofuran & $* 80$ & 2.0 & - & $* 1003$ & - & - & - & - \\
\hline Dichlorodifluoromethane & - & - & - & - & - & - & - & - \\
\hline 1,1-Dichloroethane & $* 20$ & $* 0.51$ & $* 14,680$ & - & $\bullet 8219$ & - & - & 1585 \\
\hline 1,2-Dichloroethane & $\cdot 2300$ & $* 144$ & 41,364 & 15,200 & 29,000 & $<11,000$ & - & 1259 \\
\hline 1,1-Dichloroethene & .590 & $\bullet 15$ & $>2800$ & $* 4720$ & - & - & - & 447 \\
\hline 1,2-Dichloroethenes & $\bullet 14$ & $\bullet 0.34$ & *9538 & - & $* 5719$ & - & - & - \\
\hline \multicolumn{9}{|l|}{$\begin{array}{l}\text { 1,2-Dichloroethenes } \\
\text { 1,3-Dichloropropene }\end{array}$} \\
\hline trans- & - & - & - & - & - & - & - & - \\
\hline total & $\bullet 100$ & 2.6 & $* 345$ & 805 & $* 350$ & - & - & 40 \\
\hline Diethyll phthalate & $* 1100$ & $* 27$ & - & - & - & - & - & 1000 \\
\hline Di-n-octyl phthalate & - & - & 5700 & 570 & $<100$ & 310 & - & 1995 \\
\hline Dimethyl naphthalene & - & - & - & - & - & - & - & - \\
\hline $\begin{array}{l}\text { 4,6-Dinitro-ortho-cres } \\
\text { ol }\end{array}$ & - & - & - & - & - & - & - & - \\
\hline Ethenyl methyl benzene & - & - & - & - & - & - & - & - \\
\hline Ethyl benzene & ${ }^{*} 1100$ & *30 & $>440$ & $\bullet 12,922$ & - & - & - & 398 \\
\hline Ethyl methyl benzene & - & - & - & - & - & - & - & - \\
\hline Ethyll dimethyll benzene & - & - & - & - & - & - & - & - \\
\hline Fluoranthene & 69 & $* 1.7$ & - & $* 65,795$ & - & - & - & 32 \\
\hline Freon 113 & - & - & - & - & - & - & - & - \\
\hline Freon 123 & - & - & - & - & - & - & - & - \\
\hline Heptachlor & 0.52 & $\cdot 0.01^{\mathrm{c}}$ & 1.26 & $* 3.18$ & 0.86 & - & 0.004 & 0.1 \\
\hline Hexane & .82 & $\cdot 2.1$ & $\bullet 65,712$ & - & $\cdot 28,995$ & - & - & - \\
\hline 2,5-Hexanedione & - & - & - & - & - & - & - & - \\
\hline 2-Hexanone & 443 & $* 1.1$ & $* 32,783$ & - & $* 16,155$ & - & - & 1259 \\
\hline \multicolumn{9}{|l|}{$\begin{array}{l}\text { 2-Hexanone } \\
\text { 1-methylpentyl }\end{array}$} \\
\hline hydroperoxide & - & - & - & - & - & - & - & - \\
\hline
\end{tabular}


Table 1 (continuod)

\begin{tabular}{|c|c|c|c|c|c|c|c|c|}
\hline \multirow[t]{2}{*}{ Chemical } & \multicolumn{2}{|c|}{$\begin{array}{l}\text { N.AWQ criteria } \\
\text { "advisory values }\end{array}$} & \multicolumn{2}{|c|}{$\begin{array}{l}\text { Lowest chronic } \\
\text { value for. }\end{array}$} & \multicolumn{2}{|c|}{$\begin{array}{l}\text { Lowest test } \mathrm{EC}_{20} \\
\text { value for. }\end{array}$} & \multirow{2}{*}{$\begin{array}{l}\text { SS test } \\
\mathrm{EC}_{20}\end{array}$} & \multirow{2}{*}{$\begin{array}{c}\text { Population } \\
\mathrm{EC}_{20} \\
\end{array}$} \\
\hline & Acute & Chronic & Fish & Daphnids & Fish & Daphnids & & \\
\hline Indeno(1,2,3-c,d)pyrene & - & - & - & - & - & - & - & - \\
\hline Methyl cyclopentane & - & - & - & - & - & - & - & - \\
\hline 1-Methyl naphthalene & ${ }^{*} 0.90$ & ${ }^{*} 0.023$ & $* 526$ & - & $\cdot 500$ & - & - & 31.62 \\
\hline 2-Methyl naphthalene & - & - & - & - & - & - & - & - \\
\hline 4-Methyl-2-pentanone & $* 51$ & 2.3 & 77400 & - & - & - & - & 1585 \\
\hline 2-Methyl phenol & $* 310$ & $* 7.7$ & $* 489$ & $\bullet 1316$ & $* 470$ & - & - & 74 \\
\hline Methyl propenyl benzene & - & - & - & - & - & - & - & - \\
\hline Methylpropyl benzene & - & - & - & - & - & - & - & - \\
\hline Methylene chloride & $* 4300$ & $* 220$ & 108,000 & $\bullet 42,667$ & 410 & - & - & 1259 \\
\hline Naphthalene & .55 & $* 2.1$ & 620 & 1163 & 450 & $>600$ & - & 1000 \\
\hline \multicolumn{9}{|l|}{$\begin{array}{l}\text { Naphthalene } \\
\text { Nitrophenols }\end{array}$} \\
\hline $4-$ & 260 & $* 15$ & $\bullet 481$ & 7100 & $* 464$ & 5000 & - & 60 \\
\hline total & $230^{h}$ & $150^{\mathrm{h}}$ & - & - & - & - & - & - \\
\hline $\begin{array}{l}\mathrm{N} \text {-Nitroso-di-n-propy } \\
\text { lamine }\end{array}$ & - & - & - & - & - & - & - & - \\
\hline $\mathrm{N}$-Nitrosodiphenylamine & $\cdot 100$ & 2.5 & $\bullet 332$ & $* 1042$ & $\cdot 39$ & - & - & 40 \\
\hline Nonane & - & - & - & - & - & - & - & - \\
\hline 3-Octanone & $\bullet 1400$ & $* 34$ & $\cdot 5449$ & $* 110,147$ & $* 3571$ & - & - & - \\
\hline 9-Octadecenamide, (z) & - & - & - & - & - & - & - & - \\
\hline \multicolumn{9}{|l|}{$\begin{array}{l}\text { 9-Octadecenamide, (z) } \\
\text { PCB's }\end{array}$} \\
\hline Aroclor 1221 & 0.12 & $* 0.0029$ & $* 60$ & - & $* 80$ & - & - & 10 \\
\hline Aroclor 1232 & $* 0.23$ & $\bullet 0.0058$ & $* 124$ & - & $* 148$ & - & - & 16 \\
\hline Aroclor 1242 & $* 0.21$ & $\bullet 0.01$ & 9.0 & - & $<2.9$ & - & - & 1.58 \\
\hline Aroclor 1248 & $* 0.004$ & $* 0.0002$ & 0.2 & 4.3 & 0.4 & 2.5 & - & 1.26 \\
\hline Aroclor 1254 & $* 0.37$ & $* 0.02$ & 1.0 & 2.1 & 0.52 & 1.2 & - & 0.63 \\
\hline Aroclor ${ }^{\circ} 1260$ & $* 4.5$ & $* 0.11$ & 2.3 & - & 2.1 & - & - & 316 \\
\hline 1-Pentano! & 240 & $* 5.9$ & $* 30,493$ & - & $* 15,200$ & - & - & 3548 \\
\hline Phenanthrene & $* 8.4$ & ${ }^{*} 0.43$ & - & 200 & - & 110 & - & - \\
\hline Phenol & $* 450$ & $* 21$ & 1369 & 2005 & $<230$ & - & - & 4467 \\
\hline 2-Propanol & $* 100$ & 2.6 & $\bullet 83,257$ & - & $* 35,381$ & - & - & 3162 \\
\hline Pyrene & - & - & - & - & - & - & - & - \\
\hline 1,1,2,2-Tetrachloroethane & $* 400$ & $* 31$ & 2400 & 9900 & 1400 & $<420$ & - & 1585 \\
\hline Tetrachloroethene & •170 & 8.8 & 840 & 750 & 500 & 510 & - & 50 \\
\hline Toluene & $* 420$ & $* 10.4$ & 1269 & $\cdot 25,229$ & $<26$ & - & - & 200 \\
\hline
\end{tabular}


Table 1 (continued)

\begin{tabular}{|c|c|c|c|c|c|c|c|c|}
\hline \multirow[t]{2}{*}{ Chemical } & \multicolumn{2}{|c|}{$\begin{array}{l}\text { NAWQ criteria } \\
\text { advisory values }\end{array}$} & \multicolumn{2}{|c|}{$\begin{array}{l}\text { Lowest chronic } \\
\text { value for. }\end{array}$} & \multicolumn{2}{|c|}{$\begin{array}{l}\text { Lowest test } \mathrm{EC}_{20} \\
\text { value for: }\end{array}$} & \multirow{2}{*}{$\begin{array}{l}\text { SS test } \\
\mathrm{EC}_{20}\end{array}$} & \multirow{2}{*}{$\begin{array}{l}\text { Population } \\
\mathrm{EC}_{20}\end{array}$} \\
\hline & Acute & Chronic & Fish & Daphnids & Fish & Daphnids & & \\
\hline 1,1,1-Trichloroethane & 24 & 1.4 & 3493 & 1770 & 2457 & 1300 & - & 251 \\
\hline 1,1,2-Trichloroethane & $* 1400$ & $* 92$ & 9400 & 18,400 & 14,800 & 13,000 & - & 15849 \\
\hline Trichloroethene & $\bullet 880$ & $* 55$ & 11,060 & $* 7257$ & 5758 & - & - & 232 \\
\hline Trichlorofluoromethane & - & - & - & - & - & - & - & - \\
\hline Trimethỵl benzene & - & - & - & - & - & - & - & - \\
\hline Vinyl acetate & $* 32$ & $\bullet 0.8$ & $* 810$ & - & $\cdot 718$ & - & - & 108 \\
\hline Vinyl chloride & $\cdot 38$ & $\bullet 0.95$ & *28,879 & - & $\bullet 14,520$ & - & - & - \\
\hline Xylene & 60 & $* 1.46$ & $* 62,308$ & - & 2680 & - & - & - \\
\hline
\end{tabular}

Xylene

a Study $\mathrm{LC}$, were used rather than species mean $\mathrm{LC}_{50} \mathrm{~s}$ so water hastess would correspond to $\mathrm{EC}_{20}$ values.

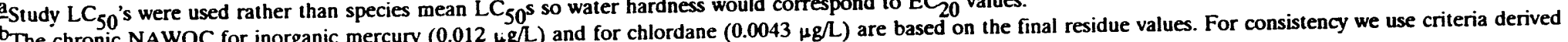
from the final chronic values.

CThe NAWQC for DDT $(0.001 \mu \mathrm{g} / \mathrm{L})$ and for Heptachlor $(0.0038 \mu \mathrm{g} / \mathrm{L})$ are based on the final residue values; for consistency we calculated approximated criteria for quatic life.

dThe MATC for DDT in Jarvinen et al. 1977 of $0.9 \mu \mathrm{g} / \mathrm{L}$ is the arithmetic mean of the NOEC and LOEC. We used the geometric mean which is 0.73 .

e fish di-n-butyl phthalate lowest chronic value, we used the geometric mean of the measured concentrations for the NOEC and LOEC rather than the nominal concentrations used by the authors. (McCarthy and Whitmore 1985)

fFor Daphnia magna di-n-butyl phthalate lowest chronic value, we used the mean of the geometric means of the NOEC and LOEC concentrations measured in fresh solutions and 1-d-old solutions. (McCarthy and Whitmore 1985)

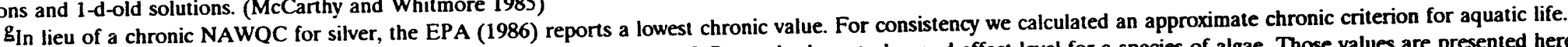

hin lieu of acute and chronic NAWOC, the EPA (1986) reports a lowest LC $_{50}$ and a lowest observed effect level for a species of algae. Those values are presented here because it was not judged to be appropriate to calculate estimated chronic values or advisory values to total nitrophenols. 


\subsection{ADVISORY VALUES}

NAWQC were not available for most chemicals. If NAWQC were not available for a chemical and could not be approximated, the method of Stephan and Erickson (n.d.) for calculating pesticide advisory values was applied. Advisory values are concentrations that would be expected to be higher than NAWQC in no more than $5 \%$ of cases. The advisory values are derived in a manner analogous to the criteria in that a number equivalent to the FAV, termed the "pesticide acute value" (PAV), is derived from acute toxicity data; it is multiplied by a number equivalent to the final acute-chronic ratio, termed the "pesticide acute-chronic ratio" (PACR), to obtain the chronic advisory values. PAVs are calculated by multiplying the lowest available $\mathrm{LC}_{50}$ by a factor derived from the distributions of lowest $\mathrm{LC}_{50}$ values from subsets of data to the PAVs derived from full data sets. The PACRs are calculated using all available and acceptable pairs of measured acute and chronic values, and factors oi 40 are used for missing chronic values (40 is a conservative default value supplied by Stephan and Erickson). The sources of data for the advisory values, the PAVs, and the PACRs are listed in Appendix A, and the factors used to calculate the PAVs are in Appendix B.

Knowledgeable readers may be aware of an EPA procedure for calculating advisory concentrations (OWRS \& ORD 1987). Advisory concentrations have the same purpose as the pesticide advisory values, but the statistical methods employed are not as defensible as those for the pesticide advisory values. The pesticide advisory values are not peculiarly applicable to pesticides, and in fact their statistical procedures were derived using all criteria chemicals. Therefore, in the remainder of this document they will be referred to simply as advisory values.

\subsection{LOWEST CHRONIC VALUES}

The lowest chronic values for lish and invertebrates reported in the literature are potential lower benchmarks. Chronic values are used to calculate the chronic NAWQC, but the lowest chronic value may be lower than the chronic NAWQC.

\subsection{ESTIMATED LOWEST CHRONIC VALUES}

Estimated lowest chronic values for lish and invertebrates are another set of potential lower benchmarks. Estimated chronic values were extrapolated from $96-\mathrm{h} \mathrm{LC}_{50}$ s using equations from Suter et al. (1987) and Suter (1992). The equations are as follows where $\mathrm{LC}_{50}$ equals the lowest species mean 96-h $\mathrm{LC}_{50}$ for fish and $48-\mathrm{h} \mathrm{EC}_{50}$ for daphnids and $\mathrm{CV}$ equals the estimated chronic value for that taxon. The $95 \%$ prediction interval at the mean is $\log \mathrm{CV} \pm$ the PI value (95\% prediction intervals contain $95 \%$ of observations versus $95 \%$ confidence intervals, which contain the mean with $95 \%$ confidence). 
Fish CV for a metallic contaminant:

$$
\begin{gathered}
\log C V=0.73 \log L_{C}-0.70 \\
P I=1.2
\end{gathered}
$$

Fish CV for a nonmetallic contaminant:

$$
\begin{gathered}
\log C V=1.07 \log L_{C} C_{50}-1.51 \\
P I=1.5
\end{gathered}
$$

Daphnid CV for a metallic contaminant:

$$
\begin{aligned}
& \log C V=0.96 \log \mathrm{LC}_{50}-1.08 \\
& \mathrm{PI}=1.56
\end{aligned}
$$

Daphnid CV for a nonmetallic contaminant:

$$
\begin{aligned}
\log C V= & 1.11 \log \mathrm{LC}_{50}-1.30 \\
& \mathrm{PI}=1.35
\end{aligned}
$$

\section{TEST $\mathrm{EC}_{20} \mathrm{~s}^{\mathrm{s}}$}

Another potential lower benchmark is the test $\mathrm{EC}_{20}$ for fish, which is defined as the highest tested concentration causing less than $20 \%$ reduction in (1) the weight of young fish per initial female fish in a life cycle or partial life cycle test or (2) the weight of young per egg in an early life-stage test. A similar potential lower benchmark is the test $\mathrm{EC}_{20}$ for daphnids, which is the highest tested concentration causing less than $20 \%$ reduction in the product of growth, fecundity, and survivorship in a chronic test with a daphnid species. (Daphnids include members of the genera Daphnia, Ceriodaphnia, and Simocephalus.) These benchmarks are intended to be indices of population production. They are equivalent to chronic values in that they are simply a summary of the results of chronic toxicity tests, and in most cases the same test supplied the lowest chronic value and the lowest test $\mathrm{EC}_{20}$. However, the test $\mathrm{EC}_{20} \mathrm{~s}$ are based on a level of biological effect rather than a level of statistical significance, and they integrate all of the stages of the toxicity test rather than treating each response independently. The $20 \%$ figure was chosen as approximately the mean level of effect observed at CVs and as a minimum detectable difference in population characteristics in the field (Suter et al. 1987, 1992).

\subsection{ESTIMATED TEST EC $_{20} \mathrm{~s}$}

The estimated test $\mathrm{EC}_{20}$ is another potential benchmark. The estimated values were extrapolated from $96-\mathrm{h} \mathrm{LC} \mathrm{L}_{50}$ values using equations from Suter (1992). The equation for the lowest fish test $\mathrm{EC}_{20}$ is as follows where $\mathrm{LC}_{50}$ equals the lowest species mean $96-\mathrm{h} \mathrm{LC}_{50}$ for fish and the $\mathrm{EC}_{25}$ for weight of juveniles per egg is used as an estimate of the test $\mathrm{EC}_{20}$ value. (The difference between $20 \%$ and $25 \%$ effect is trivial given the uncertainties in these 
estimates and the steepness of the concentration-response curves.) The log-scaled $95 \%$ prediction interval at the mean is $\log \mathrm{EC}_{25} \pm$ the PI value:

$$
\begin{aligned}
\log \mathrm{EC}_{25}= & 0.90 \log \mathrm{LC}_{50}-0.86 \\
& \mathrm{PI}=1.6
\end{aligned}
$$

\subsection{SENSITIVE SPECIES TEST EC $_{20}$ s}

The sixth potential benchmark is $\mathrm{EC}_{20}$ s, adjusted to approximate the fifth percentile of the species sensitivity distribution. It is calculated in the same way as the chronic NAWQC except that test $\mathrm{EC}_{20} \mathrm{~s}$ are used in place of CVs, and salt water species werc not included. The FAV for each of the criterion chemicals was divided by the geometric mean of ratios of $\mathrm{LC}_{50}$ s to $\mathrm{EC}_{20} \mathrm{~s}$. They are referred to as sensitive species (SS) test $\mathrm{EC}_{20} \mathrm{~s}$.

\subsection{POPULATION EC $20^{s}$}

The last potential benchmark is an estimate of the continuous concentration that would cause a $20 \%$ reduction in the recruit abundance of largemouth bass. The method used was described by Barnthouse et al. (1990) and is briefly summarized here. The recruit abundance estimates are generated by a matrix model of a reservoir largemouth bass population (Bartell 1990). The fecundity, hatching success, larval survival, and postlarval survival of the model population are each decremented by a value generated from statistical extrapolation models. For each life stage for which a concentration-response relationship could be calculated, that relationship was adjusted for the relative sensitivity of the test species and the bass. For those life stages with no concentration-response relationship, the relationship was estimated using life stage to life stage extrapolation models, and the taxonomic adjustment was made. However, if the authors of the study reported that life stage was unaffected, the decrement for that life stage was set to zero. If no chronic test data were available, extrapolations from $\mathrm{LC}_{50} \mathrm{~s}$ to chronic responses of each life stage were performed. Uncertainties in all of these extrapolations were propagated through the models to generate estimates of uncertainty. For each chemical, each available freshwater tish chronic test was used to parameterize a model run. If no chronic test data were available, each available freshwater fish $\mathrm{LC}_{50}$ was used to parameterize a model run. The results are presented in Table 2. The geometric mean of all population $\mathrm{EC}_{20}$ estimates for each chemical is reported in Table 1.

\subsection{BACKGROUND CONCENTRATIONS}

Background water concentrations should be used as a check for these benchmarks. That is, because some of these benchmarks are quite conservative and because the measured concentrations in ambient water may include forms that are not bioavailable, benchmark concentrations may be lower than background water concentrations. If the background concentrations are valid and represent an uncontaminated state, and if the background sites support normal biotic communities, then screening benchmarks lower than the background concentration should not be used. 
Table 2 Concentrations estimated to cause a $20 \%$ reduction in the recruit abundance of largemouth bass, with upper and lower $95 \%$ confidence bounds. All units are ug/L

\begin{tabular}{|c|c|c|c|c|c|c|}
\hline Chemical & Test species & $\begin{array}{l}\text { Test } \\
\text { type }\end{array}$ & $\begin{array}{l}\text { Lower } \\
95 \% \mathrm{CL}\end{array}$ & Median & $\begin{array}{l}\text { Upper } \\
95 \% \mathrm{CL}\end{array}$ & Source \\
\hline \multicolumn{7}{|c|}{ Inorganics } \\
\hline Ammonia & $\begin{array}{l}\text { Fathead minnow } \\
\text { Fathead minnow }\end{array}$ & $\begin{array}{l}\text { Chronic } \\
\text { Chronic }\end{array}$ & $\begin{array}{l}3.98 \\
3.98\end{array}$ & $\begin{array}{r}100 \\
32\end{array}$ & $\begin{array}{r}1585 \\
200\end{array}$ & $\begin{array}{l}\text { Mayes et al. } 1986 \\
\text { Thurston et al. } 1986\end{array}$ \\
\hline Antimony & Fathead minnow & Acute & 5.01 & 79 & 501 & EPA $1980 a$ \\
\hline Arsenic III & Fathead minnow & Chronic & 100 & 1995 & 31623 & Call et al. 1983 \\
\hline Arsenic V & $\begin{array}{l}\text { Fathead minnow } \\
\text { Rainbow trout } \\
\text { Mosquitofish }\end{array}$ & $\begin{array}{l}\text { Acute } \\
\text { Acute } \\
\text { Acute }\end{array}$ & $\begin{array}{l}20 \\
10 \\
32\end{array}$ & $\begin{array}{l}159 \\
100 \\
398\end{array}$ & $\begin{array}{r}1000 \\
501 \\
2512\end{array}$ & $\begin{array}{l}\text { EPA 1985a } \\
\text { EPA 1985a } \\
\text { EPA 1985a }\end{array}$ \\
\hline Beryllium & $\begin{array}{l}\text { Fathead minnow } \\
\text { Bluegill } \\
\text { Flagfish } \\
\text { Guppy }\end{array}$ & $\begin{array}{l}\text { Acute } \\
\text { Acute } \\
\text { Acute } \\
\text { Acute }\end{array}$ & $\begin{array}{l}0.35 \\
2.00 \\
2.00 \\
1.58\end{array}$ & $\begin{array}{l}7.08 \\
32 \\
32 \\
25\end{array}$ & $\begin{array}{r}40 \\
126 \\
126 \\
126\end{array}$ & $\begin{array}{l}\text { EPA } 1980 \mathrm{e} \\
\text { EPA 1980e } \\
\text { EPA } 1980 \mathrm{e} \\
\text { EPA } 1980 \mathrm{e}\end{array}$ \\
\hline Cadmium & $\begin{array}{l}\text { Fathead minnow } \\
\text { Bluegill } \\
\text { Brook trout } \\
\text { Brook trout } \\
\text { Flagfish } \\
\text { Flagfish }\end{array}$ & $\begin{array}{l}\text { Chronic } \\
\text { Chronic } \\
\text { Chronic } \\
\text { Chronic } \\
\text { Chronic } \\
\text { Chronic }\end{array}$ & $\begin{array}{l}1 \\
1.99 \\
0.13 \\
0.32 \\
0.63 \\
0.79\end{array}$ & $\begin{array}{l}10 \\
13 \\
1.26 \\
3.16 \\
3.98 \\
3.16\end{array}$ & $\begin{array}{l}79 \\
50 \\
6.31 \\
25 \\
50 \\
7.94\end{array}$ & $\begin{array}{l}\text { Pickering and Gast } 1972 \\
\text { Eaton } 1974 \\
\text { Benoit et al. } 1976 \\
\text { Sauter et al. } 1976 \\
\text { Carlson et al. } 1982 \\
\text { Spehai } 1976\end{array}$ \\
\hline Chromium III & Rainbow trout & Chronic & $\begin{array}{l}13 \\
0.79\end{array}$ & $\begin{array}{r}126 \\
3.16\end{array}$ & $\begin{array}{l}1000 \\
7.94\end{array}$ & Stevens and Chapman 1984 \\
\hline Chromium VI & $\begin{array}{l}\text { Fathead minnow } \\
\text { Rainbow trout }\end{array}$ & $\begin{array}{l}\text { Chronic } \\
\text { Chronic }\end{array}$ & $\begin{array}{r}25 \\
100\end{array}$ & $\begin{array}{l}158 \\
6.31\end{array}$ & $\begin{array}{l}1260 \\
5010\end{array}$ & $\begin{array}{l}\text { Pickering } 1980 \\
\text { Sauter et al. } 1976\end{array}$ \\
\hline Cobalt & Fathead minnow & Acute & 0.16 & 3.98 & 32 & Lind et al. 1978 \\
\hline
\end{tabular}


Table 2 (continued)

\begin{tabular}{|c|c|c|c|c|c|c|}
\hline Chemical & Test species & $\begin{array}{l}\text { Test } \\
\text { type }\end{array}$ & $\begin{array}{l}\text { Lower } \\
95 \% \mathrm{CL}\end{array}$ & Median & $\begin{array}{l}\text { Upper } \\
95 \% \mathrm{CL}\end{array}$ & Source \\
\hline Copper & $\begin{array}{l}\text { Fathead minnow } \\
\text { Fathead minnow } \\
\text { Bluegill } \\
\text { Brook trout } \\
\text { Brook trout } \\
\text { Coho salmon } \\
\text { Rainbow trout }\end{array}$ & $\begin{array}{l}\text { Chronic } \\
\text { Chronic } \\
\text { Chronic } \\
\text { Chronic } \\
\text { Chronic } \\
\text { Chronic } \\
\text { Chronic }\end{array}$ & $\begin{array}{l}0.40 \\
0.32 \\
1.995 \\
1 \\
1 \\
3.16 \\
3.98\end{array}$ & $\begin{array}{c}3.98 \\
3.16 \\
13 \\
3.98 \\
5.01 \\
32 \\
32\end{array}$ & $\begin{array}{r}40 \\
16 \\
50 \\
16 \\
40 \\
316 \\
200\end{array}$ & $\begin{array}{l}\text { Mount } 1968 \\
\text { Mount and Stephan } 1969 \\
\text { Benoit } 1975 \\
\text { McKim and Benoit } 1971 \\
\text { Sauter et al. 1976 } \\
\text { Hazel and Meith } 1970 \\
\text { Seim et al. } 1984\end{array}$ \\
\hline Cyanide & $\begin{array}{l}\text { Fathead minnow } \\
\text { Atlantic Salmon } \\
\text { Bluegill } \\
\text { Brook trout }\end{array}$ & $\begin{array}{l}\text { Chronic } \\
\text { Chronic } \\
\text { Chronic } \\
\text { Chronic }\end{array}$ & $\begin{array}{l}0.20 \\
1.99 \\
5.01 \\
1.58\end{array}$ & $\begin{array}{l}2.51 \\
16 \\
25 \\
13\end{array}$ & $\begin{array}{r}16 \\
200 \\
158 \\
79\end{array}$ & $\begin{array}{l}\text { Smith et al. } 1979 \\
\text { Leduc } 1978 \\
\text { Smith et al. } 1979 \\
\text { Smith et al. } 1979\end{array}$ \\
\hline Fluoride ion & $\begin{array}{l}\text { Fathead minnow } \\
\text { Rainbow trout } \\
\text { Brown trout }\end{array}$ & $\begin{array}{l}\text { Acute } \\
\text { Acute } \\
\text { Acute }\end{array}$ & $\begin{array}{r}56 \\
126 \\
79\end{array}$ & $\begin{array}{l}1000 \\
1259 \\
1000\end{array}$ & $\begin{array}{r}6310 \\
10000 \\
6310\end{array}$ & $\begin{array}{l}\text { Smith et al. } 1985 \\
\text { Smith et al. } 1985 \\
\text { AQUIRE* }\end{array}$ \\
\hline Lead & $\begin{array}{l}\text { Brook trout } \\
\text { Rainbow trout }\end{array}$ & $\begin{array}{l}\text { Chronic } \\
\text { Chronic }\end{array}$ & $\begin{array}{l}13 \\
13\end{array}$ & $\begin{array}{r}50 \\
100\end{array}$ & $\begin{array}{l}316 \\
631\end{array}$ & $\begin{array}{l}\text { Holcombe et al. } 1976 \\
\text { Sauter et al. } 1976\end{array}$ \\
\hline Manganese & Fathead minnow & Acute & 11 & 112 & 794 & Kimball n.d. \\
\hline Mercury, inorganic & $\begin{array}{l}\text { Fathead minnow } \\
\text { Fathead minnow }\end{array}$ & $\begin{array}{l}\text { Chronic } \\
\text { Chronic }\end{array}$ & $\begin{array}{l}0.04 \\
0.01\end{array}$ & $\begin{array}{l}0.79 \\
0.13\end{array}$ & $\begin{array}{l}13 \\
3.16\end{array}$ & $\begin{array}{l}\text { Call et al. } 1983 \\
\text { Snarski and Olson } 1982\end{array}$ \\
\hline Mercury, methyl & $\begin{array}{l}\text { Rainbow trout } \\
\text { Brook trout }\end{array}$ & $\begin{array}{l}\text { Acute } \\
\text { Acute }\end{array}$ & $\begin{array}{l}0.004 \\
0.02\end{array}$ & $\begin{array}{l}0.16 \\
0.50\end{array}$ & $\begin{array}{l}1.26 \\
3.98\end{array}$ & $\begin{array}{l}\text { EPA } 1985 c \\
\text { EPA } 1985 c\end{array}$ \\
\hline Nickel & $\begin{array}{l}\text { Fathead minnow } \\
\text { Rainbow trout } \\
\text { Rainbow trout }\end{array}$ & $\begin{array}{l}\text { Chronic } \\
\text { Chronic } \\
\text { Chronic }\end{array}$ & $\begin{array}{r}32 \\
13 \\
126\end{array}$ & $\begin{array}{r}126 \\
63 \\
1260\end{array}$ & $\begin{array}{r}501 \\
398 \\
15800\end{array}$ & $\begin{array}{l}\text { Pickering } 1974 \\
\text { Nebeker et al. } 1985 \\
\text { Nebeker et al. } 1985\end{array}$ \\
\hline
\end{tabular}


Table 2 (continued)

\begin{tabular}{|c|c|c|c|c|c|c|}
\hline Chemical & Test species & $\begin{array}{l}\text { Test } \\
\text { type }\end{array}$ & $\begin{array}{c}\text { Lower } \\
95 \% \mathrm{CL}\end{array}$ & Median & $\begin{array}{l}\text { Upper } \\
95 \% \mathrm{CL}\end{array}$ & Source \\
\hline Silver & $\begin{array}{l}\text { Fathead minnow } \\
\text { Rainbow trout }\end{array}$ & $\begin{array}{l}\text { Chronic } \\
\text { Chronic }\end{array}$ & $\begin{array}{l}0.01 \\
0.1\end{array}$ & $\begin{array}{l}0.1 \\
1\end{array}$ & $\begin{array}{l}1 \\
7.94\end{array}$ & $\begin{array}{l}\text { Holcombe et al. } 1983 \\
\text { Nebeker et al. } 1983\end{array}$ \\
\hline Thallium & $\begin{array}{l}\text { Fathead minnow } \\
\text { Bluegill }\end{array}$ & $\begin{array}{l}\text { Acute } \\
\text { Acute }\end{array}$ & $\begin{array}{l}0.25 \\
73\end{array}$ & $1000^{4.47}$ & $\begin{array}{r}40 \\
6310\end{array}$ & $\begin{array}{l}\text { EPA } 1980 x \\
\text { EPA } 1980 x\end{array}$ \\
\hline Uranium & $\begin{array}{l}\text { Fathead minnow } \\
\text { Fathead minnow } \\
\text { Fathead minnow } \\
\text { Fathead minnow }\end{array}$ & $\begin{array}{l}\text { Acute } \\
\text { Acute } \\
\text { Acute } \\
\text { Acute }\end{array}$ & $\begin{array}{l}0.398 \\
0.501 \\
0.631 \\
32\end{array}$ & $\begin{array}{r}10 \\
10 \\
13 \\
398\end{array}$ & $\begin{array}{r}50 \\
50 \\
79 \\
3980\end{array}$ & $\begin{array}{l}\text { Cushman et al. } 1977 \\
\text { Cushman et al. } 1977 \\
\text { Cushman et al. } 1977 \\
\text { Cushman et al. } 1977\end{array}$ \\
\hline Vanadium & $\begin{array}{l}\text { Fathead minnow } \\
\text { Brook trout } \\
\text { Flagfish }\end{array}$ & $\begin{array}{l}\text { Acute } \\
\text { Acute } \\
\text { Acute }\end{array}$ & $\begin{array}{l}0.28 \\
5.01 \\
10 \\
10\end{array}$ & $\begin{array}{l}5.01 \\
63 \\
100 \\
100\end{array}$ & $\begin{array}{r}40 \\
398 \\
501 \\
501\end{array}$ & $\begin{array}{l}\text { Kimball n.d. } \\
\text { Ernst and Garside } 1987 \\
\text { Holdway and Sprague } \\
1979\end{array}$ \\
\hline Zinc & $\begin{array}{l}\text { Fathead minnow } \\
\text { Fathead minnow } \\
\text { Coho salmon } \\
\text { Flagfish } \\
\text { Rainbow trout }\end{array}$ & $\begin{array}{l}\text { Chronic } \\
\text { Chronic } \\
\text { Chronic } \\
\text { Chronic } \\
\text { Chronic }\end{array}$ & $\begin{array}{l}3.98 \\
10 \\
3.16 \\
16 \\
16 \\
6.31 \\
40\end{array}$ & $\begin{array}{r}40 \\
100 \\
40 \\
126 \\
126 \\
32 \\
501\end{array}$ & $\begin{array}{r}316 \\
501 \\
316 \\
794 \\
794 \\
126 \\
5010\end{array}$ & $\begin{array}{l}\text { Benoit and Holcombe } \\
1978 \\
\text { Brungs } 1969 \\
\text { Finlayson and Verrue } \\
1980 \\
\text { Spehar } 1976 \\
\text { Sinley et al. } 1974\end{array}$ \\
\hline Zirconium & $\begin{array}{l}\text { Fathead minnow } \\
\text { Fathead minnow } \\
\text { Fathead minnow } \\
\text { Fathead minnow } \\
\text { Bluegill } \\
\text { Bluegill }\end{array}$ & $\begin{array}{l}\text { Acute } \\
\text { Acute } \\
\text { Acute } \\
\text { Acute } \\
\text { Acute } \\
\text { Acute }\end{array}$ & $\begin{array}{c}3.16 \\
3.16 \\
32 \\
63 \\
10 \\
200\end{array}$ & $\begin{array}{r}50 \\
50 \\
398 \\
794 \\
126 \\
2510\end{array}$ & $\begin{array}{r}316 \\
398 \\
3160 \\
6310 \\
501 \\
15800\end{array}$ & $\begin{array}{l}\text { Cushman et al. } 1977 \\
\text { Cushman et al. } 1977 \\
\text { Cushman et al. } 1977 \\
\text { Cushman et al. } 1977 \\
\text { Cushman et al. } 1977 \\
\text { Cushman et al. } 1977\end{array}$ \\
\hline
\end{tabular}


Table 2 (continued)

\begin{tabular}{|c|c|c|c|c|c|c|}
\hline Chemical & Test species & $\begin{array}{l}\text { Test } \\
\text { type }\end{array}$ & $\begin{array}{l}\text { Lower } \\
95 \% \mathrm{CL}\end{array}$ & Median & $\begin{array}{l}\text { Upper } \\
95 \% \mathrm{CL}\end{array}$ & Source \\
\hline \multicolumn{7}{|c|}{$\underline{\text { Organics }}$} \\
\hline AC 222,705 & Fathead minnow & Chronic & 0.0006 & 0.01 & 0.063 & Spehar et al. 1983 \\
\hline Acetone & $\begin{array}{l}\text { Fathead minnow } \\
\text { Bluegill } \\
\text { Rainbow trout } \\
\text { Mosquitofish }\end{array}$ & $\begin{array}{l}\text { Acute } \\
\text { Acute } \\
\text { Acute } \\
\text { Acute }\end{array}$ & $\begin{array}{r}1780 \\
3160 \\
1260 \\
631\end{array}$ & $\begin{array}{c}19900 \\
50100 \\
31600 \\
10000\end{array}$ & $\begin{array}{r}25100 \\
501000 \\
316000 \\
100000\end{array}$ & $\begin{array}{l}\text { AQUIRE* } \\
\text { AQUIRE* } \\
\text { AQUIRE* } \\
\text { AQUIRE* }\end{array}$ \\
\hline AG thiosulfate complex & Fathead minnow & Chronic & 1000 & 10000 & 79400 & LeBlanc et al. 1984 \\
\hline Aldicarb & Fathead minnow & Chronic & $\begin{array}{l}3.16 \\
631\end{array}$ & $\begin{array}{r}50 \\
10000\end{array}$ & $\begin{array}{r}631 \\
100000\end{array}$ & $\begin{array}{l}\text { Pickering and Giliam } \\
1982\end{array}$ \\
\hline Atrazine & Brook trout & Chronic & 100 & 1000 & 12600 & Macek et al. 1976b \\
\hline Benzene & $\begin{array}{l}\text { Fathead minnow } \\
\text { Bluegill } \\
\text { Rainbow trout } \\
\text { Mosquitofish } \\
\text { Guppy }\end{array}$ & $\begin{array}{l}\text { Acute } \\
\text { Acute } \\
\text { Acute } \\
\text { Acute } \\
\text { Acute }\end{array}$ & $\begin{array}{c}10 \\
13 \\
3.16 \\
200 \\
32\end{array}$ & $\begin{array}{r}100 \\
158 \\
40 \\
3160 \\
316\end{array}$ & $\begin{array}{r}794 \\
1000 \\
316 \\
25100 \\
1580\end{array}$ & $\begin{array}{l}\text { EPA } 1980 c \\
\text { EPA } 1980 c \\
\text { EPA 1980c } \\
\text { EPA } 1980 c \\
\text { EPA } 1980 c\end{array}$ \\
\hline Benzidene & $\begin{array}{l}\text { Rainbow trout } \\
\text { Lake trout } \\
\text { Flagfish }\end{array}$ & $\begin{array}{l}\text { Acute } \\
\text { Acute } \\
\text { Acute }\end{array}$ & $\begin{array}{l}5.01 \\
3.16 \\
10\end{array}$ & $\begin{array}{r}63 \\
40 \\
126\end{array}$ & $\begin{array}{l}398 \\
251 \\
794\end{array}$ & $\begin{array}{l}\text { EPA 1980b } \\
\text { EPA 1980b } \\
\text { EPA 1980b }\end{array}$ \\
\hline Benzoic acid & Mosquitofish & Acute & 126 & 1260 & 10000 & AQUIRE* \\
\hline Benzyl alcohol & $\begin{array}{l}\text { Fathead minnow } \\
\text { Bluegill }\end{array}$ & $\begin{array}{l}\text { Acute } \\
\text { Acute }\end{array}$ & $\begin{array}{l}16 \\
6.31\end{array}$ & $\begin{array}{r}1780 \\
79\end{array}$ & $\begin{array}{r}12600 \\
398\end{array}$ & $\begin{array}{l}\text { AQUIRE* } \\
\text { AQUIRE* }\end{array}$ \\
\hline
\end{tabular}


Table 2 (continued)

\begin{tabular}{|c|c|c|c|c|c|c|}
\hline Chemical & Test species & $\begin{array}{l}\text { Test } \\
\text { type }\end{array}$ & $\begin{array}{c}\text { Lower } \\
\text { 95\% CL } \\
\end{array}$ & Median & $\begin{array}{c}\text { Upper } \\
95 \% \mathrm{CL} \\
\end{array}$ & Source \\
\hline Bis(2-ethylhexyl) phthalate & $\begin{array}{l}\text { Rainbow trout } \\
\text { Largemouth bass } \\
\text { Channel catfish }\end{array}$ & $\begin{array}{l}\text { Acute } \\
\text { Acute } \\
\text { Acute }\end{array}$ & $\begin{array}{l}100 \\
25 \\
0.006\end{array}$ & $\begin{array}{c}1000 \\
316 \\
0.40\end{array}$ & $\begin{array}{l}7940 \\
1580 \\
6.31\end{array}$ & $\begin{array}{l}\text { AQUIRE* } \\
\text { AQUIRE* } \\
\text { AQUIRE* }\end{array}$ \\
\hline 2-Butanone & $\begin{array}{l}\text { Fathead minnow } \\
\text { Mosquitofish }\end{array}$ & $\begin{array}{l}\text { Acute } \\
\text { Acute }\end{array}$ & $\begin{array}{r}501 \\
1990\end{array}$ & $\begin{array}{l}10000 \\
31600\end{array}$ & $\begin{array}{l}100000 \\
398000\end{array}$ & $\begin{array}{l}\text { AQUIRE* } \\
\text { AQUIRE* }\end{array}$ \\
\hline Captan & Fathead minnow & Chronic & 0.32 & 3.16 & 20 & Hermanutz et al. 1973 \\
\hline Carbaryl & Fathead minnow & Chronic & 3.16 & 32 & 1000 & Carlson 1971 \\
\hline Carbon disulfide & Mosquitofish & Acute & 100 & 1000 & 7940 & AQUIRE* \\
\hline Carbon tetrachloride & $\begin{array}{l}\text { Fathead minnow } \\
\text { Bluegill }\end{array}$ & $\begin{array}{l}\text { Acute } \\
\text { Acute }\end{array}$ & $\begin{array}{l}11 \\
40\end{array}$ & $\begin{array}{l}126 \\
398\end{array}$ & $\begin{array}{l}1260 \\
3160\end{array}$ & $\begin{array}{l}\text { EPA } 1980 f \\
\text { EPA } 1980 f\end{array}$ \\
\hline Chloramine & Fathead minnow & Chronic & 0.32 & 3.16 & 13 & Arthur and Eaton 1971 \\
\hline Chlordane & $\begin{array}{l}\text { Bluegill } \\
\text { Brook trout }\end{array}$ & $\begin{array}{l}\text { Chronic } \\
\text { Chronic }\end{array}$ & $\begin{array}{l}0.13 \\
0.20\end{array}$ & $\begin{array}{l}0.40 \\
1.26\end{array}$ & $\begin{array}{l}2.51 \\
7.94\end{array}$ & $\begin{array}{l}\text { Cardwell et al. } 1977 \\
\text { Cardwell et al. } 1977\end{array}$ \\
\hline Chlorobenzene & $\begin{array}{l}\text { Fathead minnow } \\
\text { Bluegill } \\
\text { Guppy }\end{array}$ & $\begin{array}{l}\text { Acute } \\
\text { Acute } \\
\text { Acute }\end{array}$ & $\begin{array}{l}11 \\
10 \\
32\end{array}$ & $\begin{array}{l}112 \\
126 \\
316\end{array}$ & $\begin{array}{r}794 \\
1000 \\
2510\end{array}$ & $\begin{array}{l}\text { EPA } 1980 \mathrm{~g} \\
\text { EPA } 1980 \mathrm{~g} \\
\text { EPA } 1980 \mathrm{~g}\end{array}$ \\
\hline Chloroform & $\begin{array}{l}\text { Bluegill } \\
\text { Rainbow trout }\end{array}$ & $\begin{array}{l}\text { Acute } \\
\text { Acute }\end{array}$ & $\begin{array}{l}79 \\
40\end{array}$ & $\begin{array}{l}794 \\
398\end{array}$ & $\begin{array}{l}5010 \\
3160\end{array}$ & $\begin{array}{l}\text { EPA } 1980 \mathrm{i} \\
\text { EPA } 1980 \mathrm{i}\end{array}$ \\
\hline$p, p ' D D D$ & $\begin{array}{l}\text { Fathead minnow } \\
\text { Bluegill } \\
\text { Rainbow trout } \\
\text { Largemouth bass } \\
\text { Channel catfish }\end{array}$ & $\begin{array}{l}\text { Acute } \\
\text { Acute } \\
\text { Acute } \\
\text { Acute } \\
\text { Acute }\end{array}$ & $\begin{array}{l}0.79 \\
0.002 \\
0.02 \\
0.003 \\
0.025\end{array}$ & $\begin{array}{l}13 \\
0.1 \\
0.40 \\
0.13 \\
1.26\end{array}$ & $\begin{array}{c}100 \\
1 \\
3.16 \\
1 \\
16\end{array}$ & $\begin{array}{l}\text { AQUIRE* } \\
\text { AQUIRE* } \\
\text { AQUIRE* } \\
\text { AQUIRE* } \\
\text { AQUIRE* }\end{array}$ \\
\hline
\end{tabular}


Table 2 (continued)

\begin{tabular}{|c|c|c|c|c|c|c|}
\hline Chemical & Test species & $\begin{array}{l}\text { Test } \\
\text { type }\end{array}$ & $\begin{array}{l}\text { Lower } \\
95 \% \mathrm{CL}\end{array}$ & Median & $\begin{array}{l}\text { Upper } \\
95 \% \mathrm{CL}\end{array}$ & Source \\
\hline 2,4-D Butoxyethanol ester & Coho salmon & Chronic & $\begin{array}{l}10 \\
0.025\end{array}$ & $\begin{array}{r}100 \\
50\end{array}$ & $\begin{array}{r}1260 \\
16\end{array}$ & $\begin{array}{l}\text { Finlayson and Verrue } \\
1985\end{array}$ \\
\hline Diazinon & $\begin{array}{l}\text { Fathead minnow } \\
\text { Flagfish }\end{array}$ & $\begin{array}{l}\text { Chronic } \\
\text { Chronic }\end{array}$ & $\begin{array}{l}0.32 \\
0.025 \\
10 \\
10\end{array}$ & $\begin{array}{c}3.16 \\
3980 \\
50 \\
50\end{array}$ & $\begin{array}{r}25 \\
16 \\
316 \\
316\end{array}$ & $\begin{array}{l}\text { Allison and Hermanutz } \\
1977 \\
\text { Allison and Hermanutz } \\
1977\end{array}$ \\
\hline Di-n-butyl phthalate & Fathead minnow & Chronic & $\begin{array}{l}13 \\
10\end{array}$ & $\begin{array}{r}251 \\
3980\end{array}$ & $\begin{array}{r}3980 \\
316\end{array}$ & $\begin{array}{l}\text { McCarthy and Whitmore } \\
1985\end{array}$ \\
\hline 2,6-Dichlorobenzamide & Rainbow trout & Chronic & 1260 & 12600 & 50100 & Van Leeuwen and Maas 1985 \\
\hline 1,3-Dichlorobenzene & Fathead minnow & Chronic & 316 & 1585 & 15849 & Ahmed et al. 1984 \\
\hline 1,4-Dichlorobenzene & Fathead minnow & Chronic & 32 & 398 & 6310 & Ahmed et al. 1984 \\
\hline 1,1-Dichloroethane & Guppy & Acute & 158 & 1580 & 12600 & AQUIRE* \\
\hline 1,2-Dichloroethane & $\begin{array}{l}\text { Fathead minnow } \\
\text { Bluegill }\end{array}$ & $\begin{array}{l}\text { Acute } \\
\text { Acute }\end{array}$ & $\begin{array}{r}40 \\
316\end{array}$ & $\begin{array}{r}398 \\
3980\end{array}$ & $\begin{array}{r}3160 \\
31600\end{array}$ & $\begin{array}{l}\text { EPA } 1980 \mathrm{~h} \\
\text { EPA } 1980 \mathrm{~h}\end{array}$ \\
\hline 1,1-Dichloroethene & $\begin{array}{l}\text { Fathead minnow } \\
\text { Bluegill }\end{array}$ & $\begin{array}{l}\text { Acute } \\
\text { Acute }\end{array}$ & $\begin{array}{l}40 \\
50\end{array}$ & $\begin{array}{l}398 \\
501\end{array}$ & $\begin{array}{l}3160 \\
3980\end{array}$ & $\begin{array}{l}\text { EPA 1980k } \\
\text { EPA 1980k }\end{array}$ \\
\hline 2,4-Dichlorophenol & Fathead minnow & Chronic & 32 & 200 & 1580 & Holcombe et al. 1982 \\
\hline 1,2-Dichloropropane & Fathead minnow & Chronic & 398 & 3980 & 39800 & Benoit et al. 1982 \\
\hline 1,3-Dichloropropane & Fathead minnow & Chronic & 501 & 5010 & 50100 & Benoit et al. 1982 \\
\hline 1,3-Dichloropropene & Bluegill & Acute & 3.16 & 40 & 251 & EPA 19801 \\
\hline Diethyl phthalate & Bluegill & Acute & 79 & 1000 & 6310 & AQUIRE* \\
\hline 2,4-Dimethylphenol & Fathead minnow & Chronic & 100 & 1260 & 19900 & Holcombe et al. 1982 \\
\hline
\end{tabular}


Table 2 (continued)

\begin{tabular}{|c|c|c|c|c|c|c|}
\hline Chemical & Test species & $\begin{array}{l}\text { Test } \\
\text { type }\end{array}$ & $\begin{array}{c}\text { Lower } \\
95 \% \mathrm{CL} \\
\end{array}$ & Median & $\begin{array}{c}\text { Upper } \\
95 \% \mathrm{CL}\end{array}$ & Source \\
\hline Dimethyl phthalate & Rainbow trout & Chronic & 0.004 & 0.04 & 0.40 & Ward and Boeri 1991b \\
\hline 1,3-Dinitrobenzene & $\begin{array}{l}\text { Rainbow trout } \\
\text { Rainbow trout }\end{array}$ & $\begin{array}{l}\text { Chronic } \\
\text { Chronic }\end{array}$ & $\begin{array}{r}100 \\
63\end{array}$ & $\begin{array}{r}1000 \\
794\end{array}$ & $\begin{array}{l}7940 \\
6310\end{array}$ & $\begin{array}{l}\text { Van Der Schalie } 1983 \\
\text { Van Der Schalie } 1983\end{array}$ \\
\hline Dinoseb & Fathead minnow & Chronic & 0.13 & 3.16 & 40 & Call et al. 1983 \\
\hline Di-n-octyl phthalate & Fathead minnow & Chronic & $\begin{array}{r}200 \\
63\end{array}$ & $\begin{array}{r}1990 \\
794\end{array}$ & $\begin{array}{r}39800 \\
316\end{array}$ & $\begin{array}{l}\text { McCarthy and Whitmore } \\
1985\end{array}$ \\
\hline Diuron & Fathead minnow & Chronic & 1 & 16 & 158 & Call et al. 1983 \\
\hline Dursban & Fathead minnow & Chronic & 0.002 & 0.032 & 0.20 & Jarvinen et al. 1983 \\
\hline DNBP & Rainbow trout & Chronic & 0.00004 & 0.0004 & 0.005 & Ward and Boeri 1991a \\
\hline Endrin & Fathead minnow & Chronic & 0.005 & 0.13 & 1.58 & Carlson et al. 1982 \\
\hline Enolsufan & Fathead minnow & Chronic & 0.002 & 0.016 & 0.13 & Macek et al. 1976b \\
\hline Ethyl benzene & $\begin{array}{l}\text { Fathead minnow } \\
\text { Bluegill } \\
\text { Guppy }\end{array}$ & $\begin{array}{l}\text { Acute } \\
\text { Acute } \\
\text { Acute }\end{array}$ & $\begin{array}{l}10 \\
50 \\
79\end{array}$ & $\begin{array}{l}158 \\
501 \\
794\end{array}$ & $\begin{array}{l}1000 \\
3980 \\
5010\end{array}$ & $\begin{array}{l}\text { EPA } 1980 \mathrm{~m} \\
\text { EPA } 1980 \mathrm{~m} \\
\text { EPA } 1980 \mathrm{~m}\end{array}$ \\
\hline Fenitrothion & Fathead minnow & Chronic & 0.1 & 126 & 1990 & Kleiner et al. 1984 \\
\hline Fluoranthene & Bluegill & Acute & 2.00 & 32 & 126 & EPA 1980n \\
\hline Fluridone & Fathead minnow & Chronic & 32 & 398 & 10000 & Hamelink et al. 1986 \\
\hline Fonofos & Fathead minnow & Chronic & $\begin{array}{l}3.16 \\
79\end{array}$ & $\begin{array}{l}20 \\
79\end{array}$ & $\begin{array}{r}158 \\
6310\end{array}$ & $\begin{array}{l}\text { Pickering and Giliam } \\
1982\end{array}$ \\
\hline Guthion & Fathead minnow & Chronic & 0.013 & 0.13 & 3.98 & Adelman et al. 1976 \\
\hline Heptachlor & Fathead minnow & Chronic & 0.01 & 0.1 & 0.63 & Macek et al. 1976b \\
\hline
\end{tabular}


Table 2 (continued)

\begin{tabular}{|c|c|c|c|c|c|c|}
\hline Chemical & Test species & $\begin{array}{l}\text { Test } \\
\text { type }\end{array}$ & $\begin{array}{l}\text { Lower } \\
95 \% \mathrm{CL}\end{array}$ & Median & $\begin{array}{c}\text { Upper } \\
95 \% \mathrm{CL}\end{array}$ & Source \\
\hline Hexachlorobutadiene & Fathead minnow & Chronic & 0.32 & 3.98 & 63 & Benoit et al. 1982 \\
\hline Hexachlorocyclohexane(lindane) & Bluegill & Chronic & 0.16 & 1 & 6.31 & Macek et al. 1976a \\
\hline Hexachloroethane & Fathead minnow & Chronic & 3.98 & 100 & 1580 & Ahmed et al. 1984 \\
\hline 2-Hexanone & Fathead minnow & Acute & 100 & 1260 & 12600 & AQUIRE* \\
\hline Kelthane & Fathead minnow & Chronic & 0.32 & 7.94 & 100 & Spehar et al. 1982 \\
\hline LAS 11.2 & Fathead misnow & Chronic & 126 & 1580 & 25100 & Holman et al. 1980 \\
\hline LAS 11.7 & Fathead minnow & Chronic & 13 & 200 & 2510 & Holman et al. 1980 \\
\hline LAS Mixture & Fathead minnow & Chronic & $\begin{array}{l}20 \\
79\end{array}$ & $\begin{array}{r}316 \\
79\end{array}$ & $\begin{array}{l}1580 \\
6310\end{array}$ & $\begin{array}{l}\text { Pickering and Thatcher } \\
1970\end{array}$ \\
\hline Malathion & Flagfish & Chronic & 3.162 & 20 & 126 & Hermanutz 1978 \\
\hline 1-Methylnaphthalene & Fathead minnow & Acute & 1.78 & 31.62 & 200 & AQUIRE* \\
\hline 4-Methyl-2-pentanone & Fathead minnow & Acute & 141 & 1580 & 15800 & AQUIRE* \\
\hline 2-Methylphenol & $\begin{array}{l}\text { Fathead minnow } \\
\text { Bluegill } \\
\text { Rainbow trout }\end{array}$ & $\begin{array}{l}\text { Acute } \\
\text { Acute } \\
\text { Acute }\end{array}$ & $\begin{array}{c}3.98 \\
13 \\
6.31\end{array}$ & $\begin{array}{r}40 \\
126 \\
79\end{array}$ & $\begin{array}{r}398 \\
1000 \\
398\end{array}$ & $\begin{array}{l}\text { AQUIRE* } \\
\text { AQUIRE* } \\
\text { AQUIRE* }\end{array}$ \\
\hline Methylene chloride & $\begin{array}{l}\text { Fathead minnow } \\
\text { Bluegill }\end{array}$ & $\begin{array}{l}\text { Acute } \\
\text { Acute }\end{array}$ & $\begin{array}{l}63.10 \\
158\end{array}$ & $\begin{array}{l}1000 \\
1580\end{array}$ & $\begin{array}{l}10000 \\
12600\end{array}$ & $\begin{array}{l}\text { AQUIRE* } \\
\text { AQUIRE* }\end{array}$ \\
\hline Naphthalene & Fathead minnow & Chronic & 73 & 1000 & 12600 & Degraeve et al. 1982 \\
\hline 4-Nitrophenol & $\begin{array}{l}\text { Fathead minnow } \\
\text { Bluegiil } \\
\text { Rainbow trout } \\
\text { Channel catfish }\end{array}$ & $\begin{array}{l}\text { Acute } \\
\text { Acute } \\
\text { Acute } \\
\text { Acute }\end{array}$ & $\begin{array}{l}13 \\
3.98 \\
6.31 \\
0.79\end{array}$ & $\begin{array}{r}159 \\
50 \\
79 \\
20\end{array}$ & $\begin{array}{r}1260 \\
316 \\
398 \\
200\end{array}$ & $\begin{array}{l}\text { AQUIRE* } \\
\text { AQUIRE* } \\
\text { AQUIRE* } \\
\text { AQUIRE* }\end{array}$ \\
\hline
\end{tabular}


Table 2 (continued)

\begin{tabular}{lllcrrr}
\multicolumn{1}{c}{ Chemical } & \multicolumn{1}{c}{ Test species } & $\begin{array}{c}\text { Test } \\
\text { type }\end{array}$ & $\begin{array}{c}\text { Lower } \\
95 \% \text { CL }\end{array}$ & Median & $\begin{array}{c}\text { Upper } \\
95 \% \text { CL }\end{array}$ & Source \\
\hline N-nitrosodiphenylamine & Bluegill & Acute & 3.16 & 40 & 251 & AQUIRE* $^{*}$ \\
PCB's: Aroclor 1221 & Cutthroat trout & Acute & 0.63 & 10 & 50 & AQUIRE* \\
PCB's: Aroclor 1232 & Cutthroat trout & Acute & 1.26 & 16 & 126 & AQUIRE* \\
PCB's: Aroclor 1242 & Fathead minnow & Chronic & 0.13 & 1.58 & 40 & Nebeker et al. 1974 \\
PCB's: Aroclor 1248 & Flagfish & Chronic & 0.13 & 1.26 & 7.94 & Nebeker et al. 1974 \\
PCB's: Aroclor 1254 & Fathead minnow & Chronic & 0.1 & 0.63 & 7.94 & Nebeker et al. 1974 \\
PCB's: Aroclor 1260 & Cutthroat trout & Acute & 32 & 316 & 2510 & AQUIRE* \\
Pentachloroethane & Fathead minnow & Chronic & 100 & 1260 & 19900 & Ahmed et al. 1984 \\
Pentachlorophenol & Fathead minnow & Chronic & 1.58 & 32 & 501 & Holcombe et al. 1982 \\
& Fathead minnow & Chronic & 0.32 & 1.99 & 20 & Spehar et al. 1985 \\
& Fathead minnow & Chronic & 0.63 & 6.31 & 63 & Spehar et al. 1985 \\
& Fathead minnow & Chronic & 1.26 & 13 & 126 & Spehar et al. 1985 \\
& Fathead minnow & Chronic & 1.99 & 16 & 158 & Spehar et al. 1985 \\
& Rainbow trout & Chronic & 1.58 & 10 & 50 & Dominguez and Chapman \\
& & & 1.58 & 10 & 50 & 1984 \\
1-Pentanol & Bluegill & Acute & 398 & 5010 & 39800 & AQUIRE* \\
& Rainbow trout & Acute & 200 & 2510 & 19900 & AQUIRE*
\end{tabular}


Table 2 (continued)

\begin{tabular}{|c|c|c|c|c|c|c|}
\hline Chemical & Test species & $\begin{array}{l}\text { Test } \\
\text { type }\end{array}$ & $\begin{array}{l}\text { Lower } \\
95 \% \mathrm{CL}\end{array}$ & Median & $\begin{array}{c}\text { Upper } \\
95 \% \mathrm{CL}\end{array}$ & Source \\
\hline Phenol & $\begin{array}{l}\text { Fathead minnow } \\
\text { Rainbow trout }\end{array}$ & $\begin{array}{l}\text { Chronic } \\
\text { Chronic }\end{array}$ & $\begin{array}{r}1000 \\
126\end{array}$ & $\begin{array}{r}12600 \\
1580\end{array}$ & $\begin{array}{r}199000 \\
15800\end{array}$ & $\begin{array}{l}\text { Degraeve ei al. } 1980 \\
\text { Degraeve et al. } 1980\end{array}$ \\
\hline Propanil & Fathead minnow & Chronic & 0.01 & 0.1 & 0.63 & Call et al. 1983 \\
\hline 2-Propanol & Fathead minnow & Acute & 200 & 3160 & 31600 & AQUIRE* \\
\hline Pydrin & Fathead minnow & Chronic & 0.006 & 0.13 & 1.58 & Spehar et al. 1982 \\
\hline 1,1,2,2-Tetrachloroethane & Fathead minnow & Chronic & 251 & 1580 & 50100 & Ahmed et al. 1984 \\
\hline Tetrachloroethene & $\begin{array}{l}\text { Fathead minnow } \\
\text { Bluegill } \\
\text { Rainbow trout }\end{array}$ & $\begin{array}{l}\text { Acute } \\
\text { Acute } \\
\text { Acute }\end{array}$ & $\begin{array}{l}3.16 \\
10 \\
3.16\end{array}$ & $\begin{array}{r}50 \\
100 \\
40\end{array}$ & $\begin{array}{l}398 \\
501 \\
316\end{array}$ & $\begin{array}{l}\text { EPA } 1980 w \\
\text { EPA 1980w } \\
\text { EPA 1980w }\end{array}$ \\
\hline Tetrachloroethylene & Fathead minnow & Chronic & 40 & 1000 & 12600 & Ahmed et al. 1984 \\
\hline Toluene & $\begin{array}{l}\text { Fathead minnow } \\
\text { Bluegill } \\
\text { Guppy }\end{array}$ & $\begin{array}{l}\text { Acute } \\
\text { Acute } \\
\text { Acute }\end{array}$ & $\begin{array}{l}10 \\
13 \\
50\end{array}$ & $\begin{array}{l}126 \\
126 \\
501\end{array}$ & $\begin{array}{l}1000 \\
1000 \\
3160\end{array}$ & $\begin{array}{l}\text { EPA 1980y } \\
\text { EPA 1980y } \\
\text { EPA 1980y }\end{array}$ \\
\hline Toxaphene & Brook trout & Chronic & 0.01 & 0.063 & 0.40 & Mayer et al. 1975 \\
\hline 1,1,1-Trichloroethane & $\begin{array}{l}\text { Fathead minnow } \\
\text { Bluegill }\end{array}$ & $\begin{array}{l}\text { Acute } \\
\text { Acute }\end{array}$ & $\begin{array}{l}18 \\
32\end{array}$ & $\begin{array}{l}200 \\
316\end{array}$ & $\begin{array}{l}1580 \\
1580\end{array}$ & $\begin{array}{l}\text { AQUIRE* } \\
\text { AQUIRE* }\end{array}$ \\
\hline 1,1,2-Trichloroethane & Fathead minnow & Chronic & 1000 & 15800 & 251000 & Ahmed et al. 1984 \\
\hline Trichloroethene & $\begin{array}{l}\text { Fathead minnow } \\
\text { Flagfish } \\
\text { Bluegill }\end{array}$ & $\begin{array}{l}\text { Acute } \\
\text { Acute } \\
\text { Acute }\end{array}$ & $\begin{array}{l}13 \\
20 \\
32\end{array}$ & $\begin{array}{l}158 \\
251 \\
316\end{array}$ & $\begin{array}{l}1260 \\
1260 \\
1990\end{array}$ & $\begin{array}{l}\text { EPA } 1980 z \\
\text { Smith et al. } 1991 \\
\text { EPA } 1980 z\end{array}$ \\
\hline 2-Trifluoromethyl-4-phenol & Brook trout & Chronic & 100 & 501 & 3160 & Dwyer et al. 1978 \\
\hline
\end{tabular}


Table 2 (continued)

\begin{tabular}{llccccc}
\hline \multicolumn{1}{c}{ Chemical } & \multicolumn{1}{c}{ Test species } & $\begin{array}{c}\text { Test } \\
\text { type }\end{array}$ & $\begin{array}{c}\text { Lower } \\
95 \% \mathrm{CL}\end{array}$ & Median & $\begin{array}{c}\text { Upper } \\
95 \% \text { CL }\end{array}$ & Source \\
\hline Trifluralin & Fathead minnow & Chronic & 0.063 & 0.63 & 7.94 & Macek et al. 1976b \\
1,3,5-Trinitrobenzene & Fathead minnow & Chronic & 3.16 & 50 & 631 & Van Der Schalie 1983 \\
& Rainbow trout & Chronic & 13 & 158 & 1580 & Van Der Schalie 1983 \\
& Rainbow trout & Chronic & 13 & 158 & 1580 & Van Der Schalie 1983 \\
& Fathead minnow & Acute & 3.16 & 40 & 398 & ACQUIRE* \\
& Bluegill & Acute & 10 & 126 & 794 & ACQUIRE* $^{*}$ \\
& Guppy & Acute & 25 & 251 & 1580 & ACQUIRE* $^{*}$ \\
\hline
\end{tabular}

*EPA (n.d.) 


\section{CHEMICALSPECIFIC INFORMATION}

This section describes the sources of information and procedures that are specific to individual elements. Except where noted, the sources of data for estimating chronic values and test $\mathrm{EC}_{20}$ s are the same as those used to calculate advisory values (Appendix).

\subsection{INORGANICS}

Ammonia. The test $\mathrm{EC}_{20}$ value for fish is from Thurston et al. (1986).

Aluminum. The toxicity of aluminum has been shown to vary widely with water hardness and $\mathrm{pH}$ (among others are Ingersoll et al., 1990a 1990b; Sadler and Lynam, 1988; and Cleveland et al., 1986). The benchmarks were calculated using only tests in circumneutral water. Lowest chro values for fish are from Kimball (n.d.), and for daphnids from McCauley et al. (1986). Lowest test $\mathrm{EC}_{20}$ values are from Kimball (n.d.).

Antimony. Chronic and test $\mathrm{EC}_{20}$ values for antimony are from Kimball (n.d.).

Arsenic III. Official NAWQC are listed for arsenic III. The lowest chronic values for fish and daphnids are given by Call et al. (1983) and Lima et al. (1984). The test $\mathrm{EC}_{20}$ values are from Lima et al. (1984) for fish and by Call et al. (1983) and Lima et al. (1984) for daphnids.

Arsenic $\mathrm{V}$. The chronic and test $\mathrm{EC}_{20}$ values for fish are from Defoe (1982), and the test $\mathrm{EC}_{20}$ for daphnids is from Spehar et al. (1980). The EPA (1986) gives a lowest observed effect level value in lieu of a NAWQC. This value is the lowest observed effect level for adverse effects on freshwater aquatic plants, and therefore does not correspond to our lowest chronic value.

Beryllium. The chronic and test $\mathrm{EC}_{20}$ values for daphnids are from Kimball (n.d.).

Boron. The test $\mathrm{EC}_{20}$ value for daphnids is from Gerisch (1984).

Cadmium. There are official NAWQC listed for cadmium. The lowest chronic value is from Sauter et al. (1976) for fish and Chapman et al. (n.d.) for daphnids. The test $\mathrm{EC}_{20}$ values are from Carlson et al.(1982) for fish and Elnabarawy et al. (1986) for daphnids.

Chromium III. Official NAWQC are listed for chromium III. The lowest chronic value is from Stevens and Chapman (1984) for tish and by Chapman et al. (n.d.) for daphnids. Stevens and Chapman (1984) also provided data for the test $\mathrm{EC}_{20}$ value for fish.

Chromium VI. There are official NAWQC for chromium VI. The chronic and test $\mathrm{EC}_{20}$ values for fish are from Sauter et al. (1976). For daphnids, the chronic value is from Mount (1982) and the test $\mathrm{EC}_{20}$ from Elnabarawy et al. (1986).

Cobalt. The chronic and test $\mathrm{EC}_{20}$ values for cobalt are from Kimball (n.d.). 
Copper Official NAWQC are listed for copper. The chronic and test $\mathrm{EC}_{20}$ values for fish are from Sauter et al. (1976). The chronic and test $\mathrm{EC}_{20}$ values for daphnids are from Dave (1984a).

Cyanide. There are official NAWQC for cyanide. The chronic and test $\mathrm{EC}_{20}$ values for fish were both from Koenst et al. (1977).

Fluoride ion. Chronic and test $\mathrm{EC}_{20}$ values for daphnids are from Dave (1984b).

Iron. The data for the effects of iron on daphnids was from Dave (1984c).

Lead. There are official NAWQC listed for lead. The lowest chronic value for fish is from Davies et al. (1976) and for daphnids from Chapman et al. (n.d.). The test $\mathrm{EC}_{20}$ value for fish is from Sauter et al. (1976). The acute- $\mathrm{EC}_{20}$ ratio from which the SS test $\mathrm{EC}_{20}$ was calculated had to be obtained using a species mean acute value for Salmo gairdneri (EPA 1985b) since no acute value was reported by Sauter et al.

Manganese. All chronic and test $\mathrm{EC}_{20}$ values for manganese are from Kimball (n.d.).

Mercury, inorganic Mercury has official NAWQC. However, the chronic criterion for mercury is based on the final residue value derived from a methyl mercury bioconcentration factor. For consistency, the criterion used was derived from the final acute and chronic values. The chronic and test $\mathrm{EC}_{20}$ values for fish are from Call et al. (1983), and those for daphnids are from Beisinger et al. (1982). The acute- $\mathrm{EC}_{20}$ ratio used to calculate the daphnid value had to be derived using a species mean acute value (EPA 1985c) as no acute value was reported in Beisinger et al.

Mercury, methyl. The chronic and test $\mathrm{EC}_{20}$ values for fish are from McKim et al. (1976). The chronic and test $\mathrm{EC}_{20}$ values for daphnids are from Beisinger et al. (1982).

Molybdenum. The chronic and test $\mathrm{EC}_{20}$ values for daphnids are from Kimball (n.d.).

Nickel. There are official NAWQC for nickel. However, nickel concentrations of $10 \mathrm{ug} / \mathrm{L}$ in ORR stream water reduced $7 \mathrm{~d}$ Ceriodaphnia dubia survivorship to 60\% (Kszos et al. 1992). This concentration is considerably below the chronic NAWQC for nickel but similar to the lowest of the alternate benchmarks (Table 1). The chronic and test $\mathrm{EC}_{20}$ values for fish are from Nebeker et al. (1985). For daphnids, the chronic value was from Lazareva (1985) and the test $\mathrm{EC}_{20}$ was from Münzinger (1990).

Selenium Official NAWQC are listed for selenium. The chronic and test $\mathrm{EC}_{20}$ values for fish are from Goettl and Davies (1976). The chronic value for daphnids is from Kimball (n.d.), and the test $\mathrm{EC}_{20}$ is from Johnston (1987). The acute- $\mathrm{EC}_{20}$ ratio from which the latter value was calculated had to be derived using a species mean acute value for Daphnia magna (EPA 1987) because no acute value was reported by Johnston.

Silver. There is an official acute NAWQC for silver. The chronic NAWQC was estimated from the FAV and two measured chronic values (Davies et al. 1978, Nebeker et al. 1983). The chronic value for fish is from Davies et al. (1978). The chronic value for daphnids and 
the test $\mathrm{EC}_{20}$ for tish are from Nebeker et al. (1983). The test $\mathrm{EC}_{20}$ for daphnids is from Elnabarawy et al. (1986).

Thallium. All chronic and test $\mathrm{EC}_{20}$ values are from Kimball (n.d.). The EPA (1986) gives a lowest observed effect level value in lieu of a NAWQC. This value is from a document that is unavailable. To be consistent, we derived an advisory value.

Vanadium. The chronic and test $\mathrm{EC}_{20}$ values for fish are from Holdway and Sprague (1979), and for daphnids from Kimball (n.d.).

Zinc. There are official NAWQC for zinc. The chronic and test $\mathrm{EC}_{20}$ values are from Spehar (1976), and the chronic value for daphnids is from Chapman et al. (n.d.).

\subsection{ORGANICS} (1982).

Acenaphthene. The chronic and test $\mathrm{EC}_{20}$ values for fish are from Cairns and Nebeker

Anthracene. The chronic and test $\mathrm{EC}_{20}$ values for daphnids are from Holst and Giesy (1989).

Benzene. The lowest chronic value for daphnids is given by EPA (1978) and the test $\mathrm{EC}_{20}$ value for fish is from Black and Birge (1982). This latter report requires comment: Black and Birge conducted a series of screening tests for a large number of chemicals on several freshwater organisms. Larval fish survival was recorded only to $4 \mathrm{~d}$ posthatch, and LOECs and NOECs were not determined. These tests, then, did not generate chronic values and are not equivalent to the other chronic tests cited in this report. The test $\mathrm{EC}_{20}$ values based on tests by Black and Birge are likely to be high relative to those from conventional chronic tests.

Benzo(a)pyrene. The test $\mathrm{EC}_{20}$ for fish is derived from Hannah et al. (1982).

BHC (lindane). There are official NAWQC listed for lindane. All chronic and test $\mathrm{EC}_{20}$ values are from Macek et al. (1976a). The acute-EC 20 ratio from which the $\mathrm{SS}_{\mathrm{EC}} 20$ was calculated was derived using a species mean acute value for Salvelinus fontinalis (EPA 1980p) since no acute data was reported by Macek et al.

Bis(2-ethylhexyl)phthalate. The chronic and test $\mathrm{EC}_{20}$ values for fish are from Mchrle and Mayer (1976). The chronic and test $\mathrm{EC}_{20}$ values for daphnids are from Mayer and Sanders (1973).

Carbon tetrachloride. The chronic value for fish is from EPA (1978), and the test $\mathrm{EC}_{20}$ value is from Black and Birge (1982). See the note for benzene.

Chlordane. The official chronic NAWQC for chlordane is based on the final residue value. For consistency, we report an estimated criterion derived from the FAV and final acute/chronic ratio. The lowest chronic and test $\mathrm{EC}_{20}$ values are from Cardwell et al. (1977). 
Chloroform. The test $\mathrm{EC}_{20}$ value for fish is from Black and Birge (1982). See the note for benzene. The EPA (1986) gives a lowest observed effect value in lieu of a NAWQC. This value is a $27-\mathrm{d} \mathrm{LC}_{50}$ value, and therefore does not correspond to our lowest chronic value.

DDT. The official chronic NAWQC is based on the final residue value. For consistency, we report an estimated criterion calculated using the FAV and an acute chronic ration derived from the single available chronic value. The chronic and test $\mathrm{EC}_{20}$ values for fish are from Jarvinen et al. (1977). The estimated fish chronic value is from EPA (1980j). (1985).

Di-n-butyl phthalate. All chronic and test $\mathrm{EC}_{20}$ values are from McCarthy and Whitmore

1,2-Dichloroethane. The chronic value for fish is from Ahmed et al. (1984), and the test $\mathrm{EC}_{20}$ value for fish is from Benoit et al. (1982). The chronic and test $\mathrm{EC}_{20}$ values for daphnids are from Richter et al. (1983).

1,1-Dichloroethene. The chronic value for fish is from EPA (1978). (1985).

Di-n-octyl phthalate. All chronic and test $\mathrm{EC}_{20}$ values are from McCarthy and Whitmore

Ethyl benzene. The chronic value for fish is from EPA (1978).

Heptachlor. The chronic NAWQC is based on the final residue value. For consistency, we report an estimated chronic criterion calculated using the FAV and the single available chronic value. The chronic and test $E_{20}$ values for fish are from Macek et al. (1976b). The SS test $\mathrm{EC}_{20}$ value was calculated using an acute-EC $\mathrm{EC}_{20}$ ratio that was derived from a species mean acute value for Pimephales promelas (EPA 1980o) because no acute data is available from Macek et al. (1976b).

4-Methyl-2-pentanone. The chronic value for fish is from Call et al. (1985).

Methylene chloride. The chronic value for fish is from Dill et al. (1987), and the test $\mathrm{EC}_{20}$ value is from Black and Birge (1982). See the note for benzene.

Naphthalene. The chronic and test $\mathrm{EC}_{20}$ values for fish are from DeGraeve et al. (1982), and the test $\mathrm{EC}_{20}$ value for daphnids is from Geiger and Buikema (1982).

4-Nitrophenol. The chronic and test $\mathrm{EC}_{20}$ values for daphnids are from Francis et al. (1986). The EPA (1980r) is the source for the estimated fish chronic value and test $\mathrm{EC}_{20}$.

Nitrophenols (total). In lieu of a NAWQC, EPA (1986) gives an acute value which is the lowest observed effect level and a chronic value which is the lowest observed effect level for a species of algae.

$\mathrm{N}$-nitrosodiphenylamine. The source for the estimated fish and daphnid chronic values and the test $\mathrm{EC}_{20}$ is EPA (1980aa).

PCBs: Aroclor 1242 . The chronic and test $\mathrm{EC}_{20}$ values for fish are from Nebeker et 
al. (1974).

PCBs: Aroclor 1248. The chronic and test $\mathrm{EC}_{20}$ values for tish are from DeFoe et al. (1978), and the chronic and test $\mathrm{EC}_{20}$ values for daphnids are from Nebeker and Puglisi (1974).

PCBs: Aroclor ${ }^{\circ} 1254$. The chronic value for fish is from Mauck et al. (1978), and the test $\mathrm{EC}_{20}$ value is from Nebeker et al. (1974). The chronic and test $\mathrm{EC}_{20}$ values for daphnids are from Nebeker and Puglisi (1974). (1978).

PCBs: Aroclor ${ }^{\circ} 1260$. The chronic and test $\mathrm{EC}_{20}$ values for fish are from DeFoe et al.

Phenanthrene. The chronic and test $\mathrm{EC}_{20}$ values for daphnids are from Geiger and Buikema (1982).

Phenol. The chronic and test $\mathrm{EC}_{20}$ values for fish are from DeGraeve et al. (1980).

1,1,2,2-Tetrachloroethane. The chronic and test $\mathrm{EC}_{20}$ values for fish are from Ahmed et al. (1984), and the values for daphnids are from Richter et al. (1983).

Tetrachloroethene. The chronic and test $\mathrm{EC}_{20}$ values for fish are from Ahmed et al. (1984). The chronic and test $\mathrm{EC}_{20}$ values for daphnids are from Richter et al. (1983). benzene.

Toluene. The test $\mathrm{EC}_{20}$ value for fish is from Black and Birge (1982). See the note for

1,1,1-Trichloroethane. The chronic and test $\mathrm{EC}_{20}$ values for daphnids are from Thompson and Carmichael (1989). EPA 1980h is the source for the estimated fish chronic value and test $\mathrm{EC}_{20}$.

1,1,2-Trichloroethane. The chronic and test $\mathrm{EC}_{20}$ values for fish are from Ahmed et al. (1984), and the chronic and test $\mathrm{EC}_{20}$ values for daphnids are from Richter et al. (1983).

Trichloroethene. The chronic and test $\mathrm{EC}_{20}$ values for fish are from Smith et al. (1991).

Xylene. The test $\mathrm{EC}_{20}$ value for fish is from Black and Birge (1982). See the note for benzene. 


\section{COMPARISON OF BENCHMARKS}

The chronic NAWQC are the lowest benchmark for only $5.5 \%$ of the chemicals for which they have been promulgated (Table 3). This result was expected given the characteristics of the criteria discussed above. At the other extreme, the advisory values using the method of Stephan and Erickson were lowest in every case for which they were calculated (i.e., chemicals with some standard data but no NAWQC). This conservatism was not surprising given the goal of the method of ensuring with $95 \%$ confidence that these values would not exceed the NAWQC. To determine how conservative the advisory values are in practice, we calculated advisory values for the chemicals with official criteria. Because most of the advisory values were calculated using only four or fewer $\mathrm{LC}_{50}$ values (Table 4), we randomly selected one, two, three, and four $\mathrm{LC}_{50}$ s from the lists of eight or more $\mathrm{LC}_{50} \mathrm{~s}$ available for each criterion chemical in Table 1 and calculated advisory values for each data set (Table 5). These results demonstrate that the degree of conservatism required to achieve $95 \%$ confidence is quite large when data are sparse. To indicate the relative conservatism of the advisory values, the number of acute and chronic test data that were used to calculate each value is listed in Table 4.

Table 3. Comparisons of alternative screening benchmarks for aquatic life on the basis of the number of chemicals for which each could be calculated $(n)$, the percentage of those chemicals for which it was the lowest benchmark, and the median, minimum, and maximum ratio of each benchmark to the chronic NAWQC for the criterion chemicals

\begin{tabular}{|c|c|c|c|c|c|}
\hline & \multirow[t]{2}{*}{$\mathbf{n}$} & \multirow{2}{*}{$\begin{array}{l}\text { Percent } \\
\text { lowest }\end{array}$} & \multicolumn{3}{|c|}{$\begin{array}{l}\text { Ratio to chronic } \\
\text { NAWQC }\end{array}$} \\
\hline & & & Median & Minimum & Maximum \\
\hline Chronic NAWQC & 18 & 5.5 & - & & \\
\hline Advisory value & 73 & 100 & - & & \\
\hline $\begin{array}{l}\text { Fish chronic value } \\
\text { Measured } \\
\text { Estimated }\end{array}$ & $\begin{array}{l}44 \\
34\end{array}$ & $\begin{array}{l}4.5 \\
0\end{array}$ & 2.52 & 0.18 & 182.50 \\
\hline $\begin{array}{l}\text { Daphnid chronic val } \\
\text { Measured } \\
\text { Estimated }\end{array}$ & $\begin{array}{l}38 \\
29\end{array}$ & $\begin{array}{l}5.3 \\
0\end{array}$ & 0.74 & 0.02 & 181.25 \\
\hline $\begin{array}{l}\text { Fish test } \mathrm{EC}_{20} \\
\text { Measured } \\
\text { Estimated }\end{array}$ & $\begin{array}{l}47 \\
30\end{array}$ & $\begin{array}{l}2.1 \\
0\end{array}$ & 1.14 & 0.39 & 54.02 \\
\hline Daphnid test $E_{20}$ & 37 & 8.1 & 0.69 & 0.02 & 137.50 \\
\hline$S S$ test $E_{20}$ & 17 & 71 & 0.14 & 0.01 & 2.94 \\
\hline Population $\mathrm{EC}_{20}$ & 68 & () & 1.70 & 0.20 & 28.7 \\
\hline
\end{tabular}


Table 4. Amount of data available and used to calculate advisory values (Advisory values based on more data are less conservative.)

\begin{tabular}{|c|c|c|}
\hline Contaminants & $\begin{array}{c}\text { Number of } \\
\mathrm{LC}_{50}^{\mathrm{s}}\end{array}$ & Number of Chronics \\
\hline Antimony & 2 & 2 \\
\hline Arsenic V & 5 & 1 \\
\hline Barium & 1 & 0 \\
\hline Beryllium & 4 & 1 \\
\hline Cobalt & 2 & 2 \\
\hline Fluorine & 4 & 2 \\
\hline Magnesium & 1 & 0 \\
\hline Manganese & 2 & 1 \\
\hline Mercury, methyl & 2 & 1 \\
\hline Potassium & 1 & 0 \\
\hline Sodium & 1 & 0 \\
\hline Thallium & 3 & 2 \\
\hline Uranium & 1 & 0 \\
\hline Vanadium & 4 & 3 \\
\hline Zirconium & 2 & 0 \\
\hline Acenaphthene & 2 & 1 \\
\hline Acetone & 4 & 0 \\
\hline Anthracene & 1 & 0 \\
\hline Benzene & 4 & 0 \\
\hline Benzidene & 3 & 0 \\
\hline Benzo(a)anthracene & 1 & 0 \\
\hline Benzoic acid & 1 & 0 \\
\hline Benzyl alcohol & 2 & 0 \\
\hline BHC (other than gamma) & 1 & 0 \\
\hline Bis(2-ethylhexyl)phthalate & 4 & 2 \\
\hline 2-Butanone & 3 & 0 \\
\hline Carbon disulfide & 2 & 0 \\
\hline Carbon tetrachloride & 3 & 1 \\
\hline Chlorobenzene & 4 & 0 \\
\hline Chloroform & 3 & 0 \\
\hline p,p'DDD & 7 & 0 \\
\hline Decane & 1 & 0 \\
\hline Di-n-butyl phthalate & 6 & 2 \\
\hline Dibenzofuran & 1 & 0 \\
\hline 1,1-Dichloroethane & 1 & 0 \\
\hline 1,2-Dichloroethane & 3 & 2 \\
\hline 1,1-Dichloroethene & 3 & 0 \\
\hline 1,2-Dichloroethenes & 1 & 0 \\
\hline 1,3-Dichloropropene & 2 & 0 \\
\hline Diethyl phthalate & 2 & 0 \\
\hline Ethyl benzene & 4 & 0 \\
\hline Fluoranthene & 2 & 0 \\
\hline Hexane & 1 & 0 \\
\hline 2-Hexanone & $i$ & 0 \\
\hline 1-Methyl naphthalene & 1 & 0 \\
\hline 4-Methyl-2-pentanone & 1 & 1 \\
\hline
\end{tabular}


Table 4 (continued)

\begin{tabular}{lcc}
\hline \multicolumn{1}{c}{ Contaminants } & $\begin{array}{c}\text { Number of } \\
\text { LC }_{50} \text { s }\end{array}$ & Number of Chronics \\
\hline 2-Methyl phenol & 4 & 0 \\
Methylene chloride & 3 & 1 \\
Naphthalene & 4 & 1 \\
4-Nitrophenol & 5 & 1 \\
N-nitrosodiphenylamine & 2 & 0 \\
3-Octanone & 2 & 0 \\
PCB 1221 & 1 & 0 \\
PCB 1232 & 1 & 0 \\
PCB 1242 & 3 & 2 \\
PCB 1248 & 1 & 1 \\
PCB 1254 & 3 & 1 \\
PCB 1260 & 1 & 0 \\
1-Pentanol & 2 & 0 \\
Phenanthrene & 2 & 0 \\
Phenol & 6 & 2 \\
2-Propanol & 1 & 0 \\
1,1,2,2-Tetrachloroethane & 3 & 2 \\
Tetrachloroethene & 5 & 2 \\
Toluene & 4 & 0 \\
1,1,1-Trichloroethane & 2 & 1 \\
1,1,2-Trichloroethane & 3 & 2 \\
Trichloroethene & 3 & 1 \\
Vinyl acetate & 3 & 0 \\
Vinyl chloride & 1 & 0 \\
Xylene & 2 & 0 \\
\hline
\end{tabular}


Table 5. Quotients of ratios of NAWQC/Advisory Valucs calculated for those chemicals for which NAWQC were available. The advisory values were calculated for each criterion chemical using one, two, three, and four $\mathrm{LC}_{50}$ values chosen at random from the eight or more $\mathrm{LC}_{50}$ s used to calculate the NAWQC for that chemical

(The quotients are a measure of the conservatism of the advisory values.)

\begin{tabular}{|c|c|c|}
\hline Data used & Acute criterion & Chronic criterion \\
\hline $\begin{array}{l}\text { One } \\
\text { LC }_{50} \\
\text { Mean } \\
\text { Minimum } \\
\text { Maximum }\end{array}$ & $\begin{array}{c}1681 \\
4.6 \\
7654\end{array}$ & $\begin{array}{r}19544 \\
52 \\
110,000\end{array}$ \\
\hline $\begin{array}{l}\text { Two } \\
\text { LC }_{50} \\
\text { Mean } \\
\text { Minimum } \\
\text { Maximum }\end{array}$ & $\begin{array}{c}393 \\
6.8 \\
1302\end{array}$ & $\begin{array}{r}3617 \\
77 \\
18333\end{array}$ \\
\hline $\begin{array}{l}\text { Three } \\
\text { LC }_{50^{\mathrm{S}}} \\
\text { Mean } \\
\text { Minimum } \\
\text { Maximum }\end{array}$ & $\begin{array}{r}129 \\
14 \\
367\end{array}$ & $\begin{array}{r}1554 \\
22 \\
4583\end{array}$ \\
\hline $\begin{array}{l}\text { Four } \\
\text { LC }_{50^{\mathrm{s}}} \\
\text { Mean } \\
\text { Minimum } \\
\text { Maximum }\end{array}$ & $\begin{array}{l}6.95 \\
0.04 \\
55\end{array}$ & $\begin{array}{r}44.69 \\
0.42 \\
239\end{array}$ \\
\hline
\end{tabular}

The other potential lower screening benchmarks are intermediate in their conservatism. In general, benchmarks based on effects on fish are less conservative than those based on daphnids or than the NAWQC that include daphnids. This result is consistent with other studies (Stephan and Erickson n.d., Suter 1992).

The test $\mathrm{EC}_{20}$ values are lower than the CVs for the same taxa in $62.5 \%$ and $82 \%$ of cases for fish and daphnids, respectively. This result is expected given that the test $\mathrm{EC}_{20} \mathrm{~s}$ integrate across life stages but the CVs do not.

After the advisory values, the most conservative benchmark is the SS test $\mathrm{EC}_{20}$. These values are on average $14 \%$ of the NAWQC when both could be calculated. They are equivalent to the NAWQC but use integrative test endpoints.

The fact that some CVs for fish and daphnids are lower than chronic NAWQC is surprising at first because conceptually the chronic NAWQC are estimates of CVs for sensitive species. However, the CVs are not used directly but rather are used to calculate acute/chronic ratios that are used in the criteria calculation. Unless a species that is highly 
sensitive in a chronic test has a high acute/chronic ratio, it will not lower the chronic NAWQC.

The relative utility of the benchmarks is determined in large part by their availability. The NAWQC and the SS test $\mathrm{EC}_{20}$ values are available for relatively few of the chemicals detected on the ORR because they require large data sets. The Advisory Values and the population $\mathrm{EC}_{20}$ are available for most of the chemicals because they each require only a single $\mathrm{LC}_{50}$. For the same reason, if estimated values are included, the $\mathrm{CVs}$ for fish and daphnids and the test $\mathrm{EC}_{20}$ s for fish are available for most chemicals.

The measured test $\mathrm{EC}_{20}$ values differ from the other potential benchmarks in that they represent an observed and specified effect. All other benchmarks are estimates (population $\mathrm{EC}_{20} \mathrm{~s}$ and estimated CVs and test $\mathrm{EC}_{20} \mathrm{~s}$ ), correspond to no particular effect (CVs and NAWQC), or both (Advisory Values).

Finally, the Advisory Values differ from the other benchmarks in that they are based on lower $5 \%$ confidence bounds rather than best estimates of a threshold for significant effects. The lower $5 \%$ confidence bound on the Population $\mathrm{EC}_{20}$ could $\mathrm{hr}$ used as an equivalently conservative benchmark (Table 2). Lower $5 \%$ prediction intervals can be derived for the estimated $\mathrm{CV}_{\mathrm{s}}$ and test $\mathrm{EC}_{20}$ s by subtracting the PIs listed in the text from the base 10 logs of the estimates reported in Table 1. (For all three of these equations, the variance changes negligibly with deviation from mean X.) These lower likelyhood bounds were not used because the other benchmarks do not have the legal imperative of the NAWQC. However, to be certain of a conservative screen, lower bounds could be substituted for the population $\mathrm{EC}_{20} \mathrm{~s}$ and estimated $\mathrm{CVs}$ and test $\mathrm{EC}_{20}$ s listed in Table 1. 


\section{APPLICATION OF BENCHMARKS}

Each of the alternative benchmarks has a different interpretation. Exceedances of NAWQC create a regulatory imperative for action under CERCLA because they are ARARS. Exceedance of an advisory value implies a greater than $5 \%$ chance that the NAWQC, if its value were known, would be exceeded. Exceedance of a CV indicates that the field concentration is greater than a concentration dividing statistically insignificant from significant effects in a chronic toxicity test. Exceedance of a test $\mathrm{EC}_{20}$ indicates that biologically significant effects levels were exceeded in a chronic toxicity test. Exceedance of the SS test $\mathrm{EC}_{20}$ indicates that a biologically significant effect level may be exceeded in a sensitive species. Exceedance of a population $\mathrm{EC}_{20}$ indicates that a significant reduction in a largemouth bass population could occur. Hence, exeedence of either the acute or chronic NAWQC indicates a need for action. Exceedance of a chronic advisory level implies a low risk. Exceedance of any of the other benchmarks indicates a risk of real effects, which should lead to additional data collection and assessment. However, these inferences all depend on comparison of the benchmarks to appropriate water concentrations.

As discussed in the introduction, the chronic benchmarks are to be used as lower screening benchmarks. The acute NAWQC and advisory values are to be used as upper screening benchmarks. However, because of their conservatism, exceedance of the acute advisory values cannot be taken to incicate that severe effects are likely to be occurring. If an acute advisory value is exceeded, the assessor should examine the acute values used to generate the advisory value (Appendix A) and judge whether in fact severe effects are likely.

All of these benchmarks are based on toxicity tests conducted in the laboratory. Therefore, they should be compared to water concentrations that are as equivalent as possible to concentrations in test water that is nearly all dissolved. That is, they should be compared to concentrations dissolved in ambient water |i.e., 0.1-0.45 um filtered before being extracted with acid or organic solvents (HECD 1992)]. Because total recoverable concentrations are required for human health risk assessments, they, rather than dissolved concentrations, are often reported. However. for metals, total recoverable concentrations typically include $30-95 \%$ particle-bound material (HECD 1992). Therefore, total recoverable concentrations should be used for aquatic ecological risk assessments only if dissolved concentrations are not available.

The NAWQC for hardness-dependent metals are based on a hardness of $100 \mathrm{mg} / \mathrm{L}$, which is appropriate for ambient waters on the ORR. If these benchmarks are applied to a site with hard or soft water, the NAWQC for those metals should be recalculated as recommended by the EPA.

These benchmarks may also be applied to sediments if pore water concentrations can be measured or estimated. The EPA (OWRS 1989) has recommended that sediment concentrations be converted to pore water concentrations with equilibrium partitioning models so that the NAWQC can be used as sediment quality criteria. Guidance for assessment of ecological risks from sediments will be presented in a separate document. 


\section{REFERENCES}

Adelman, I. R., L. L. Smith, and G. D. Siesennop. 1976. Chronic toxicity of Guthion to the fathead minnow (Pimephales promelas Rafinesque). Bull. Environ. Contam. Toxicol. 15:726-733.

Ahmed, N., D. Benoit, L. Brook, D. Call, A. Carlson, D. DeFoe, H. Huot, A. Moriarity, J. Richter, P. Shubat, G. Veith, and C. Wallbridge. 1984. Aquatic toxicity tests to characterize the hazard of volatile organic chemicals in water: A toxicity data summary-Parts I and II, EPA-600/3-84-009. U.S. Environmental Protection Agency, Duluth, Minn.

Allison, D. T. 1977. Use of exposure units for estimating aquatic toxicity of organophosphate pesticides, EPA-600/3-77-077. U.S. Environmental Protection Agency, Duluth, Minn.

Allison, D. T., and R. O. Hermanutz. 1977. Toxicity of diazinon to brook trout and fathead minnows, EPA-600/3-77-060. U.S. Environmental Protection Agency, Duluth, Minn.

Arthur, J. W., and J. G. Eaton. 1971. Chloramine toxicity to the amphipod Gammarus pseudolimnaeus and the fathead minnow (Pimephales promelas). J. Fish. Res. Board Canada. 28:1841-1845.

Barnthouse, L.W., G.W. Suter II, and A. E. Rosen. 1990). Risks of toxic contaminants to exploited fish populations: influence of life history, data uncertainty and exploitation intensity. Environ. Toxicol. Chem. 9:297-311.

Bartell, S. M. 1990. Ecosystem context for estimating stress-induced reductions in fish populations. pp. 167-182. IN American Fisheries Society Special Symposium 8. Bethesda, MD.

Beisinger, K. E., L. E. Anderson, and J. G. Eaton. 1982. Chronic effects of inorganic and organic mercury on Daphnia magna: toxicity, accumulation, and loss. Arch. Environ. Contam. Toxicol. 11:769-774.

Benoit, D. A. 1975. Chronic effects of copper on survival, growth, and reproduction of the bluegill (Lepomis macrochirus). Trans. Am. Fish. Soc. 104:353-358.

Benoit, D. A., and G. W. Holcombe. 1978. Toxic effects of zinc on fathead minnows Pimephales promelas in soft water. J. Fish Biol. 13:701-708.

Benoit, D. A., En. N. Leonard, G. M. Christensen, and J. T. Fiandt. 1976. Toxic effects of cadmium on three generations of brook trout (Salvelinus fontinaiis). Trans. Amer. Fish. Soc. 105:550-560.

Benoit, D. A., F. A. Puglisi, and D. L. Olson. 1982. A lathead minnow Pimephales promelas early life ctage toxicity test method evaluation and exposure to four organic chemicals. Environ. Pollut. (Ser. A) 28:189-197.

Beusen, J.-M., and B. Neven. 1987. Toxicity of vanadium to different freshwater organisms. Bull. Environ. Contam. Toxicol. 39:194-201.

Black. J. A., and W. J. Birge. 1982. The aquatic toxicity of organic compounds to embryo-larval stages of fish and amphibians. University of Kentucky, Water Resources Research Institute, Lexington, Kentucky.

Brungs, W. A. 1969. Chronic toxicity of zinc to the fathead minnow Pimephales promelas Rafinesque. Trans. Am. Fish. Soc. 98:272-279.

Cairns, M. A., and A. V. Nebeker. 1982. Toxicity ofacenaphthene and isophorone to early life stages of fathead minnows. Arch. Environ. Contam. Toxicol. 11:703-707. 
Call, D. J., L. T. Brook, N. Ahmed, and J. E. Richter. 1983. Toxicity and metabolism studies with EPA priority pollutants and related chemicals in freshwater organisms, EPA-600/3-83-095. U.S. Environmental Protection Agency, Duluth, Minn.

Call, D. J., L. T. Brook, M.L. Knuth, S. H. Poirer, and M.D. Hoglund. 1985. Fish subchronic toxicity prediction model for industrial organic chemicals the produce narcosis. Environ. Toxic. and Chem. 4:335-341.

Cardwell, R. D., D. G. Foreman, T. R. Payne, and D. J. Wilbur. 1977. Acute and chronic toxicity of chlordane to fish and invertebrates, EPA-600/3-77-019. U.S. Environmental Protection Agency, Duluth, Minn.

Carison, A. R. 1971. Effects of long-term exposure to carbaryl (sevin) on survival, growth, and reproduction of the fathead minnow (Pimephales promelas). J. Fish. Res. Board Can. 29:583-587.

Carlson, A. R., J. A. Tucker, V. R. Mattson, G. L. Phipps, P. M. Cook, G. F. Olson, and F. A. Puglisi. 1982. Cadmium and endrin toxicity to fish in waters containing mineral fibers, EPA-600/3-82-053. U.S. Environmental Protection Agency, Duluth, Minn.

Chapman, G. A., S. Ota, F. Recht. n.d. Effects of water hardness on the toxicity of metals to Daphnia magna. U.S. Environmental Protection Agency, Cornvallis, Oregon.

Cleveland, L., E. E. Little, S. J. Hamilton, D. R. Buckler, and J. B. Hunn. 1986. Interactive toxicity of aluminum and acidity to early life stages of brook trout. Trans. Amer. Fish. Soc. 115:610-620.

Cushman, R. M., S. G. Hildebrand, R. H. Strand, and R. M. Anderson. 1977. The toxicity of 35 trace elements in coal to freshwater biota: a data base with automated retrieval capabilities. ORNL/TM-5793. Oak Ridge National Laboratory.

Dave, G. 1984a. Effects of copper on growth, reproduction, survival and haemoglobin in Daphnia magna. Comp. Biochem. Physiol. 78C:439-443.

Dave, G. 1984b. Effects of fluoride on growth, reproduction, and survival in Daphnia magna. Comp. Biochem. Physiol. 78C:425-431.

Dave, G. 1984c. Effects of waterborne iron on growth, reproduction, survival and haemoglobin in Daphnia magna. Comp. Biochem. Physiol. 78C:433-438.

Davies, P. H., J. P. Goettl, Jr., J. R. Sinley. 1978. Toxicity of silver to rainbow trout (Salmo gairdneri). Water Res. 12:113-117.

Davies, P. H., J. P. Goettl, Jr., J. R. Sinley and N. F. Smith. 1976. Acute and chronic toxicity of lead to rainbow trout Salmo gairdneri, in hard and soft water. Water Res. 10:199-206.

DeFoe, D. L, 1982. Memorandum to Robert L. Spehar. U.S. Environmental Protection Agency, Duluth, Minn. July 9.

DeFoe, D. L., G. D. Veith, and R. L. Carlson. 1978. Effects of Aroclor 1248 and 1260 on the fathead minnow (Pimephales promelas). J. Fish. Res. Board Can. 7:997-1002.

DeGraeve, G. M., R. G. Elder, D. C. Woods, and H. L. Bergman. 1982. Effects of naphthalene and benzene on fathead minnows and rainbow trout. Arch. Environ. Contam. Toxicol. 11:478-490.

DeGraeve, G. M., D. L. Geiger, J. S. Meyer, and H. L. Bergman. 1980. Acute and embryo-larval toxicity of phenolic compounds to aquatic biota. Arch. Environ. Contam. Toxicol. 9:557-568.

Dill, D. C., P. G. Murphy, and M. A. Mayes. 1987. Toxicity of methylene chloride to life stages of the fathead minnow, Pimephales promelas Rafinesque. Bull. Environ. Contam. Toxicol. 39:869-876.

DiToro, D. M. 1985. A particle interaction model of reversible organic chemical sorption. Chemosphere 14:1503-1538. 
Dominguez, S. E. and G. A. Chapman. 1984. Effect of pentachlorophenol on the growth and mortality of embryonic and juvenile steelhead trout. Arch. Environ. Contam. Toxicol. 13:739-743.

Dwyer, W. P., F. L. Mayer, J. L. Allen, and D. R. Buckler. 1978. Chronic and simulated use-pattern exposures of brook trout (Salvelinus fontinalis) to 3-trifluoromethyl-4nitrophenol (TFM). Investigations in Fish Control. 84:1-6.

Eaton, J. G. 1974. Chronic cadmium toxicity to the bluegill (Lepomis macrochirus Rafinesque). Trans. Am. Fish. Soc. 103:729-735.

Elnabarawy, M. T., A. N. Welter, and R. R. Robideau. 1986. Relative sensitivity of three daphnid species to selected organic and inorganic chemicals. Environ. Toxicol. Chem. 5:393-398.

EPA. (n.d.). Aquire: Aquatic Information Retrieval Toxicity Data Base. National Technical Information Service, Springfield, Va.

EPA. 1978. In-depth studies on health and environmental impacts of selected water pollutants. Contract No. 68-01-4646. U.S. Environmental Protection Agency, Washington, D.C. EPA. 1980a. Ambient water quality criteria for acenaphthene, EPA 440/5-80-015. U.S. Environmental Protection Agency, Washington, D.C.

EPA. 1980a. Ambient water quality criteria for antimony, EPA 440/5-80-(020. U.S. Environmental Protection Agency, Washington, D.C.

EPA. 1980b. Ambient water quality criteria for benzidene, EPA 440/5-80-023. U.S. Environmental Protection Agency, Washington, D.C.

EPA. 1980c. Ambient water quality criteria for benzene, EPA 440/5-80-018. U.S. Environmental Protection Agency, Washington, D.C.

EPA. 1980d. Ambient water quality criteria for benzidene, EPA 440/5-80-023. U.S. Environmental Protection Agency, Washington, D.C.

EPA. 1980e. Ambient water quality criteria for beryllium, EPA 440/5-80-024. U.S. Environmental Protection Agency, Washington, D.C.

EPA. 1980f. Ambient water quality criteria for carbon tetrachloride, EPA 440/5-80-(026. U.S. Environmental Protection Agency, Washington, D.C.

EPA. 1980g. Ambient water quality criteria for chlorinated benzenes, EPA 440/5-80-029. U.S. Environmental Protection Agency, Washington, D.C.

EPA. 1980h. Ambient water quality criteria for chlorinated ethanes, EPA 440/5-80-029. U.S. Environmental Protection Agency, Washington, D.C.

EPA. 1980i. Ambient water quality criteria for chloroform, EPA 440/5-80-033. U.S. Environmental Protection Agency, Washington, D.C.

EPA. 1980j. Ambient water quality criteria for DDT, EPA 44(1/5-80-038. U.S. Environmental Protection Agency, Washington, D.C.

EPA. 1980k. Ambient water quality criteria for dichloroethylenes, EPA 440/5-80-041. U.S. Environmental Protection Agency, Washington, D.C.

EPA. 19801. Ambient water quality criteria for dichloropropane and dichloropropene, EPA 440/5-80-043. U.S. Environmental Protection Agency, Washington, D.C.

EPA. 1980m. Ambient water quality criteria for ethylbenzene, EPA 440/5-80-048. U.S. Environmental Protection Agency, Washington, D.C.

EPA. 1980n. Ambient water quality criteria for fluoranthene, EPA 440/5-80-(049. U.S. Environmental Protection Agency, Washington, D.C.

EPA. 1980o. Ambient water quality criteria for heptachlor, EPA 440/5-80-052. U.S. Environmental Protection Agency, Washington, D.C.

EPA. 1980p. Ambient water quality criteria for hexachlorocyclohexane, EPA 440/5-80-054. U.S. Environmental Protection Agency, Washington, D.C. 
EPA. 1980q. Ambient water quality criteria for naphthalene, EPA 440/5-80-059. U.S. Environmental Protection Agency, Washington, D.C.

EPA. 1980r. Ambient water quality criteria for nitrophenols, EPA 440/5-80-(063. U.S. Environmental Protection Agency, Washington, D.C.

EPA. 1980s. Ambient water quality criteria for phenol, EPA 440/5-80-()66. U.S. Environmental Protection Agency, Washington, D.C.

EPA. 1980t. Ambient water quality criteria for polychlorinated biphenyls, EPA 440/5-80-()68. U.S. Environmental Protection Agency, Washington, D.C.

EPA. 1980u. Ambient water quality criteria for silver. EPA 440/5-80-071. U.S. Environmental Protection Agency, Washington, D.C.

EPA. 1980v. Ambient water quality criteria for beryllium. NTIS, Springfield, Virginia.

EPA. 1980w. Ambient water quality criteria for tetrachloroethylene, EPA 440/5-80-073. U.S. Environmental Protection Agency, Washington, D.C.

EPA 1980x. Ambient water quality criteria for thallium, EPA 440/5-80-074. U.S. Environmental Protection Agency, Washington, D.C.

EPA. 1980y. Ambient water quality criteria for toluene, EPA 440/5-80-(075. U.S. Environmental Protection Agency, Washington, D.C.

EPA. 1980z. Ambient water quality criteria for trichloroethylene, EPA 440/5-8()-(077. U.S. Environmental Protection Agency, Washington, D.C.

EPA. 1980aa. Ambient water quality criteria for nitrosamines, EPA 440/5-80-(064. U.S. Environmental Protection Agency, Washington, D.C.

EPA. 1985a. Ambient water quality criteria for arsenic-1984, EPA 440/5-84-033. U.S. Environmental Protection Agency, Washington, D.C.

EPA. 1985b. Ambient water quality criteria for kead-1984, EPA 440/5-84-027. U.S. Environmental Protection Agency, Washington, D.C.

EPA. 1985c. Ambient water quality criteria for mercury-1984, EPA 440/5-84-(126. U.S. Environmental Protection Agency, Washington, D.C.

EPA. 1986. Quality criteria for water-U.S. Environmental Protection Agency, Washington, D.C. 1986, EPA 440/5-86-(0)1.

EPA. 1987. Ambient water quality criteria for selenium, 1987, EPA 440/5-87-(0)6. U.S. Environmental Protection Agency, Washington, D.C.

Ernst, W. R., and E. T. Garside. 1987. Lethal effects of vanadium to two life stages of brook trout Salvclinus fontinalis (Mitchill). Can. J. Zool. 65:628-6.34.

Fieser, A. H., J. L. Sykora, M. S. Kostalos, Y. C. Wu, and D. W. Weycl. 1986. Effect of fluorides on survival and reproduction of Daphnia magna. J. Water Pollut. Control Fed. $58(1): 82-86$.

Finlayson, B. J., and K. M. Verrue. 1980). Estimated safe zinc and copper levels for chinook salmon, Oncorhyncus tshawytscha, in the upper Sacramenton River, California. Calif. Fish and Game. 66(2):68-82.

Finlayson, B. J., and K. M. Verrue. 1985. Toxicities of hutoxyethanol ester and propylene glycol butyl ether ester formulations of 2,4-dichlorophenoxy acetic acid (2,4-D) to juvenile salmonids. Arch. Environ. Contam. Toxicol. 14:153-160.

Francis, P. C., D. W. Grothe, and J. C. Scheuring. 1986. Chronic toxicity of 4-nitrophenol to Daphnia magna Straus under static-renewal and hlow-through conditions. Bull. Environ. Contam. Toxicol. 36:73()-737

Geiger, J. G., and A. L. Buikema Jr. 1982. Hydrociarbons depress growth and reproduction of Daphnia pulex (Clodocera). Can. J. Fish. Aquat. Sci. 39:830-836.

Gerisch, F. M. 1984. Evaluation of static renewal chronic toxicity test method for Daphnia magna Straus using boric acid. Environ. Toxicol. Chem. 3:89-94. 
Goettl, J. P., Jr., and P. H. Davies. 1976. Water pollution studies. Federal Aid Project F-33-R-11. Department of Natural Resources, Colorado Division of Wildlife.

Hamelink, J. L., D. R. Buckler, I. L. Mayer, D. U. Palawski, and H. O. Sanders. 1986. Toxicity of fluridone to aquatic invertebrates and fish. Environ. Toxicol. Chem. 5:97-94.

Hannah, J. B., J. E. Hose, M. L. Landolt, B. S. Miller, S. P. Felton, and W. T. Iwaoko. 1982. Benzo(a)pyrene-induced morphologic and developmental abnormalities in rainbow trout. Arch. Environ. Contam. Toxicol. 11:727-734.

Hazel, C. R. and S. J. Meith. 1970. Bioassay of king slamon eggs and sac fry in copper solutions. Calif. Fish Game. 2:121-124.

HECD (Health and Criteria Division). 1992. Interim guidance on interpretation and implementation of aquatic life criteria for metals. U.S. Environmental Protection Agency, Washington, D.C.

Hermanutz, R. O. 1978. Endrin and malation toxicity to flagfish (Jordanella floridae). Arch. Environ. Contam. Toxicol. 7:159-168.

Hermanutz, R. O., R. H. Mueller, and K. D. Kempfer. 1973. Captan toxicity to fathead minnows (Pimephales promelas), bluegills(Lepomis macrochirus), and brook trout (Salvelinus fontinalis). J. Fish. Res. Board Can. 30:1811-1817.

Holcombe, G. W., D. A. Benoit, E. N. Leonard, and J. M. McKim. 1976. Long term effects of lead exposure on three generations of brook trout (Salvelinus fontinalis). J. Fish. Res. Board Can. 33:1731-1741.

Holcombe, G. W., G. L. Phipps, and J. T. Fiandt. 1982. Effects of phenol, 2,4-dimethylphenol, 2,4-dichlorophenol, and pentachlorophenol on embryo, larval, and early-juvenile fathead minnows (Pimephales promelas). Arch. Environ. Contam. Toxicol. 11(1):73-78.

Holcombe, G. W., G. L. Phipps, and J. T. Fiandt. 1983. Toxicity of selected priority pollutants to various aquatic organisms. Ecotoxicol. Environ. Saf. 740()-409.

Holdway, D. A., and J. B. Sprague. 1979. Chronic toxicity of vanadium to flaglish. Water Res. 13:905-910.

Holman, W. F., and K. J. Macek. 1980. An aquatic safety assessment of linear alkylbenzsulfonate (LAS): chronic effects on fathead minnows. Trans. Am. Fish. Soc. 109:122-131.

Holst, L. L., and J. P. Giesy. 1989. Chronic effects of the photoenhaneed toxicity of anthracene on Daphnia magna reproduction. Environ. Toxicol. Chem. 8:933-942.

Ingersoll, C. G., D. D. Gulley, D. R. Mount, M. E. Mueller, J. D. Fernandez, J. R. Hockett, and H. L. Bergman. 1990)a. Aluminum and acid toxicity to two strains of brook trout (Salvelinus fontinalis). Can. J. Fish. Aquat. Sci. 47:1641-1648.

Ingersoll, C. G., D. R. Mount, D. D. Gulley, T. W. La Point, and H. L. Bergman. 1990b. Effects of $\mathrm{pH}$, aluminum, and calcium on survival and growth of eggs and fry of brook trout (Salvelinus fontinalis). Can. J. Fish. Aquat. Sci. 47:158()-1592.

International Atomic Energy Agency. 1982. Generic models and parameters for assessing the environmental transfer of radionuclides from routine releases. Safety Series No. 57. Vienna.

Jarvinen, A. W., M. J. Hoffman, and T. W. Thorslund. 1977. Long-term toxic effects of DDT food and water exposure on fathead minnows (Pimephales promelas). J. Fish. Res. Board Can. 34:2089-2103.

Jarvinen, A. W., B. R. Nordling, and M. E. Henry. 1983. Chronic toxicity of durshan (chlorpyrifos) to the fathead minnow (Pimephales promelas) and the resultant acetycholinesterease inhibition. Ecotoxicol. Environ. Salety. 7:423-4.34. 
Johnston, P. A. 1987. Acute toxicity of inorganic selenium to Daphnia magna (Straus) and the effect of sub-acute exposure upon growth and reproduction. Aquat. Toxicol. 10:335-352.

Kimball, G. n.d. The effects of lesser known metals and one organic to fathead minnows [Pimephales promelas] and Daphnia magna. U.S. Environmental Protection Agency, Duluth, MN.

Kleiner, C. F., R. L. Anderson, and D. K. Tanner. 1984. Toxicity of fenitrothion to fathead minnows (Pimephales promelas) and alternate exposure duration studies with fenitrothion and endosulfan. Arch. Enivron. Contam. Toxicol. 13:573-578.

Knowles, C. O., M. J. McKee, and D. U. Palawski. 1987. Chronic effects of di-2-ethylhexyl phthalate on biochemical composition, survival and reproduction of Daphnia magna. Environ. Toxicol. Chem. 6:201-208.

Koenst, W. M., L. L. Smith, Jr., and S. J. Broderius. 1977. Effect of chronic exposure of brook trout to sublethal concentrations of hydrogen cyanide. Environmental Science and Technology 11:883-886.

Kszos, L. A., A. J. Stewart, and P. A. Taylor. 1992. An evaluation of nickel toxicity to Ceriodaphnia dubia and Daphnia magna in a contaminated stream and in laboratory tests. Environ. Toxicol. Chem. 11:10)1-1012.

Lazareva, L. P. 1985. Changes in biological characteristics of Daphnia magna from chronic action of copper and nickel at low concentrations. Hydrobiol. J. 21(5):59-62.

LeBlanc, G. A., J. D. Mastone, A. P. Paradice, B. F. Wilson, H. B. Lockhart, Jr., and K. A. Robillard. 1984. The influence of speciation on the toxicity of silver to fathead minnow (Pimephales promelas). Environ. Toxicol. Chem. 3:37-46.

Leduc, G. 1978. Deleterious effects of cyanide on early life stages of atlantic salmon (Salmo salar). J. Fish. Res. Board Can. 35:166-174.

Lima, A. R., C. Curtis, D. E. Hammermeister, T. P. Markee, C. E. Northcott, and L. T. Brooke. 1984. Acute and chronic toxicities of arsenic(III) to fathead minnows, flagfish, daphnids, and an amphipod. Arch. Environ. Contam. Toxicol. 13:595-601.

Lind, D., K. Alto, and S. Chatterton. 1978. Regional copper-nickel study; aquatic toxicology study. Minnesota Environmental Quality Board.

Macek, K. J., K. S. Buxton, S. K. Derr, J. W. Dean, and S. Sauter. 1976a. Chronic toxicity of lindane to selected aquatic invertebrates and fish. EPA-600/3-76-(046. U.S. Environmental Protection Agency, Duluth, Minn.

Macek, K. J., M. A. Lindberg, S. Sauter, K. Buxton, and P. A. Costa. 1976b. Toxicity of four pesticides to water fleas and fathead minnows, EPA-600/3-76-099. U.S. Environmental Protection Agency, Duluth, Minn.

Mauck, W. L., P. M. Mehrle, and F. L. Mayer. 1978. Elfects of the polychlorinated biphenyl Aroclor 1254 on growth, survival, and bone development in brook trout (Salvelinus fontinalis). J. Fish. Res. Board Can. 35:1084-1088.

Mayer, F. L., Jr., P. M. Mehrle, Jr., and W. P. Dwyer. 1975. Toxaphene effects on reproduction, growth and mortality of brook trout, EPA-60)/3-75-(013. U.S. Environmental Protection Agency, Duluth, Minn.

Mayer, F. L., Jr., and H. O. Sanders. 1973. Toxicology of phthalic acid esters in aquatic organisms. Environmental Health Perspectives 5:153-157.

Mayes, M. A., H. C. Alexander, D. L. Hopkins, P. B. Latvaitis. 1986. Acute and chronic toxicity of ammonia to freshwater fish: a site-specific study. Environ. Toxicol. Chem. $5: 437-442$. 
McCarthy, J. F., and D. K. Whitmore. 1985. Chronic toxicity of di-n-butyl and di-n-octyl phthalate to Daphnia magna and the fathead minnow. Environ. Toxicol. Chem. 4:167-179.

McCauley, D. J., L. T. Brooke, D. J. Call, and C. A. Lindberg. 1986. Acute and chronic toxicity of aluminum to Ceriodaphnia duhia at various pH's. Center for Lake Superior Environmental Studies, University of Wisconsin-Superior, Superior, Wis.

McKim, J. M., and D. A. Benoit. 1971. Effects of long-term exposures to copper on survival, growth, and reproduction of brook trout (Salvelinus fontinalis). J. Fish. Res. Board Can. 28:655-662.

McKim, J. M., G. F. Olson, G. W. Holcombe, and E. P. Hunt. 1976. Long-term effects of methylmercuric chloride on three generations of brook trout (Salvelinus fontinalis): toxicity, accumulation, distribution, and elimination. J. Fish. Res. Board Can. 33:2726-2739.

Mehrle, P. M., and F. L. Mayer. 1976. Di-2-ethylhexyl phthalate: residue dynamics and biological effects in rainbow trout and fathead minnows. Trace Subst. Env. Health 10:519-524.

Mount, D. I. 1968. Chronic toxicity of copper to fathead minnows (Pimephales promelas Rafinesque). Water Res. 2:215-223.

Mount, D. I. 1982. Memorandum to Charles E. Spehar. U.S. Environmental Protection Agency, Duluth, Minn. June 7.

Mount, D. I., and C. E. Stephan. 1969. Chronic toxicity of copper to the fathead minnow (Pimephales promelas) in soft water. J. Fish. Res. Board Can. 26:2449-2457.

Münzinger, A. 1990. Effects of nickel on Daphnia magna during chronic exposure and alterations in the toxicity to generations pre-exposed to nickel. Wat. Res. 24:845-852.

Nebeker, A. V., C. K. McAuliffe, R. Mshar, and D. G. Stevens. 1983. Toxicity of silver to steelhead and rainbow trout, fathead minnows, and Daphnia magna. Environ. Toxicol. Chem. 2:95-104.

Nebeker, A. V., C. Savonen, and D. G. Stevens. 1985. Sensitivity of rainbow trout early life stages to nickel chloride. Environ. Toxicol. Chem. 4:233-239.

Nebeker, A. V., and F. A. Puglisi. 1974. Effect of polychlorinated biphenyl (PCB's) on survival and reproduction of Daphnia, Gammarus, and Tanytarsus. Trans. Amer. Fish. Soc. 103:722-728.

Nebeker, A. V., F. A. Puglisi, and D. L. DeFoe. 1974. Effect of polychlorinated biphenyl compounds on survival and reproduction of the fathead minnow and flagfish. Trans. Amer. Fish. Soc. 103:562-568.

OWRS (Office of Water Regulations and Standards). 1986. Quality criteria for water, 1986. EPA 550/5-86-001. Washington, D.C.

OWRS (Office of Water Regulations and Standards). 1989. Briefing report to the EPA Science Advisory Board on the equilibrium partitioning approach to generating sediment quality criteria. EPA 440/5-89-002. Washington, D.C.

OWRS \& ORD (Office of Water Regulations and Standards and Office of Research and Development). 1987. Guidelines for deriving ambient aquatic life advisory concentrations. U.S. Environmental Protection Agency, Washington, D.C.

Pickering, Q. H. 1974. Chronic toxicity of nickel to the fathead minnow and flagfish. Trans. Am. Fish. Soc. 103:562-568.

Pickering, Q. H. 1980. Chronic toxicity of hexavalent chromium to the fathead minnow (Pimephales promelas). Arch. Environ. Contam. Toxicol. 9:405-413.

Pickering, Q. H., and M. H. Gast. 1972. Acute and chronic toxicity of cadmium to the fathead minnow (Pimephales promelas). J. Fish. Res. Board Can. 29:1099-1106. 
Pickering, Q. H., and W. T. Gilliam. 1982. Toxicity of aldicarb and fonofos to the early life-stage of the fathead minnow. Arch. Environ. Conatm. Toxicol. 11:699-702.

Pickering, Q. H., and T. O. Thatcher. 1970. The chronic toxicity of linear allcylate sulfonate (LAS) to Pimephales promelas, Rafinesque. J. Water Pollut. Control Fed. 42:243-254.

Richter, J.E., S. F. Peterson, and C. F. Kleiner. 1983. Acute and chronic toxicity of some chlorinated benzenes, chlorinated ethanes, and tetrachlorocthylene to Daphnia magna. Arch. Environ. Contam. Toxicol. 12:679-684.

Sadler, K., and S. Lynam. 1988. The intluence of calcium on aluminum-induced changes in the growth rate and mortality of brown trout, Salmo trutta L. J. Fish Biol. 33:171-179.

Sauter, S., K. S. Buxton, K. J. Macek, and S. R. Petrocelli. 1976. Effects exposure to heavy metals on selected freshwater fish, EPA-600/3-76-105. U.S. Environmental Protection Agency, Duluth, Minn.

Seim, W. K., L. R. Curtis, S. W. Blenn, and G. A. Chapman. 1984. Growth and survival of developing steelhead trout (Salmo gairdneri) continuously or intermittently exposed to copper. Can. J. Fish. Aquat. Sci. 41:433-438.

Sinley, J. R., J. P. Goettl, Jr., and P. H. Davies. 1974. The effects of zinc on rainbow trout (Salmo gairdneri) in hard and soft water. Bull. Environ. Contam. Toxicol. 12:193-201.

Smith, A. D., A. Bharath, C. Mallard, D. Orr, K. Smith, J. A. Sutton, J. Vukmanich, L. S. McCarty, and G. W. Ozburn. 1991. The acute and chronic toxicity of ten chlorinated organic compounds to the American Flagfish (Jordanella floridae). Arch. Environ. Contam. Toxicol. 20:94-102.

Smith, L. L., S. J. Broderius, D. M. Oseid, G. L. Kimball, W. M. Koenst, and D. T. Lind. 1979. Acute and chronic toxicity of HCN to fish and invertebrates, EPA-600/3-79-009. U.S. Environmental Protection Agency.

Smith, L. R., T. M. Holsen, N. C. Ibay, R. M. Block, and A. B. de Leon. 1985. Studies on the acute toxicity of fluoride ion to stickleback, fathead minnow, and rainbow trout. Chemosphere 14(9):1383-1389.

Snarski, V. M., and G. F. Olson, 1982. Chronic toxicity and bioaccumulation of mercuric chloride in the fathead minnow (Pimephales promelas). Aquat. Toxicol. 2:143-156.

Spehar, R. L. 1976. Cadmium and zinc toxicity to tlagfish, Jordanella floridae. J. Fish. Res. Board Can. 33:1939-1945.

Spehar, R. L., J. T. Fiandt, R. L. Anderson, and D. L. DeFoe. 1980. Comparative toxicity of arsenic compounds and their accumulation in invertebrates and fish. Arch. Environ. Contam. Toxicol. 9:53-63.

Spehar, R. L., H. P. Nelson, M. J. Swanson, and J. W. Renoos. 1985. Pentachlorophenol toxicity to amphipods and fathead minnows at different $\mathrm{pH}$ values. Environ. Toxicol. Chem. 4:389--397.

Spehar, R. L., D. K. Tanner, and J. H. Gibson. 1982. Effects of kelthane and pydrin on early life stages of fathead minnows (Pimephales promelas) and amphipods (Hyalella azteca). IN J. G. Pearson, R. B. Foster, and W. E. Bishop (eds.), Aquatic Toxicology and Hazard Assessment, Fifth Conference, ASTM STP766. American Society for Testing and Materials, Philadelphia, Penn.

Spehar, R. L., D. K. Tanner, and B. R. Nordling. 1983. Toxicity of the synthetic pyrethroids, permethrin, and $\mathrm{AC} 222,705$ and their accumulation in early life stages of fathead minnows and snails. Aquat. Toxicol. 3:171-182.

Stephan, C. E., D. I. Mount, D. J. Hansen, J. H. Gentile, G. A. Chapman, and W. A. Brungs. 1985. Guidelines for deriving numerical national water quality criteria for the protection of aquatic organisms and their uses. PB85-227()49. National Technical Information Service, Springfield, Va. 
Stephan, C. E., and J. R. Rogers. 1985. Advantages of using regression analysis to calculate results of chronic toxicity tests. pp. 328-339. IN R. C. Bahner and D. J. Hansen (eds.), Aquatic Toxicology and Hazard Assessment: Eighth Symposium, American Society for Testing and Materials, Philadelphia.

Stephan, E. E. and R. J. Erickson. n.d. Guidelines for deriving an aquatic life pesticide concentration. U.S. Environmental Protection Agency, Environmental Research Laboratory, Duluth, MN, unpublished document.

Stevens, D. G., and G. A. Chapman. 1984. Toxicity of trivalent chromium to early life stages of steelhead trout. Environ. Toxicol. Chem. 3:125-133.

Suter, G. W., II. 1989. Ecological endpoints. pp. 2-1-2-28. IN W. Warren-Hicks, B. R. Parkhurst, and S. S. Baker, Jr. (eds.), Ecological Assessment of Harardous Waste Sites: A Field and Laboratory Reference Document. EPA 600/3-89/013. Corvallis Environmental Research Laboratory, Oregon.

Suter, G. W., II, A. E. Rosen, E. Linder, and D. F. Parkhurst. 1987. End points for responses of fish to chronic toxic exposures. Environ. Toxicol. Chem. 6:793-809.

Suter, G. W., II. 1992. Ecological Risk Assessment. Lewis Publishers, Chelsea, MI.

Suter, G. W., II, A. Redfearn, R. K. White, and R. A. Shaw. 1992. Approach and strategy for performing ecological risk assessments for the Department of Energy Oak Ridge Field Office Environmental Restoration Program. ES/ER/TM-33. Environmental Restoration Division, Oak Ridge National Laboratory, Oak Ridge, TN.

Thompson, R. S., and N. G. Carmichael. 1989. 1,1,1-Trichloroethane: medium-term toxicity to carp, daphnids, and higher plants. Ecotox. Environ. Safety 17:172-182.

Thurston, R.V., R.C. Russo, E.L. Meyn, and R.K. Zajdel. 1986. Chronic toxicity of ammonia to fathead minnows. Trans. Amer. Fish. Soc. 115:196-207.

Van der Schalie, W. H. 1983. The acute and chronic toxicity of 3,5-dinitroaniline, 1,3-dinitrobenzene, and 1,3,5-trinitrobenzene to freshwater aquatic organisms. Technical Report 8305. U.S. Army Medical Bioengineering Research and Development Laboratory, Fort Detrick, Frederick, MD.

Van Leeuwen, C. J., and H. Maas. 1985. The aquatic toxicity of 2,6-dichlorobenzamide (BAM), a degradation product of the herbicide dichlobenil. Environ. Pollut. Ser. A. 37:105-115.

Ward, T. J., and R. L. Boeri. 1991a. Early Life Stage Toxicity of Di-n-Butyl Phthalate (DnBP) to the Rainbow Trout (Oncorhynchus mykiss) Under Flow-Through Conditions. 9102-CMA. Chemical Manufacturers Association, Washington, D.C.

Ward, T. J., and R. L. Boeri. 1991b. Early Life Stage Toxicity of Dimethyl Phthalate (DMP) to the Rainbow Trout (Oncorhynchus mykiss) Under Flow-Through Conditions. 9101-CMA. Chemical Manufacturers Association, Washington, D.C. 
Appendix A

DATA USED FOR ADVISORY VALUE CALCULATIONS 
Table A-1. Data used for advisory value calculations (all valucs in micrograms per liter)

\begin{tabular}{llll}
\hline Chemical name/data type & Value & Species & Source \\
\hline Inorganics &
\end{tabular}

\section{Antimony}

Acute values

$\begin{aligned} 9,000 & \text { Daphnia magna } \\ 18,800 & \text { Daphnia magna } \\ 21,900 & \text { Fathead minnow }\end{aligned}$

EPA 1980a

18,800

Fathead minnow

ibid.

ihid.

Pesticide Acute Value

220

Acute-Chronic ratios

3.481

Daphnia magna

13.69

Fathead minnow

Kimball n.d.

Pesticide $\mathrm{A}-\mathrm{C}$ ratio

12.4

PAV / PACR

18

\section{Arsenic V}

Acute values

$$
\begin{array}{r}
<8,100 \\
7,400 \\
3,600 \\
49,600 \\
850 \\
10,800 \\
25,600 \\
49,000
\end{array}
$$

Daphnia magna
Daphnia magna
Daphnia pulex
Daphnia pulex
Bosmina longirostris
Rainbow trout
Fathead minnow
Mosquitofish

Epa 1985a

ibid.

ibid.

ibid.

ibid.

ibid.

ibid.

ibid.

PAV

27

Acute-Chronic ratios

28.7

Fathead minnow

DeFoe 1982

PACR

35.8

PAV / PACR

0.77

\section{Barium}

Acute values

410,000

Daphnia magna

(AQUIRE)*

PAV

4,362

PACR

40

PAV / PACR

110 


$$
\text { A-4 }
$$

Table A-1 (continued)

\begin{tabular}{llll}
\hline Chemical name/data type & Value & Species & Source \\
\hline Inorganics (continued)
\end{tabular}

Beryllium

Acute values

\begin{tabular}{|c|c|c|}
\hline 7,900 & Daphnia magna & EPA 1980e \\
\hline 2,500 & Daphnia magna & ibid. \\
\hline 4,800 & Goldfish & ibid. \\
\hline 3,250 & Fathead minnow & ibid. \\
\hline 200 & Fathead minnow & ibid. \\
\hline 150 & Fathead minnow & ibid. \\
\hline 150 & Fathead minnow & ibid. \\
\hline 11,000 & Fathead minnow & ibid. \\
\hline 20,000 & Fathead minnow & ibid. \\
\hline 15,000 & Fathead minnow & ibid. \\
\hline 18,000 & Fathead minnow & ibid. \\
\hline 4,400 & Flagfish & ibid. \\
\hline 3,530 & Flagfish & ibid. \\
\hline $\mathbf{3 , 5 3 0}$ & Flagfish & ihid. \\
\hline 32,000 & Guppy & ibid. \\
\hline 28,000 & Guppy & ibid. \\
\hline 32,000 & Guppy & ibid. \\
\hline 24,000 & Guppy & ibid. \\
\hline 160 & Guppy & ibid. \\
\hline 19,000 & Guppy & ibid. \\
\hline 450 & Guppy & ibid. \\
\hline 130 & Guppy & ibid. \\
\hline 200 & Guppy & ibid. \\
\hline 20,000 & Guppy & ibid. \\
\hline 13,700 & Guppy & ihid. \\
\hline 6,100 & Guppy & inid. \\
\hline 160 & Guppy & inid. \\
\hline 12,000 & Bluegill & ibid. \\
\hline 1,300 & Bluegill & ibid. \\
\hline \multicolumn{3}{|l|}{55} \\
\hline 472 & Daphnia magna & Kimball n.d \\
\hline \multicolumn{3}{|l|}{91.1} \\
\hline 0.61 & & \\
\hline
\end{tabular}

PAV / PACR

0.6

\section{Cobalt}

Acute values

$5,(9 \times x)$

Daphnia magna

Kimball n.d.

inid. 


$$
\text { A-5 }
$$

Table A-1 (continued)

\begin{tabular}{|c|c|c|c|}
\hline Chemical name/data type & Value & Species & Source \\
\hline \multicolumn{4}{|c|}{ Inorganics (continued) } \\
\hline Acute values (cont.) & 531 & Fathead minnow & Lind et al. 1978 \\
\hline PAV & 24 & & \\
\hline Acute-Chronic ratios & $\begin{array}{r}1,175 \\
290\end{array}$ & $\frac{\text { Daphnia magna }}{\text { Fathead minnow }}$ & $\begin{array}{l}\text { Kimball n.d. } \\
\text { Kimball n.d. }\end{array}$ \\
\hline PACR & 83.6 & & \\
\hline PAV / PACR & 0.29 & & \\
\hline \multicolumn{4}{|l|}{ Fluoride } \\
\hline Acute values & $\begin{array}{r}98,000 \\
251,000 \\
390,247 \\
200,000 \\
245,987 \\
125,000\end{array}$ & $\begin{array}{l}\text { Daphnia magna } \\
\text { Daphnia magna } \\
\text { Stickleback } \\
\text { Rainbow trout } \\
\text { Fathead minnow } \\
\text { Brown trout }\end{array}$ & $\begin{array}{l}\text { Dave 1984b } \\
\text { Fieser et al. } 1986 \\
\text { Smith et al. } 1985 \\
\frac{\text { ibid. }}{\text { ibid. }} \\
\text { (AQUIRE)* }\end{array}$ \\
\hline PAV & 3,734 & & \\
\hline Acute-Chronic ratios & $\begin{array}{r}8.33 \\
22.27\end{array}$ & $\begin{array}{l}\text { Daphnia magna } \\
\text { Daphnia magna }\end{array}$ & $\begin{array}{l}\text { Fieser et al. } 1986 \\
\text { Dave } 1984 b\end{array}$ \\
\hline PACR & 19.5 & & \\
\hline PAV / PACR & 191 & & \\
\hline \multicolumn{4}{|l|}{ Magnesium } \\
\hline Acute values & 64,700 & Scud & (AQUIRE)* \\
\hline PAV & 6.5 & & \\
\hline PACR & 40 & & \\
\hline PAV / PACR & 0.16 & & \\
\hline \multicolumn{4}{|l|}{ Manganese } \\
\hline Acute values & $\begin{array}{l}19,400 \\
33,800\end{array}$ & $\frac{\text { Daphnia magna }}{\text { Fathead minnow }}$ & $\begin{array}{l}\text { Kimball n.d. } \\
\text { ibid. }\end{array}$ \\
\hline
\end{tabular}


A-6

Table A-1 (continued)

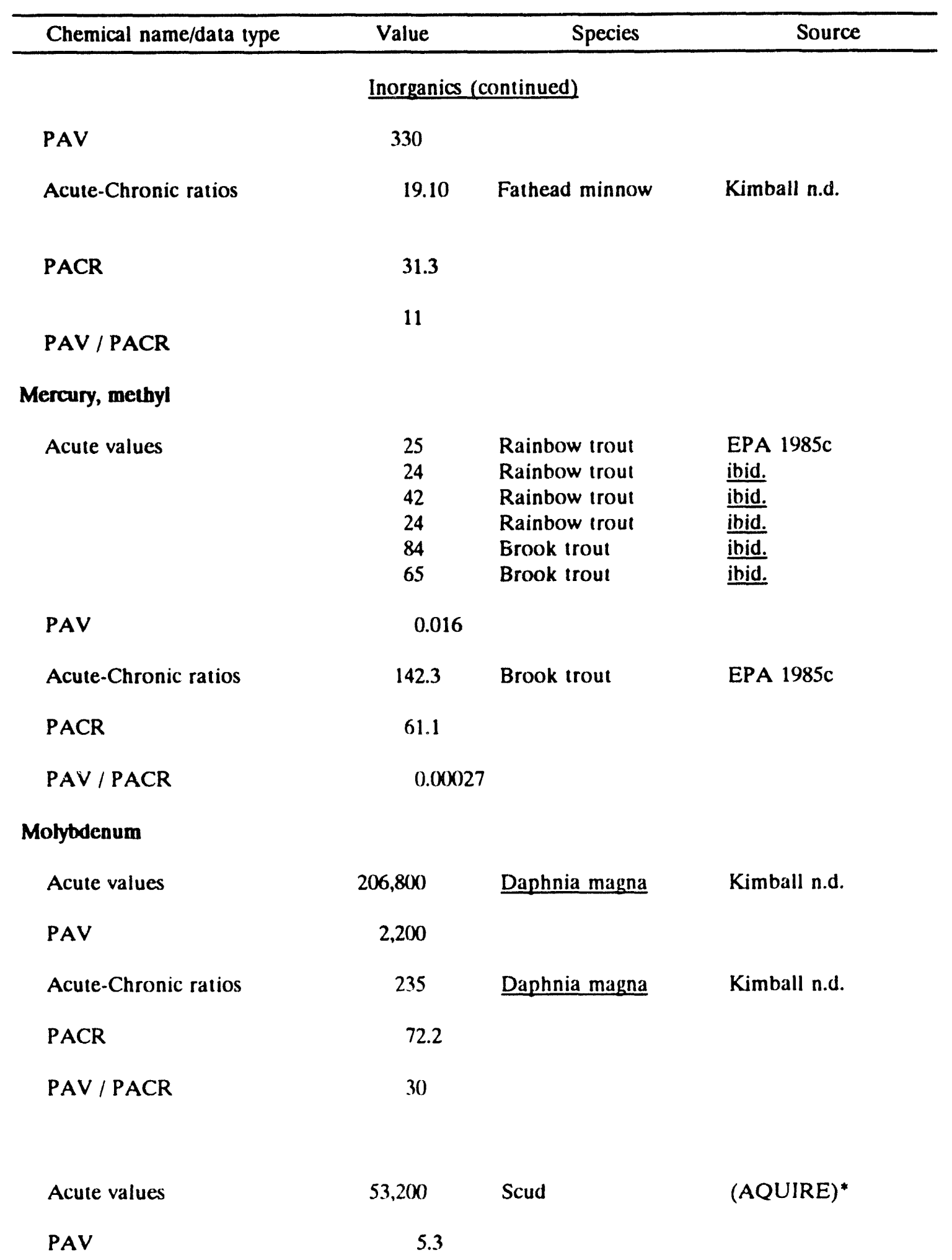


A-7

Table A-1 (continued)

\begin{tabular}{lcc}
\hline Chemical name/data type & Value & Species \\
& $\frac{\text { Inorganics (continued) }}{2}$ \\
PACR & 40 \\
PAV / PACR & 0.13
\end{tabular}

\section{Silver}

Final Acute Value

4.1

EPA 1980u

Acute-Chronic ratios

2.0

54

EPA 1980u

ihid.

PACR

65.1

FAV / PACR

$$
0.063
$$

\section{Sodium}

Acute values

$1,820,000$

Daphnia magna

(AQUIRE)*

PAV

19,000

PACR

40

PAV / PACR

480

Thallium

Acute values

$$
\begin{array}{r}
2,180 \\
910 \\
1,800 \\
132,000 \\
121,000
\end{array}
$$

PAV

27

Acute-Chronic ratios

7.0 Daphnia magna

31.58

Daphnia magna

Daphnia magna

Fathead minnow

Bluegill

Bluegill

EPA $1980 \mathrm{x}$

inid.

ihid.

ihid.

ihid.

\section{PACR}

10.8

PAV / PACR

2.5

\section{Uranium}

Acute values

Kimball n.d.

ibid. 
A-8

Table A-1 (continued)

\begin{tabular}{lcll}
\hline Chemical name/data type & Value & Species & Source \\
\hline & \multicolumn{2}{c}{ Inorganics (continued) } & \\
\cline { 2 - 3 } & 3,100 & Fathead minnow & $\frac{\text { ibid. }}{\text { ibid. }}$ \\
Acute values (cont.) & 3,700 & Fathead minnow & Fathead minnow \\
PAV & 135,000 & ibid. \\
PACR & 0.28 & \\
PAV / PACR & 40 &
\end{tabular}

Vanadium

Acute values

1,520

1,850

7,000

11,200

4,060

PAV

Acute-Chronic ratios

Final A-C ratio

PAV / FACR

\section{Zirconium}

Acute values

14,000

18,000

115,000

240,000

15,000

270,000
Daphnia magna

Fathead minnow

Brook trout

Flagfish

Daphnia magna

44

10.88 Fathead minnow

$140 \quad$ Flagfish

2.137 Daphnia magna

14.8

2.97
Kimball n.d.

ibid.

Ernst and Garside 1986

Holdway and Sprague 1979

Beusen and Neven 1987

Kimball n.d.

Holdway and Sprague 1979

Beusen and Neven 1987
Cushman et al. 1977

ibid.

ihid.

ihid.

ibid.

ihid. 
Table A-1 (continucd)

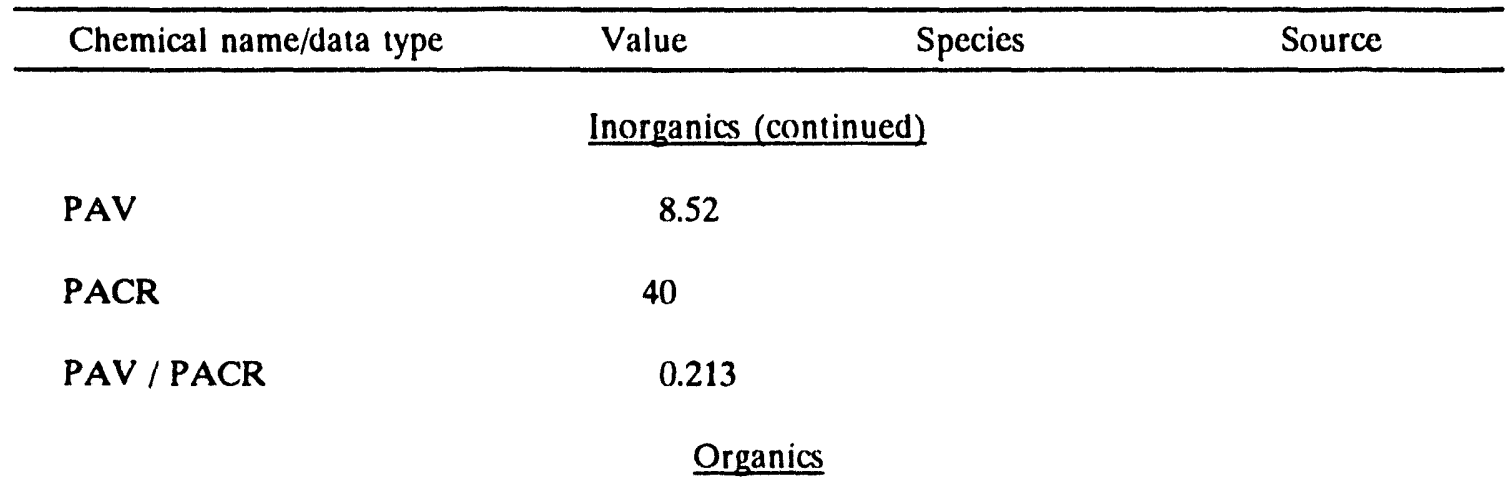

Acenaphthene

Acute values

41,200

1,700

Daphnia magna

Bluegill

EPA 1980a

ibid.

29

Acute-Chronic ratios

PACR

PAV / PACR

2.2

Acetone

Acute values

$$
\begin{array}{r}
8,300,000 \\
8,300,000 \\
1,300,000 \\
8,140,000 \\
7,310,000) \\
5,540,000 \\
13,500,000 \\
12,600,000 \\
13,300,000 \\
12,100,000 \\
8,800,000 \\
7,460,000 \\
7,810,000
\end{array}
$$

1.472

Fathead minnow

Cairns and Nebeker 1982

(AQUIRE)*

ibid.

ihid.

ihid.

Mosquitofish

Fathead minnow

Fathead minnow

Rainbow trout

Daphnia magna

Daphnia magna

Daphnia magna

Daphnia magna

Daphnia pulex

Daphnia cucullata

Daphnia cucullata ihid.

ihid.

ibid.

inid.

ihid.

ibid.

ibid.

ibid.

inid.

PAV

31,000

PACR

40)

PAV / PACR 


\section{A-10}

Table A-1 (continued)

\begin{tabular}{llll}
\hline Chemical name/data type & Value & Species & Source \\
\hline & Organics (continued)
\end{tabular}

Anthracene

Acute values

PAV

PACR

PAV / PACR

\section{Benze}

Acute values

PAV

PACR

PAV / PACR

\section{Benzidene}

Acute values

$$
\begin{aligned}
11.92 & \text { Sunfish } \\
2.78 & \text { Bluegill }
\end{aligned}
$$

0.00058

40

$$
0.00001
$$

4
Daphnia magna

Daphnia magna

Daphnia magna

Daphnia magna

Daphnia magna

Daphnia magna

Daphnia magna

Daphnia pulex

Daphnia pulex

Rainbow trout

Goldfish

Fathead minnow

Fathead minnow

Guppy

Mosquitofish

Bluegill
(AQUIRE)*

ibid.
EPA 1980c

ibid.

ibid.

ibid.

ibid.

ibid.

ibid.

ibid.

ibid.

ibid.

ibid.

ibid.

ibid.

ibid.

inid.

ibid.
126

40

3.15
7,400

4,350

2,500

16,200
Rainbow trout

Lake trout

Red shiner

Flagfish
EPA 1980b

ihid.

ibid.

ibid. 
A-11

Table A-1 (continucd)

\begin{tabular}{|c|c|c|c|}
\hline Chemical name/data type & Value & Species & Source \\
\hline \multicolumn{4}{|c|}{ Organics (continued) } \\
\hline PAV & 5.95 & & \\
\hline PACR & 40 & & \\
\hline PAV / PACR & 0.15 & & \\
\hline \multicolumn{4}{|l|}{ Benzo(a)anthracene } \\
\hline Acute values & $10^{*}$ & Daphnia pulex & (AQUIRE)* \\
\hline PAV & 0.11 & & \\
\hline PACR & 40 & & \\
\hline PAV / PACR & 0.0027 & & \\
\hline \multicolumn{4}{|l|}{ Benzo(a)pyrene } \\
\hline Acute values & $5^{*}$ & Daphnia pulex & (AQUIRE)* \\
\hline PAV & 0.053 & & \\
\hline PACR & 40 & & \\
\hline PAV / PACR & 0.0013 & & \\
\hline \multicolumn{4}{|l|}{ Benzoic acid } \\
\hline Acute values & 180,000 & Mosquitofish & $(A Q U I R E)^{*}$ \\
\hline PAV & 18 & & \\
\hline PACR & 40 & & \\
\hline PAV / PACR & 0.45 & & \\
\hline \multicolumn{4}{|l|}{ Benzylalcohol } \\
\hline Acute values & $\begin{array}{r}10,000 \\
460,000\end{array}$ & $\begin{array}{l}\text { Bluegill } \\
\text { Fathead minnow }\end{array}$ & $\begin{array}{l}\text { (AQUIRE)* } \\
\text { ibid. }\end{array}$ \\
\hline PAV & 5.88 & & \\
\hline PACR & 40 & & \\
\hline
\end{tabular}


A-12

Table A-1 (continued)

\begin{tabular}{|c|c|c|c|}
\hline Chemical name/data type & Value & Species & Source \\
\hline \multicolumn{4}{|c|}{ Organics (continued) } \\
\hline PAV / PACR & 0.147 & & \\
\hline \multicolumn{4}{|l|}{ BHC (other) } \\
\hline Acute values & $\begin{array}{r}1,000 \\
800\end{array}$ & $\begin{array}{l}\text { Daphnia magna } \\
\text { Daphnia magna }\end{array}$ & $\begin{array}{l}\text { (AQUIRE)* } \\
\text { ibid. }\end{array}$ \\
\hline PAV & 9.5 & & \\
\hline PACR & 40 & & \\
\hline PAV / PACR & 0.24 & & \\
\hline \multicolumn{4}{|l|}{ Bis(2-ethylhexyl)phthalate } \\
\hline Acute values & $\begin{array}{r}11,000 \\
133 \\
690 \\
6,180 \\
42,100 \\
32,900 \\
139,500 \\
149,200\end{array}$ & $\begin{array}{l}\text { Daphnia magna } \\
\text { Daphnia pulex } \\
\text { Channel catfish } \\
\text { Goldfish } \\
\text { Largemouth bass } \\
\text { Largemouth bass } \\
\text { Rainbow trout } \\
\text { Rainbow trout }\end{array}$ & $\begin{array}{l}\text { (AQUIRE)* } \\
\text { ibid. } \\
\frac{\text { ibid. }}{\text { ibid. }} \\
\frac{\text { ibid. }}{\text { ibid. }} \\
\frac{\text { ibid. }}{\text { ibid. }}\end{array}$ \\
\hline PAV & 3.17 & & \\
\hline Acute-Chronic ratios & $\begin{array}{r}38.73 \\
17,175\end{array}$ & $\frac{\text { Daphnia magna }}{\text { Rainbow trout }}$ & $\begin{array}{l}\text { Knowles et al. 1987† } \\
\text { Mehrle and Mayer } \\
1976 \dagger\end{array}$ \\
\hline PACR & 299 & & \\
\hline PAV / PACR & 0.0106 & & \\
\hline \multicolumn{4}{|l|}{ 2-Butanone } \\
\hline Acute values & $\begin{array}{l}5,600,000 \\
3,200,000 \\
5,091,000\end{array}$ & $\begin{array}{l}\text { Mosquitofish } \\
\text { Fathead minnow } \\
\text { Daphnia magna }\end{array}$ & $\begin{array}{l}\text { (AQUIRE)* } \\
\text { ibid. } \\
\text { ibid. }\end{array}$ \\
\hline PAV & 62,745 & & \\
\hline PACR & 40 & & \\
\hline PAV / PACR & 1,569 & & \\
\hline
\end{tabular}


A-13

Table A-1 (continued)

\begin{tabular}{llll}
\hline Chemical name/data type & Value & Species & Source \\
\hline & Organics (continued)
\end{tabular}

\section{Carbon disulfide}

Acute values

2,100

135,000

36

PAV

PACR

PAV / PACR

Carbon tetrachloride

Acute values

PAV

Acute-Chronic ratios

PACR

PAV / PACR

\section{Chlorobenzene}

Acute values

PAV

PACR

PAV / PACR

\section{Chloroform}

Acute values

35,200

43,100

125,000

27,300

690

63

86,000

51,620
0.8273

Fathead minnow

11.0

33,930

29,120

33,930

45,530

24,000

15,900

464

40

12

Daphnia magna

Fathead minnow

Blucgill

Bluegill

(AQUIRE)*

ihid.

Daphnia magna

Mosquitofish
EPA 1980f

ibid.

ibid.

ibid.

Kimball n.d.
Daphnia magna

Goldfish

Fathead minnow

Fathead minnow

Fathead minnow

Guppy

Bluegill

Bluegill
EPA $1980 \mathrm{~g}$

ihid.

inid.

inid.

inid.

ihid.

ibid.

ibid. 
A-14

Table A-1 (continucd)

\begin{tabular}{|c|c|c|c|}
\hline Chemical name/data type & Value & Species & Source \\
\hline \multicolumn{4}{|c|}{ Organics (continued) } \\
\hline Acute values (cont.) & $\begin{array}{r}66,800 \\
43,800 \\
115,000 \\
100,000\end{array}$ & $\begin{array}{l}\text { Rainbow trout } \\
\text { Rainbow trout } \\
\text { Bluegill } \\
\text { Bluegill }\end{array}$ & $\frac{\frac{\text { ibid. }}{\text { ibid. }}}{\text { ibid. }}$ \\
\hline PAV & 570 & & \\
\hline PACR & 40 & & \\
\hline PAV / PACR & 14 & & \\
\hline \multicolumn{4}{|l|}{ p,p'DDD } \\
\hline Acute values & $\begin{array}{c}2.5 \\
380 \\
380 \\
0.60 \\
0.64 \\
0.60 \\
0.86 \\
0.68 \\
2.4 \\
10 \\
16 \\
140 \\
740 \\
70 \\
4,400 \\
1,500 \\
42 \\
14 \\
42\end{array}$ & $\begin{array}{l}\text { Striped bass } \\
\text { Stonefly } \\
\text { Stonefly } \\
\text { Scud } \\
\text { Scud } \\
\text { Scud } \\
\text { Scud } \\
\text { Grass shrimp } \\
\text { Grass shrimp } \\
\text { Aquatic sowbug } \\
\text { Aquatic sowbug } \\
\text { Fowler's toad } \\
\text { Turbellarian } \\
\text { Rainbow trout } \\
\text { Fathead minnow } \\
\text { Channel catfish } \\
\text { Largemouth bass } \\
\text { Walleye } \\
\text { Bluegill }\end{array}$ & $\begin{array}{l}(\text { AQUIRE)* } \\
\text { ibid. } \\
\frac{\text { ibid. }}{\text { ibid. }} \\
\frac{\text { ibid. }}{\text { ibid. }} \\
\frac{\text { ibid. }}{\text { ibid. }} \\
\frac{\text { ibid. }}{\text { ibid. }} \\
\frac{\text { ibid. }}{\text { ibid. }} \\
\frac{\text { ibid. }}{\text { ibid. }} \\
\frac{\text { ibid. }}{\text { ibid. }} \\
\frac{\text { ihid. }}{\text { ihid. }} \\
\frac{\text { ihid. }}{\text { inis. }}\end{array}$ \\
\hline PAV & \multicolumn{2}{|c|}{0.017} & \\
\hline PACR & \multicolumn{2}{|l|}{40} & \\
\hline PAV / PACR & \multicolumn{2}{|c|}{0.00042} & \\
\hline \multicolumn{4}{|l|}{ Decane } \\
\hline Acute values & 48,000 & Daphnia magna & (AQUIRE)* \\
\hline PAV & 190 & & \\
\hline
\end{tabular}


A-15

Table A-1 (continued)

\begin{tabular}{llll}
\hline Chemical name/data type & Value & Specics & Source \\
\hline & Organics (continued)
\end{tabular}

PACR

40

PAV / PACR

4.8

\section{Di-n-butyl phthalatc}

Acute values

$\begin{aligned} 1,200 & \text { Bluegill } \\ 731 & \text { Bluegill } \\ 2,100 & \text { Bluegill } \\ 760 & \text { Bluegill } \\ 700 & \text { Bluegill } \\ 1,3(0) & \text { Fathead minnow } \\ 1,3(0) & \text { Fathead minnow } \\ 2,020 & \text { Fathead minnow } \\ 2,910 & \text { Channel catfish } \\ 2,910 & \text { Channel catfish } \\ 2,900 & \text { Channel catfish } \\ 6,470 & \text { Rainbow trout } \\ 6,470 & \text { Rainbow trout } \\ 2,600 & \text { Rainbow trout } \\ 2,100 & \text { Scud } \\ 10,000 & \text { Crayfish } \\ 3,700 & \text { Daphnia magna } \\ 5,200 & \text { Daphnia magna }\end{aligned}$

(AQUIRE)*

ihid.

inid.

ibid.

inid.

ihid.

ihid.

ibid.

ihid.

ihid.

ihid.

inid.

ibid.

ihid.

ihid.

ihid.

ihid.

ihid.

PAV

45

Acute-Chronic ratios

2.817 Fathead minnow

McCarthy and

Whitmore 1985

9.437

Daphnia magna

ihid.

PACR

10.2

PAV / PACR

4.4

Diebenzofuran

Acute values

$7,500)^{*}$

Daphnia magna

(AQUIRE)*

PAV

80

PACR

40)

PAV / PACR 


\section{A-16}

Table A-1 (continued)

\section{Chemical name/}

Acute values

$$
202,000
$$

Guppy

(AQUIRE)*

PAV

20

PACR

40

PAV / PACR

0.51

\section{1,2-Dichloroethane}

Acute values

270,000

218,000

118,000

550,000

431,000

PAV

Acute-Chronic ratios

PACR

PAV / PACR

\section{1,1-Dichlorocthene}

Acute values

$$
\begin{array}{r}
11,600 \\
79,000 \\
169,000 \\
108,000 \\
73,900
\end{array}
$$

PAV

PACR

PAV / PACR

\section{1,2-Dichloroethenc}

Acute values
2,314

5.9 Fathead minnow

17.76 Daphnia magna

16.1

144

Daphnia magna

Daphnia magna

Fathead minnow

Bluegill

Bluegill

Richter et al. 1983

EPA 1980h

ibid.

ibid.

ibid.

Ahmed et al. 1984

Richter et al. 1983
EPA 1980k

ibid.

inid.

ibid.

ibid. 
A-17

Table A-1 (continued)

\begin{tabular}{lccc}
\hline Chemical name/data type & Value & Species & Source \\
\hline & Organics (continued) & \\
PAV & 14 & \\
PACR & 40 & \\
PAV / PACR & 0.34 & \\
1,3-Dichloropropene (total) & & \\
Acute values & 6,150 & Daphnia magna & EPA 1980I \\
& 6,060 & Bluegill \\
PAV & 100 & \\
PACR & 40 & \\
PAV / PACR & 2.6 &
\end{tabular}

\section{Diethylphthalate}

Acute values

120,000

$52,000^{*}$

$75,000^{*}$

Bluegill

Daphnia magna

Daphnia magna

(AQUIRE)*

ibid.

ibid.

PAV

1,100

PACR

40

PAV / PACR

27

Ethylbenzene

Acute values

$\begin{array}{rll}75,000 & \text { Daphnia magna } & \text { EPA 1980m } \\ 94,440 & \text { Goldfish } & \text { ibid. } \\ 48,510 & \text { Fathead minnow } & \text { ibid. } \\ 42,330 & \text { Fathead minnow } & \text { ibid. } \\ 97,100 & \text { Guppy } & \text { ibid. } \\ 32,000 & \text { Bluegill } & \text { ibid. } \\ 155,000 & \text { Bluegill } & \text { ibid. } \\ 1,079 & & \\ 40 & & \\ & \end{array}$


A-18

Table A-1 (continuod)

\begin{tabular}{llll}
\hline Chemical name/data type & Value & Species & Source \\
\hline & Organics (continued)
\end{tabular}

Fluoranthene

Acute values

325,000

3,980

Daphnia magna

EPA 1980n

69

PAV

69

PACR

40

PAV / PACR

1.7

Hecane

Acute values

$150,000 \ddagger$

$4,480,000$ t

Golden orfe

Golden orfe

(AQUIRE)*

ibid.

PAV

82

PACR

40

PAV / PACR

2.1

\section{2-Hexanone}

Acute values

$428,000 \pm$

Fathead minnow

(AQUIRE)*

PAV

43

PACR

40)

PAV / PACR

1.1

1-Methylnaphthalene

Acute values

9,000

Fathead minnow

(AQUIRE)*

PAV

0.90

PACR

40

PAV / PACR

0.023

4-Methyl-2-pentanone

Acute values

$509,0 \times(x)$

Fathead minnow

(AQUIRE)* 
A-19

Table A-1 (continued)

\begin{tabular}{llll}
\hline Chemical name/data type & Value & Species & Source \\
\hline & Organics (continued)
\end{tabular}

PAV

Acute-Chronic ratios

PACR

PAV / PACR

\section{2-Methylphenol}

Acute values

PAV

PACR

PAV / PACR
51

608

Fathead minnow

Veith et al. 1983

22.2

2.3

12,550

13,420

18,200

20,780

23,250

8,400

17,400

15,500

9,800

8,600

23,800

23,100

15,100

15,800

15,800

5,000

14,000

10,800

8,500

Fathead minnow

Fathead minnow

Fathead minnow

Bluegill

Goldfish

Rainbow trout

Daphnia cucullata

Daphnia cucullata

Daphnia magna

Daphnia magna

Daphnia magna

Daphnia magna

Daphnia magna

Daphnia magna

Daphnia magna

Daphnia magna

Daphnia magna

Daphnia pulex

Daphnia pulex
(AQUIRE)*

ibid.

ibid.

ibid.

ibid.

ibid.

ibid.

ibid.

ibid.

ibid.

ibid.

ibid.

ibid.

ibid.

ibid.

ibid.

ibid.

ibid.

ihid.

310

40 
Table A-1 (continued)

\begin{tabular}{llll}
\hline Chemical name/data type & Value & Species & Source \\
\hline & Organics (continued) &
\end{tabular}

Mercury, methyl

Acute values

25
24
42
24
84
65

Rainbow trout

Rainbow trout

Rainbow trout

Rainbow trout

Brook trout

Brook trout

EPA 1985c

ibid.

ibid.

ibid.

ibid.

0.016

PAV

142.3

Brook trout

EPA $1985 c$

Acute-Chronic ratios

Methylene chloride

Acute values

220,000

Bluegill

Fathead minnow

Fathead minnow

502,000

Daphnia magna

(AQUIRE)*

ibid.

ihid.

220,000

4,314

PAV

4.65

Fathead minnow

Dill et al. 1987

Acute-Chronic ratios

PACR

PAV / PACR

\section{Naphthalene}

Acute values

4,900

8,900

150,000

PAV

55

Acute-Chronic ratios

10.65 Fathead minnow

DeGraeve et al. 1982

PACR

25.7

PAV / PACR

Daphnia magna

Rainbow trout

Fathead minnow

Fathead minnow

Mosquitofish

EPA $1980 q$

ihid.

ihid.

ibid.

ibid. 
Table A-1 (continued)

Chemical name/data type

Value

Species

Source

Organics (continued)

\section{4-Nitrophenol}

Acute values

PAV

Acute-Chronic ratios

PACR

PAV / PACR

\section{N-Nitrosodiphenylamine}

Acute values

PAV

PACR

PAV / PACR

\section{3-Octanone}

Acute values

PAV

PACR

PAV / PACR

$$
\begin{array}{r}
8,300 \\
59,000 \\
62,000 \\
41,000 \\
7,900 \\
15,000 \\
22,000 \\
8,400 \\
20,000 \\
8,400 \\
7,680 \\
13,200
\end{array}
$$

Bluegill

Fathead minnow

Fathead minnow

Fathead minnow

Rainbow trout

Channel catfish

Daphnia magna

Daphnia magna

Daphnia magna

Daphnia magna

Daphnia magna

River snail

255

2.83

Daphnia magna

16.5

15

5,800

7,800

Bluegill

Daphnia magna

100

40)

2.5

$80,000^{*}$

$517,000^{*}$

Goldfish

Daphnia magna
(AQUIRE)*

ihid.

ibid.

ibid.

ibid.

ibid.

ibid.

ibid.

ibid.

ibid.

ihid.

ihid.

Francis et al. 1986†
(AQUIRE)*

ibid.
(AQUIRE)*

ibid.
$: 0$

40

34 
A. 22

Table A-1 (continued)

\begin{tabular}{|c|c|c|c|}
\hline Chemical name/data type & Value & Species & Source \\
\hline & \multicolumn{3}{|c|}{ Organics (continued) } \\
\hline Acute values & $\begin{array}{l}1,200 \\
1,170 \\
1,170\end{array}$ & $\begin{array}{l}\text { Cutthroat trout } \\
\text { Cutthroat trout } \\
\text { Cutthroat trout }\end{array}$ & $\begin{array}{l}\text { (AQUIRE)* } \\
\text { ibid. } \\
\text { ibid. }\end{array}$ \\
\hline PAV & 0.12 & & \\
\hline PACR & 40 & & \\
\hline PAV / PACR & 0.0029 & & \\
\hline \multicolumn{4}{|l|}{ PCBs: Aroclor ${ }^{\bullet} 1232$} \\
\hline Acute values & $\begin{array}{l}2,500 \\
2,500 \\
2,500 \\
1,900\end{array}$ & $\begin{array}{l}\text { Cutthroat trout } \\
\text { Cutthroat trout } \\
\text { Cutthroat trout } \\
\text { Cutthroat trout }\end{array}$ & $\begin{array}{l}\text { (AQUIRE)* } \\
\frac{\text { ibid. }}{\text { ibid. }} \\
\text { ibid. }\end{array}$ \\
\hline PAV & 0.23 & & \\
\hline PACR & 40 & & \\
\hline PAV / PACR & 0.0058 & & \\
\hline \multicolumn{4}{|l|}{ PCBs: Aroclor ${ }^{\bullet} 1242$} \\
\hline Acute values & $\begin{array}{r}73 \\
10 \\
400 \\
15 \\
300\end{array}$ & $\begin{array}{l}\text { Scud } \\
\text { Scud } \\
\text { Damselfly } \\
\text { Fathead minnow } \\
\text { Fathead minnow }\end{array}$ & $\begin{array}{l}\text { EPA 1980t } \\
\frac{\text { ibid. }}{\text { ibid. }} \\
\text { ihid. } \\
\text { ihid. }\end{array}$ \\
\hline PAV & 0.21 & & \\
\hline Acute-Chronic ratios & $\begin{array}{c}14.90 \\
7.454\end{array}$ & $\begin{array}{l}\text { Scud } \\
\text { Fathead minnow }\end{array}$ & $\begin{array}{l}\text { EPA 1980t } \\
\text { ibid. }\end{array}$ \\
\hline PACR & 16.4 & & \\
\hline PAV / PACR & 0.01 & & \\
\hline PCBs: Aroclor ${ }^{\circledR} 1248$ & & & \\
\hline Acute values & 52 & Scud & EPA $1980 t$ \\
\hline
\end{tabular}


A-23

Table A-1 (continued)

\begin{tabular}{|c|c|c|c|}
\hline Chemical name/data type & Value & Species & Source \\
\hline \multicolumn{4}{|c|}{ Organics (continued) } \\
\hline Acute values (cont.) & 29 & Scud & ibid. \\
\hline PAV & 0.004 & & \\
\hline Acute-Chronic ratios & 8.788 & Scud & EPA $1980 t$ \\
\hline PACR & 24.1 & & \\
\hline PAV / PACR & 0.0002 & & \\
\hline \multicolumn{4}{|l|}{ PCBs: Aroclor 1254} \\
\hline Acute values & $\begin{array}{r}2,400 \\
200 \\
7.7\end{array}$ & $\begin{array}{l}\text { Scud } \\
\text { Damselfly } \\
\text { Fathead minnow }\end{array}$ & $\begin{array}{l}\text { EPA } 1980 t \\
\text { ihid. } \\
\text { ihid. }\end{array}$ \\
\hline PAV & 0.37 & & \\
\hline Acute-Chronic ratios & 2.655 & Fathead minnow & EPA 1980t \\
\hline PACR & 16.20 & & \\
\hline PAV / PACR & 0.02 & & \\
\hline \multicolumn{4}{|l|}{ PCBs: Aroclor 1260} \\
\hline Acute values & $\begin{array}{l}60,900 \\
61,000 \\
25,000\end{array}$ & $\begin{array}{l}\text { Cutthroat trout } \\
\text { Cutthroat trout } \\
\text { Cutthroat trout }\end{array}$ & $\begin{array}{l}\text { (AQUIRE)* } \\
\text { ibid. } \\
\text { ibid. }\end{array}$ \\
\hline PAV & 4.5 & & \\
\hline PACR & 40 & & \\
\hline PAV / PACR & 0.11 & & \\
\hline \multicolumn{4}{|l|}{ 1-Pentanol } \\
\hline Acute values & $\begin{array}{l}650,000 \\
400,000\end{array}$ & $\begin{array}{l}\text { Bluegill } \\
\text { Rainbow trout }\end{array}$ & $\begin{array}{l}\text { (AQUIRE)* } \\
\text { ibid. }\end{array}$ \\
\hline PAV & 240 & & \\
\hline PACR & 40 & & \\
\hline
\end{tabular}


Table A-1 (continucd)

\begin{tabular}{llll}
\hline Chemical name/data type & Value & Species & Source \\
\hline Organics (continued) &
\end{tabular}

PAV / PACR

Phenanthrene

Acute values

\begin{tabular}{|c|c|c|}
\hline 960 & Daphnia pulex & $(A Q U I R E)^{*}$ \\
\hline 490 & Midge & ibid. \\
\hline 700 & Daphnia magna & ibid. \\
\hline 843 & Daphnia magna & ibid. \\
\hline 734 & $\overline{\text { Daphnia magna }}$ & ibid. \\
\hline
\end{tabular}

PAV

8.4

Acute-Chronic ratios

4.8 Daphnia pulex

Geiger and Buikema 1982

PACR

19.8

PAV / PACR

0.43

Phenol

Acute values

$$
\begin{array}{r}
248,000 \\
94,000 \\
14,000 \\
36,400 \\
58,100 \\
57,000 \\
122,000 \\
108,000 \\
8,032 \\
44,500 \\
35,000 \\
16,700 \\
36,300 \\
26,000 \\
34,900 \\
16,400 \\
19,000
\end{array}
$$

\begin{tabular}{|c|c|}
\hline Rotifer & EPA 1980s \\
\hline Snail & ibid. \\
\hline Daphnia longispina & ibid. \\
\hline Daphnia magna & ibid. \\
\hline Daphnia pulex & ibid. \\
\hline Polyphemus pediculus & ihid. \\
\hline Cyclops vernalis & ibid. \\
\hline Mesocyclops leukarti & ihid. \\
\hline Rainbow trout & ibid. \\
\hline Goldfish & ihid. \\
\hline Fathead minnow & ibid. \\
\hline Channel catfish & ibid. \\
\hline Flagfish & ihid. \\
\hline Mosquitofish & ihid. \\
\hline Guppy & ibid. \\
\hline Bluegill & ihid. \\
\hline $\begin{array}{l}\text { Mozambique } \\
\text { mouthbrooder }\end{array}$ & ibid. \\
\hline
\end{tabular}

PAV

448

\footnotetext{
a Because the list of acute values for phenol is so long, the species mean acute values have been substituted here.
} 
A-25

Table A-1 (continued)

\begin{tabular}{lccc}
\hline Chemical name/data type & Value & Species & Source \\
& \multicolumn{2}{c}{ Organics (continued) } & \\
\cline { 2 - 4 } & 14.06 & $\begin{array}{l}\text { Fathead minnow } \\
\text { Fathead minnow }\end{array}$ & $\begin{array}{l}\text { EPA 1980st } \\
\text { DeGracve et }\end{array}$ \\
Acute-Chronic ratios & 18.19 & & \\
PACR & 21.7 & & \\
PAV / PACR & 21 & & Fathead minnow \\
2-Propanol & & (AQUIRE)* \\
Acute values & & Fathead minnow & ibid. \\
& $11,130,000$ & ibid.
\end{tabular}

PAV 100

$\begin{array}{ll}\text { PACR } & 40\end{array}$

PAV / PACR $\quad 2.6$

\section{1,1,2,2-Tetrachloroethane}

Acute values

$\begin{array}{rll}21,000 & \text { Bluegill } & \text { (AQUIRE)* }^{*} \\ 20,300 & \text { Fathead minnow } & \text { ibid. } \\ 20,400 & \text { Fathead minnow } & \text { ibid. } \\ 62,000 & \text { Daphnia magna } & \text { ibid. } \\ 9,320 & \text { Daphnia magna } & \text { EPA 1980h } \\ 20,300 & \text { Fathead minnow } & \text { ibid. } \\ 21,300 & \text { Bluegill } & \text { ibid. }\end{array}$

PAV 399

Acute-Chronic ratios

8.46 Fathead minnow

6.289 Daphnia magna

Ahmed et al. 1984

Richter et al. 1983

PACR

12.9

PAV / PACR

\section{Tetrachlorocthene}

Acute values

Daphnia magna

Richter et al. 1983

Daphnia magna

Tanytarsus dissimilis

EPA 1980v

Rainbow trout

ihid.

Rainbow trout

ihid.

ibid. 
A-26

Table A-1 (continued)

\begin{tabular}{lcll}
\hline Chemical name/data type & Value & Species & Source \\
\hline & \multicolumn{2}{c}{ Organics (continued) } & \\
Acute values (cont.) & 13,460 & Fathead minnow & $\frac{\text { ibid. }}{\text { ibid. }}$ \\
& 18,400 & Fathead minnow & $\frac{\text { ibid. }}{\text { ibid. }}$ \\
& 21,400 & Fathead minnow &
\end{tabular}

Acute-Chronic ratios

16.02 Fathead minnow

Ahmed et al. 1984

11.35

Daphnia magna

Richter et al. 1983

PACR

19.4

PAV / PACR

8.8

Toluene

Acute values

60,000

313,000

22,800

57,680

34,270

42,330

59,300

24,000

17,500

Daphnia magna

Daphnia magna

Goldfish

Goldfish

Fathead minnow

Fathead minnow

Guppy

Bluegill

Bluegill

EPA 1980y

ibid.

ibid.

ibid.

ibid.

ibid.

ibid.

ibid.

ibid.

417

40

PACR

10.4

PAV / PACR

\section{1,1,1-Trichloroethane}

Acute values

$$
40,000
$$

52,800

105,000

Bluegill

Fathead minnow

Fathead minnow

(AQUIRE)*

ibid.

inid.

PAV

Acute-Chronic ratios

24

3.057 b Daphnia magna

Thompson \&

Carmichael 1989

\footnotetext{
based on a 17-d

$\mathrm{LC}_{50}$ (standard is $48 \mathrm{~h}$ ).
} 
A-27

Table A-1 (continued)

\begin{tabular}{llll}
\hline Chemical name/data type & Value & Species & Source \\
\hline & Organics (continued)
\end{tabular}

PACR

17.0

PAV / PACR

1.386

\section{1,1,2-Trichloroethane}

Acute values

81,700
18,000
43,000
190,000
170,000
170,000

PAV

Acute-Chronic ratios

PACR

PAV / PACR

\section{Trichloroethene}

Acute values

PAV

Acute-Chronic ratios

PACR

PAV / PACR

877

55
1,400

8.691 Fathead minnow 9.776

Daphnia magna

15.0

92

Fathead minnow Daphnia magna

Daphnia magna

Daphnia magna

Daphnia magna

Great pond snail

(AQUIRE)*

ibid.

ibid.

ibid.

ihid.

ibid.

Ahmed et al. 1984

Richter et al. 1983

$\begin{array}{rll}85,200 & \text { Daphnia magna } & \text { EPA } 1980 \mathrm{z} \\ 100,000 & \text { Daphnia magna } & \text { ibid. } \\ 94,000 & \frac{\text { Daphnia magna }}{\text { Daphnia magna }} & \frac{\text { ibid. }}{\text { ihid. }} \\ 41,000 & \text { Daphnia magna } & \frac{\text { ihid. }}{\text { ibid. }} \\ 43,000 & \frac{\text { Daphnia magna }}{\text { Daphnia magna }} & \frac{\text { ihid. }}{\text { ihid. }} \\ 55,000 & \text { Daphnia pulex } & \frac{\text { Daphnia pulex }}{\text { ibid. }} \\ 56,000 & \text { Fathead minnow } & \frac{\text { ibid. }}{\text { ibid. }} \\ 39,000 & \text { Fathead minnow } & \frac{\text { ibid. }}{\text { Smith ct al. } 1991} \\ 40,700 & \text { Bluegill } & \\ 66,800 & \text { Flagfish } & \end{array}$

$2.558 \quad$ Flagfish

Smith et al. 1991

16.0 
Table A-1 (continued)

\begin{tabular}{|c|c|c|c|}
\hline Chemical name/data type & Value & Species & Source \\
\hline
\end{tabular}

Vinyl acetate

Acute values

$\begin{aligned} 13,465 \mathrm{c} & \text { Fathead minnow } \\ 18,000 & \text { Bluegill } \\ 42,330 & \text { Goldfish } \\ 31,080 & \text { Guppy }\end{aligned}$

(AQUIRE)*

ibid.

ibid.

ihid.

PAV

32

PACR

40

PAV / PACR

0.80

\section{Vinyl chloride}

Acute values

PAV

PACR

PAV / PACR

\section{Xylene}

Acute values

780,000

99,500

59

PAV

PACR

PAV / PACR
$356,000 \ddagger$ $406,000 \pm$

38

40

0.95

Golden orfe

Golden orfe

(AQUIRE)*

ibid.
(AQUIRE)*

ibid.

\section{Common carp \\ Calanoid copepod}

$\underline{\mathrm{C}}$ Water hardness was varied in this test; a logarithmic regression was performed according to the National Guidelines, and $13,465 \mu \mathrm{g} / \mathrm{L}$ is the intercept value.

*These acute tests were of a non-standard duration. Standard durations are $48 \mathrm{~h}$. for daphnids and midges, and $96 \mathrm{~h}$. for all other aquatic animals.

tThese reports contained no acute value to accompany the chronic values, so a species mean acute value derived from other available studies was used to calculate the ratio.

\$These test results are unreliable in that either a test duration was not specified or the experimental methods were not usual or not consistent, but they are the best data available. 
Appendix B

FACTORS FOR ESTIMATION OF THE PESTICIDE ACUTE VALUE 
Table B-1. Factors for estimation of the PAV (Erickson and Stephan n.d.)

\begin{tabular}{|c|c|c|}
\hline $\begin{array}{l}\text { Number of } \\
\text { LC }_{50^{5}} \mathrm{a}\end{array}$ & $\begin{array}{l}\text { Factor for data sets } \\
\text { that include a } \\
\text { LC }_{50} \text { a } \\
\text { for a daphnid } \underline{\text { a }}\end{array}$ & 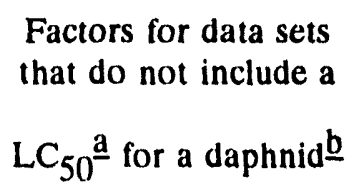 \\
\hline 1 & 94 & 10,000 \\
\hline 2 & 58 & 1,700 \\
\hline 3 & 51 & 420 \\
\hline 4 & 42 & 180 \\
\hline 5 & 31 & 110 \\
\hline 6 & 22 & 64 \\
\hline 7 & 13 & 40 \\
\hline
\end{tabular}

all $\mathrm{LC}_{50}$ should be from different families and otherwise should meet the requirements of data used to calculate final acute values (Stephan et al. 1985, EPA 1985a). Multiple LC $_{50}$ s for a species should be geometrically averaged to generate a species mean $\mathrm{LC}_{50}$. Species mean $\mathrm{LC}_{50}$ s for congeneric species should be geometrically averaged and those genus mean $\mathrm{LC}_{50}$ s should be used in the calculations.

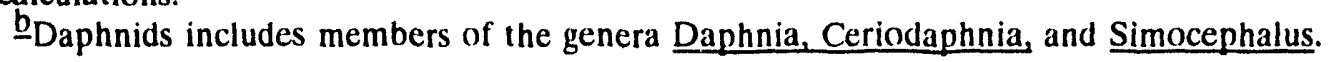




\section{DISTRIBUTION}

1. L. D. Bates

2. D. T. Bell

3. J. B. Cannon

4. R. B. Cook

5. J. H. Cushman

6. M. F. P. DeLozier

7. D. E. Fowler

8-9. M. A. Futrell

10. C. W. Gehrs

11. C. D. Goins

12. P. J. Halsey

13. S. G. Hildebrand

14. P. Kanciruk

15-16. G. A. Kerchner

17. B. L. Kimmel

18. V. Legg

19-21. D. M. Matteo

22-23. P. T. Owen
24. D. E. Reichle

25. G. E. Rymer

26. F. E. Sharples

27. D. S. Shriner

28. S. H. Stow

29-33. G. W. Suter II

34. K. B. Swain

35. R. I. Van Hook

36. D. R. Watkins

37. R. K. White

38. P. A. Schrandt

39. Central Research Library

40-44. ESD Library

45-49. ER Document Management Center

50-51. Laboratory Records Dept.

52. ORNL Patent Section

54. Office of Assistant Manager for Energy Research and Development, DOE Oak Ridge Field Office, P.O. Box 2001, Oak Ridge, TN 37831-8600

55. J. F. Franklin, Bloedel Professor of Ecosystem Analysis, College of Forest Resources, University of Washington, Anderson Hall AR-10, Seattle, WA 98195

56. R. C. Harriss, Institute for the Study of Earth, Oceans, and Space, Science and Engineering Research Building, University of New Hampshire, Durham, NH 03824

57. G. Y. Jordy, Director, Office of Program Analysis, Office of Energy Research, ER-30, G-226, U.S. Department of Energy, Washington, DC 20545

58-59. R. L. Nace, Branch Chief, Nonenrichment Facilities, Oak Ridge Program Division, Office of Eastern Area Programs, Office of Environmental Restoration, EM-423, Trevion 2, U.S. Department of Energy, Washington, DC 20585

60. R. H. Olsen, Professor, Microbiology and Immunology Department, University of Michigan, Medical Sciences II, \#5605, 1301 East Catherine Street, Ann Arbor, MI 48109-0620

61. A. Patrinos, Acting Director, Environmental Sciences Division, Office of Health and Environmental Research, ER-74, U.S. Department of Energy, Washington, DC 20585

62-63. R. C. Sleeman, DOE Oak Ridge Field Office, P.O. Box 2001, Oak Ridge, TN 378318540

64-65. J. T. Sweeney, DOE Cak Ridge Field Office, P.O. Box 2001, Oak Ridge, TN 378318541

66. D. W. Swindle, Radian Corporation, 120 South Jefferson Circle, Oak Ridge, TN 37830 
67-68. H. M. Thron, Chief, Enrichment Facilities, Oak Ridge Program Division, Office of Eastern Area Programs, Office of Environmental Restoration, EM-423, Trevion 2, U. S. Department of Energy, Washington, DC 20585.

69. F. J. Wobber, Environmental Sciences Division, Office of Health and Environmental Research, ER-74, U.S. Department of Energy, Washington, DC 20585

70-71. Office of Scientific and Technical Information, P.O. Box 62, Oak Ridge, TN 37831 

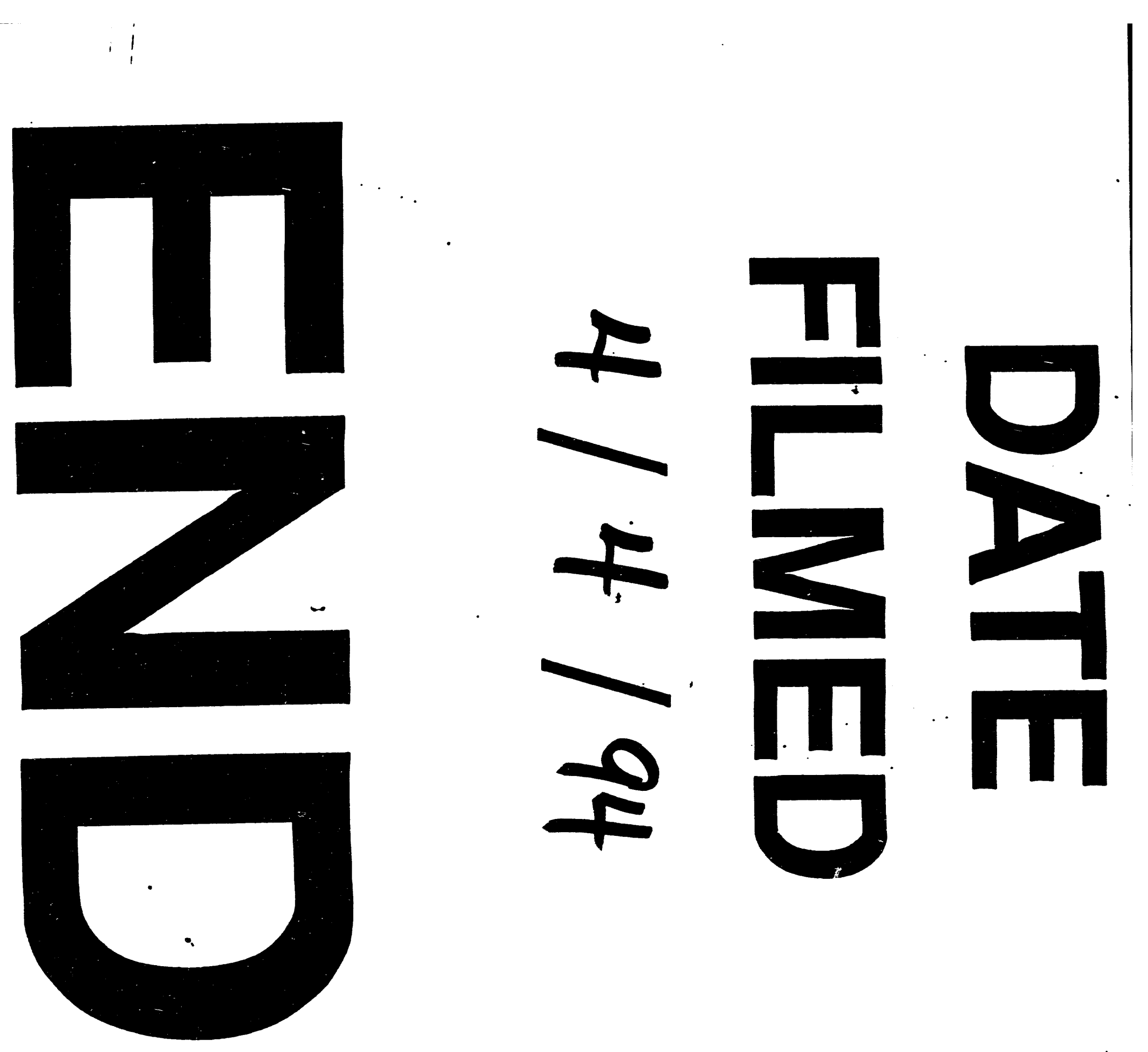